Supporting Information

\title{
Total Synthesis of (-)-L-755,807: Establishment of Relative and Absolute Configurations
}

\author{
Kosaku Tanaka III, Kenichi Kobayashi,* and Hiroshi Kogen*
}




\section{Table of Contents}

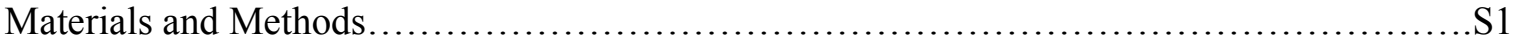

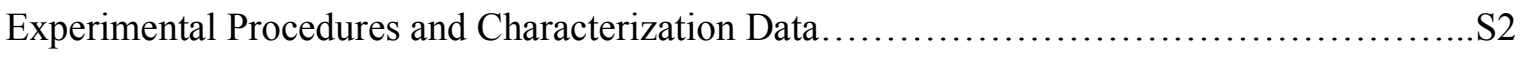

Experimental Spectra........................................................ 14

Materials and Methods: ${ }^{1} \mathrm{H}$ NMR spectra were measured on a JEOL JNM-AL300 (300 MHz) or a JEOL JNM-AL400 (400 MHz) instrument. The chemical shifts are expressed in ppm downfield from tetramethylsilane $(\delta=0)$ as an internal standard $\left(\mathrm{CDCl}_{3}\right.$ or $\mathrm{CD}_{2} \mathrm{Cl}_{2}$ solution). Splitting patterns are indicated as follows: $\mathrm{s}$, singlet; $\mathrm{d}$, doublet; $\mathrm{t}$, triplet; $\mathrm{q}$, quartet; $\mathrm{m}$, multiplet; br, broad peak. ${ }^{13} \mathrm{C}$ NMR spectra were measured at $100 \mathrm{MHz}$. The chemical shifts are reported in ppm, relative to the central line of a triplet at $77.0 \mathrm{ppm}$ for $\mathrm{CDCl}_{3}$ or a quintet at $53.8 \mathrm{ppm}$ for $\mathrm{CD}_{2} \mathrm{Cl}_{2}$. Infrared spectra (IR) were measured on a JASCO VALOR-III and were reported in wavenumbers $\left(\mathrm{cm}^{-1}\right)$. High resolution mass spectra (HRMS) were obtained using a JEOL JMS 700 instrument with a direct inlet system. Optical rotations were measured on a Jasco P-2200 polarimeter using a $100 \mathrm{~mm}$ pathlength cell. Melting points (m.p.) were measured on a Yanaco Micro Melting Point. Column chromatography was carried out on silica gel (40-100 mesh). Analytical thin-layer chromatography (TLC) was performed using $0.25 \mathrm{~mm}$ silica gel 60-F plates. 


\section{Experimental Procedures and Characterization Data}

\section{Di-tert-butyl}

(S)-3-((R)-1-((tert-butyldiphenylsilyl)oxy)-2-methylpropyl)oxirane-2,2-dicarboxylate (12)

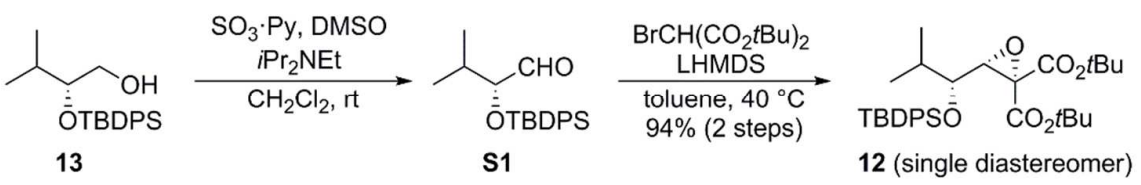

To a stirred solution of alcohol $13(2.01 \mathrm{~g}, 5.87 \mathrm{mmol})$ in $\mathrm{CH}_{2} \mathrm{Cl}_{2}(30 \mathrm{~mL})$ was successively added DMSO (4.2 mL, $59 \mathrm{mmol}$ ), $i \mathrm{Pr}_{2} \mathrm{NEt}(4.1 \mathrm{~mL}, 24 \mathrm{mmol})$, and $\mathrm{SO}_{3} \cdot \mathrm{Py}(1.96 \mathrm{~g}, 12.3 \mathrm{mmol})$ at $0{ }^{\circ} \mathrm{C}$. After being stirred at room temperature for $30 \mathrm{~min}$, the reaction was quenched by addition of saturated aqueous $\mathrm{NaHCO}_{3}$. The mixture was extracted with $\mathrm{Et}_{2} \mathrm{O}$ (3 times). The combined organic layers were washed with water and brine, dried over $\mathrm{MgSO}_{4}$, filtered, and concentrated in vacuo. The crude product was used in the next reaction without further purification.

To a stirred solution of bromo di-tert-butylmalonate $(2.60 \mathrm{~g}, 8.81 \mathrm{mmol})$ in toluene $(50 \mathrm{~mL})$ was added LHMDS (0.5 M solution in toluene, $16.5 \mathrm{~mL}, 8.3 \mathrm{mmol}$ ) at room temperature. After $30 \mathrm{~min}$, the crude aldehyde $\mathbf{S 1}$ in toluene $(10 \mathrm{~mL})$ was added. The reaction was warmed to $40{ }^{\circ} \mathrm{C}$, stirred for $12 \mathrm{~h}$, and subsequently quenched by addition of saturated aqueous $\mathrm{NH}_{4} \mathrm{Cl}$. The mixture was extracted with EtOAc (3 times). The combined organic layers were washed with water and brine, dried over $\mathrm{MgSO}_{4}$, filtered, and concentrated in vacuo. The crude residue was purified by flash chromatography on silica gel (20:1 hexane:EtOAc) to afford epoxide 12 (3.07 g, $94 \%$ yield in 2 steps, single diastereomer) as a colorless oil. $[\alpha]_{\mathrm{D}}{ }^{20}-13.7^{\circ}\left(c 1.01, \mathrm{CHCl}_{3}\right)$; IR $\left(\mathrm{CHCl}_{3}\right) 2977,1743,1369,1250$, $1114,702 \mathrm{~cm}^{-1}$; ${ }^{1} \mathrm{H}$ NMR $\left(400 \mathrm{MHz}, \mathrm{CDCl}_{3}\right) \delta$ 7.73-7.68 (m, 4H), 7.41-7.31 (m, 6H), $3.61(\mathrm{~d}, J=8.2$ $\mathrm{Hz}, 1 \mathrm{H}), 3.30$ (dd, $J=8.2,2.8 \mathrm{~Hz}, 1 \mathrm{H}), 1.83-1.71(\mathrm{~m}, 1 \mathrm{H}), 1.45$ (s, 9H), 1.30 (s, 9H), 1.09 (s, 9H), $0.95(\mathrm{~d}, J=6.8 \mathrm{~Hz}, 3 \mathrm{H}), 0.78(\mathrm{~d}, J=6.8 \mathrm{~Hz}, 3 \mathrm{H}) ;{ }^{13} \mathrm{C}$ NMR $\left(100 \mathrm{MHz}, \mathrm{CDCl}_{3}\right) \delta 164.9,163.6$, 136.2 (2C), 136.1 (2C), 133.9, 133.3, 130.0, 129.5 (2C), 129.4 (2C), 127.4 (2C), 127.3 (2C), 83.4, 83.0, 75.9, 63.8, 61.5, 31.9, 27.8 (3C), 27.7 (3C), 27.1 (3C), 19.8, 19.1, 16.4; HRMS (FAB+) $\mathrm{m} / z$ calcd for $\mathrm{C}_{32} \mathrm{H}_{46} \mathrm{O}_{6} \mathrm{Si}[\mathrm{M}+\mathrm{H}]^{+}: 554.3142$ found 554.3141 .

$(1 R, 4 R, 5 S)-4-$ Isopropyl- $N$-methoxy- $N$-methyl-2-oxo-3,6-dioxabicyclo[3.1.0]hexane-1-carboxami de (11)

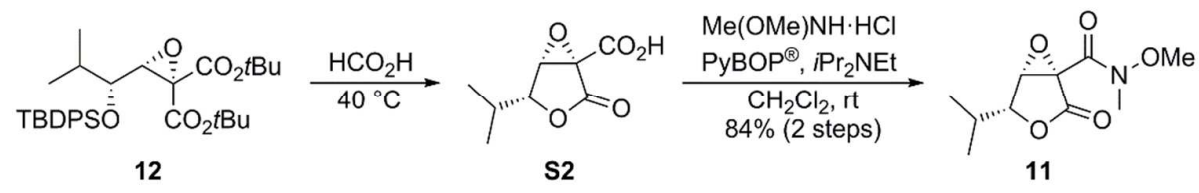


Epoxide 12 (3.07 g, $5.53 \mathrm{mmol})$ was dissolved in formic acid $(30 \mathrm{~mL})$, and the solution was stirred at $40{ }^{\circ} \mathrm{C}$ for $12 \mathrm{~h}$. The reaction mixture was concentrated in vacuo. The crude product was used in the next reaction without further purification.

To a stirred solution of crude carboxylic acid $\mathbf{S 2}$ in $\mathrm{CH}_{2} \mathrm{Cl}_{2}(50 \mathrm{~mL})$ was successively added $N, O$-dimethylhydroxylamine hydrochloride $(1.14 \mathrm{~g}, 11.6 \mathrm{mmol}), i \operatorname{Pr}_{2} \mathrm{NEt}(3.9 \mathrm{~mL}, 22 \mathrm{mmol})$, and $\operatorname{PyBOP}^{\circledR}(5.86 \mathrm{~g}, 11.3 \mathrm{mmol})$ at $0{ }^{\circ} \mathrm{C}$. After stirring at room temperature for $1 \mathrm{~h}$, the reaction was quenched by addition of water. The mixture was extracted with $\mathrm{CH}_{2} \mathrm{Cl}_{2}$ (3 times). The combined organic layers were dried over $\mathrm{MgSO}_{4}$, filtered, and concentrated in vacuo. The crude residue was purified by flash chromatography on silica gel (1:1 hexane:EtOAc). The combined fractions that contained 11 were washed with saturated aqueous $\mathrm{NaHCO}_{3}$, dried over $\mathrm{MgSO}_{4}$, filtered, and concentrated in vacuo to afford weinreb amide 11 (1.06 g, 84\% yield in 2 steps) as a colorless solid. m.p. 65.0-70.3 $\left.{ }^{\circ} \mathrm{C} ;[\alpha]_{\mathrm{D}}{ }^{17}-33.6^{\circ}(c) 1.01, \mathrm{CHCl}_{3}\right)$; IR $\left(\mathrm{CHCl}_{3}\right) 3033,2982,2936,2878,1739,1371$, 1209, 1165, 769, 763, 753, 749, $745 \mathrm{~cm}^{-1}$; ${ }^{1} \mathrm{H}$ NMR (400 MHz, $\left.\mathrm{CDCl}_{3}\right) \delta 4.28(\mathrm{~s}, 1 \mathrm{H}), 4.16(\mathrm{~d}, J=$ $9.0 \mathrm{~Hz}, 1 \mathrm{H}), 3.74(\mathrm{~s}, 3 \mathrm{H}), 3.27(\mathrm{~s}, 3 \mathrm{H}), 2.13-2.03(\mathrm{~m}, 1 \mathrm{H}), 1.10(\mathrm{~d}, J=6.6 \mathrm{~Hz}, 3 \mathrm{H}), 1.08$ (d, $J=6.9$ $\mathrm{Hz}, 3 \mathrm{H}) ;{ }^{13} \mathrm{C} \mathrm{NMR}\left(100 \mathrm{MHz}, \mathrm{CDCl}_{3}\right) \delta 167.1,161.5,83.7,61.2,60.9,58.9,32.2,29.2,19.3,17.8$; HRMS (FAB+) $m / z$ calcd for $\mathrm{C}_{10} \mathrm{H}_{16} \mathrm{NO}[\mathrm{M}+\mathrm{H}]^{+}: 230.1028$ found 230.1033 .

\section{(2S,3S)- $N$-Methoxy- $N$-methyl-3-((R)-2-methyl-1-((triethylsilyl)oxy)propyl)oxirane-2,2-dicarbox amide (17)}

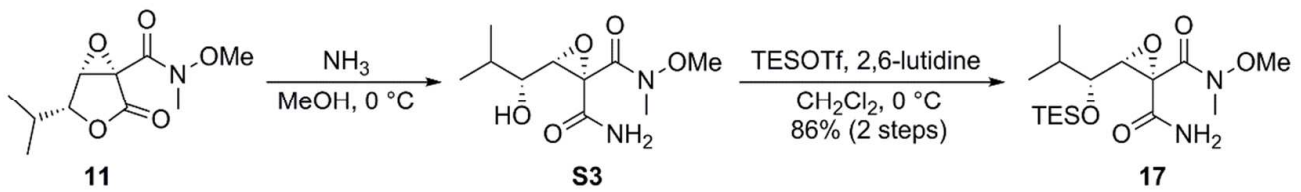

To a stirred solution of weinreb amide $11(2.56 \mathrm{~g}, 11.2 \mathrm{mmol})$ in $\mathrm{MeOH}(30 \mathrm{~mL})$ was added $\mathrm{NH}_{3}$ (7M solution in $\mathrm{MeOH}, 30 \mathrm{~mL}, 210 \mathrm{mmol}$ ) at $0{ }^{\circ} \mathrm{C}$. The solution was stirred at $0{ }^{\circ} \mathrm{C}$ for $1 \mathrm{~h}$ and concentrated in vacuo. The crude product was used in the next reaction without further purification. To a stirred solution of crude alcohol $\mathbf{S 3}$ in $\mathrm{CH}_{2} \mathrm{Cl}_{2}(110 \mathrm{~mL})$ was sequentially added 2,6-lutidine (6.0 $\mathrm{mL}, 56 \mathrm{mmol})$ and TESOTf $(6.0 \mathrm{~mL}, 28 \mathrm{mmol})$ at $0{ }^{\circ} \mathrm{C}$. After being stirred at $0{ }^{\circ} \mathrm{C}$ for $1 \mathrm{~h}$, the reaction mixture was quenched by addition of water. The mixture was extracted with EtOAc (3 times). The combined organic layers were washed successively with $0.5 \mathrm{~N} \mathrm{HCl}$, saturated aqueous $\mathrm{NaHCO}_{3}$, and brine, dried over $\mathrm{MgSO}_{4}$, filtered, and concentrated in vacuo. The crude residue was then dissolved in EtOAc, and silica gel was added. After stirring at $40{ }^{\circ} \mathrm{C}$ for $36 \mathrm{~h}$, the mixture was filtered through celite and concentrated in vacuo. The crude residue was purified by flash chromatography on silica gel (1:1 hexane:EtOAc) to afford amide 17 (3.46 g, 86\% yield in 2 steps) as a colorless solid. m.p. $\left.121.2-128.1^{\circ} \mathrm{C} ;[\alpha]_{\mathrm{D}}{ }^{22}-32.0^{\circ}(c) 1.15, \mathrm{CHCl}_{3}\right)$; IR $\left(\mathrm{CHCl}_{3}\right) 3519,3402$, 2961, 2878, 1702, 1674, 1571, 1462, 1390, $1062 \mathrm{~cm}^{-1} ;{ }^{1} \mathrm{H}$ NMR (400 MHz, $\left.\mathrm{CDCl}_{3}\right) \delta 6.43$ (br s, 1H), 
6.15 (br s, 1H), 3.74 (s, 3H), 3.54 (d, $J=8.6 \mathrm{~Hz}, 1 \mathrm{H}), 3.35$ (dd, $J=8.6,4.8 \mathrm{~Hz}, 1 \mathrm{H}), 3.19$ (s, 3H), $1.83-1.66(\mathrm{~m}, 1 \mathrm{H}), 0.94(\mathrm{t}, J=9.0 \mathrm{~Hz}, 9 \mathrm{H}), 0.94(\mathrm{~d}, J=6.4 \mathrm{~Hz}, 3 \mathrm{H}), 0.90(\mathrm{~d}, J=6.8 \mathrm{~Hz}, 3 \mathrm{H})$, 0.68-0.52 (m, 6H); ${ }^{13} \mathrm{C}$ NMR (100 MHz, $\left.\mathrm{CDCl}_{3}\right) \delta 168.3,165.5,74.8,65.6,63.0,61.5,32.6,32.3$, 18.7, 16.8, 6.8 (3C), $5.1(3 \mathrm{C})$; HRMS (FAB+) $\mathrm{m} / z$ calcd for $\mathrm{C}_{16} \mathrm{H}_{32} \mathrm{~N}_{2} \mathrm{O}_{5} \mathrm{Si}[\mathrm{M}+\mathrm{H}]^{+}: 360.2159$ found 361.2160 .

\section{Dimethyl}

(2-((2R,3S)-2-carbamoyl-3-((R)-2-methyl-1-((triethylsilyl)oxy)propyl)oxiran-2-yl)-2-oxoethyl)p hosphonate (9)

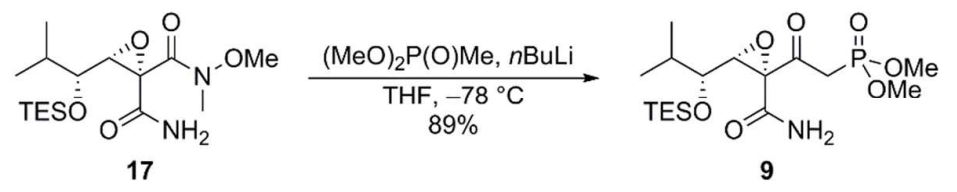

To a stirred solution of dimethyl methylphosphonate $(3.57 \mathrm{~g}, 28.8 \mathrm{mmol})$ in THF $(150 \mathrm{~mL})$ was added $n \mathrm{BuLi}(2.65 \mathrm{M}$ solution in hexane, $9.0 \mathrm{~mL}, 24 \mathrm{mmol})$ dropwise via syringe at $-78{ }^{\circ} \mathrm{C}$. After stirring at $-78{ }^{\circ} \mathrm{C}$ for $30 \mathrm{~min}$, amide $17(3.46 \mathrm{~g}, 9.60 \mathrm{mmol})$ in $\mathrm{THF}(70 \mathrm{~mL})$ was added. After $30 \mathrm{~min}$, the reaction was quenched by addition of saturated aqueous $\mathrm{NH}_{4} \mathrm{Cl}$. The mixture was extracted with EtOAc (3 times). The combined organic layers were dried over $\mathrm{MgSO}_{4}$ and concentrated in vacuo. The crude residue was purified by flash chromatography on silica gel (EtOAc) to afford phosphonate 9 (3.61 g, 89\% yield) as a colorless oil. $[\alpha]_{\mathrm{D}}{ }^{20}-20.3^{\circ}\left(c\right.$ 1.02, $\left.\mathrm{CHCl}_{3}\right)$; IR (neat) 3495, 3401, 2960, 2878, 1714, 1210, 1059, $1038 \mathrm{~cm}^{-1}$; ${ }^{1} \mathrm{H}$ NMR (400 MHz, $\left.\mathrm{CDCl}_{3}\right) \delta 6.88$ (br s, 1H), 5.88 (br s, $1 \mathrm{H}$ ), $3.78(\mathrm{~d}, J=11.4 \mathrm{~Hz}, 3 \mathrm{H}), 3.77(\mathrm{~d}, J=11.4 \mathrm{~Hz}, 3 \mathrm{H}), 3.42-3.27(\mathrm{~m}, 2 \mathrm{H}), 3.40(\mathrm{dd}, J=4.4,7.8 \mathrm{~Hz}$, $1 \mathrm{H}), 3.32(\mathrm{~d}, J=7.8 \mathrm{~Hz}, 1 \mathrm{H}), 1.77-1.67(\mathrm{~m}, 1 \mathrm{H}), 0.95(\mathrm{t}, J=8.0 \mathrm{~Hz}, 9 \mathrm{H}), 0.89(\mathrm{~d}, J=6.8 \mathrm{~Hz}, 3 \mathrm{H})$, $0.87(\mathrm{~d}, J=6.8 \mathrm{~Hz}, 3 \mathrm{H}), 0.68-0.52(\mathrm{~m}, 6 \mathrm{H}) ;{ }^{13} \mathrm{C} \mathrm{NMR}\left(100 \mathrm{MHz}, \mathrm{CDCl}_{3}\right) \delta 196.7^{*}, 165.0,74.3,65.2$, $53.3^{*}, 53.2^{*}, 36.8,35.5,32.2,18.9,16.7,6.8(3 \mathrm{C}), 5.0(3 \mathrm{C})$ ( $^{*}$ The signals are split into a doublet by phosphorus atom.); HRMS (FAB+) $m / z$ calcd for $\mathrm{C}_{17} \mathrm{H}_{35} \mathrm{NO}_{7} \mathrm{PSi}[\mathrm{M}+\mathrm{H}]^{+}: 424.1920$ found 424.1911 .

\section{Methyl $(4 R, 6 R, E)-2$-bromo-4,6-dimethyloct-2-enoate $((R, R)-19)$}

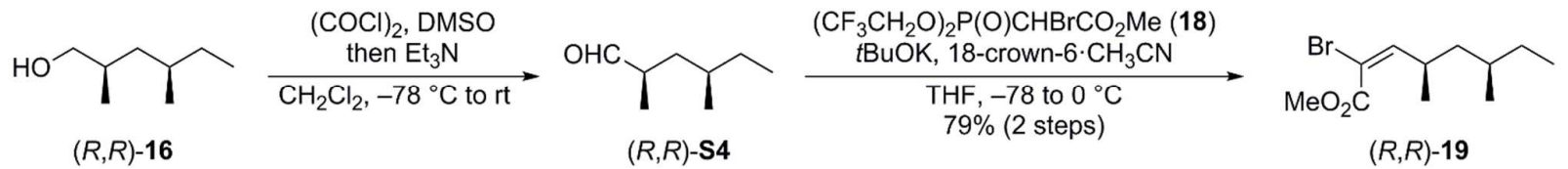

To a stirred solution of $(\mathrm{COCl})_{2}(0.52 \mathrm{~mL}, 6.1 \mathrm{mmol})$ in $\mathrm{CH}_{2} \mathrm{Cl}_{2}(10 \mathrm{~mL})$ was slowly added DMSO $(0.64 \mathrm{~mL}, 9.01 \mathrm{mmol})$ in $\mathrm{CH}_{2} \mathrm{Cl}_{2}(10 \mathrm{~mL})$ via syringe at $-78^{\circ} \mathrm{C}$. The resulting mixture was stirred for $30 \mathrm{~min}$, and alcohol $(R, R)-\mathbf{1 6}(391 \mathrm{mg}, 3.00 \mathrm{mmol})$ in $\mathrm{CH}_{2} \mathrm{Cl}_{2}(10 \mathrm{~mL})$ was carefully added dropwise. 
After stirring for $30 \mathrm{~min}, \mathrm{Et}_{3} \mathrm{~N}(2.1 \mathrm{~mL}, 15 \mathrm{mmol})$ was added in a single portion. The cooling bath was removed, and the reaction was allowed to warm to room temperature. After $30 \mathrm{~min}$, the reaction mixture was diluted with pentane and brine, and extracted with pentane ( 3 times). The combined organic layers were washed with brine, dried over $\mathrm{Na}_{2} \mathrm{SO}_{4}$, filtered, and concentrated in vacuo. The crude product was used in the next reaction without further purification.

A solution of 18-crown-6. $\mathrm{CH}_{3} \mathrm{CN}(1.20 \mathrm{~g}, 3.93 \mathrm{mmol})$ in THF (40 mL) was added to methyl 2-(bis(2,2,2-trifluoroethoxy)phosphoryl)-2-bromoacetate $(\mathbf{1 8}, 1.55 \mathrm{~g}, 3.90 \mathrm{mmol})$ in THF (10 mL) at room temperature. The mixture was cooled to $-78{ }^{\circ} \mathrm{C}$, and $t \mathrm{BuOK}(1.0 \mathrm{M}$ solution in $\mathrm{THF}, 3.9 \mathrm{ml}$, $3.9 \mathrm{mmol})$ was added. After stirring for $30 \mathrm{~min}$, the crude aldehyde $(R, R)-\mathbf{S} 4$ in THF $(10 \mathrm{~mL})$ was added dropwise. After complete addition, the reaction mixture was allowed to warm to $0{ }^{\circ} \mathrm{C}$ and stirred for $1 \mathrm{~h}$. The reaction was quenched by addition of saturated aqueous $\mathrm{NH}_{4} \mathrm{Cl}$, and the mixture was extracted with EtOAc (3 times). The combined organic layers were washed with water and brine, dried over $\mathrm{MgSO}_{4}$, filtered, and concentrated in vacuo. The crude residue was purified by flash chromatography on silica gel (50:1 hexane:EtOAc) to afford $(E)$ - $\alpha$-bromoacrylate $(R, R)-19(624 \mathrm{mg}$, $79 \%$ yield in 2 steps $)$ as a colorless oil. $[\alpha]_{\mathrm{D}}{ }^{23}-18.8^{\circ}\left(c 1.15, \mathrm{CHCl}_{3}\right)$; IR $\left(\mathrm{CHCl}_{3}\right) 3025,2963,2928$, $2875,1720,1236,1224,1222,1216,778,775,740 \mathrm{~cm}^{-1} ;{ }^{1} \mathrm{H}$ NMR $\left(300 \mathrm{MHz}, \mathrm{CDCl}_{3}\right) \delta 6.39(\mathrm{~d}, J=$ $10.2 \mathrm{~Hz}, 1 \mathrm{H}), 3.82(\mathrm{~s}, 3 \mathrm{H}), 3.34-3.24(\mathrm{~m}, 1 \mathrm{H}), 1.37-1.22(\mathrm{~m}, 3 \mathrm{H}), 1.18-1.06(\mathrm{~m}, 2 \mathrm{H}), 1.02(\mathrm{~d}, J=6.6$ $\mathrm{Hz}, 3 \mathrm{H}), 0.86(\mathrm{t}, J=6.9 \mathrm{~Hz}, 3 \mathrm{H}), 0.82(\mathrm{~d}, J=6.3 \mathrm{~Hz}, 3 \mathrm{H}) ;{ }^{13} \mathrm{C} \mathrm{NMR}\left(100 \mathrm{MHz}, \mathrm{CDCl}_{3}\right) \delta 163.5$, $154.5,109.2,52.8,44.2,33.7,32.4,29.9,20.5,18.9,11.2$; HRMS (EI+) $m / z$ calcd for $\mathrm{C}_{11} \mathrm{H}_{19} \mathrm{BrO}_{2}$ $[\mathrm{M}]^{+}: 262.0568$ found 262.0565 .

\section{Methyl (4S,6S,E)-2-bromo-4,6-dimethyloct-2-enoate $((S, S)-19)$}

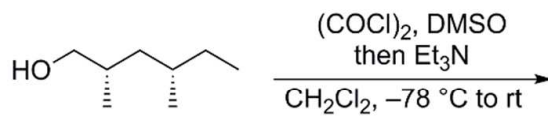

$(S, S)-16$

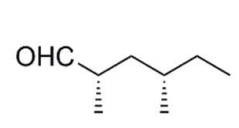

$(S, S)-S 4$

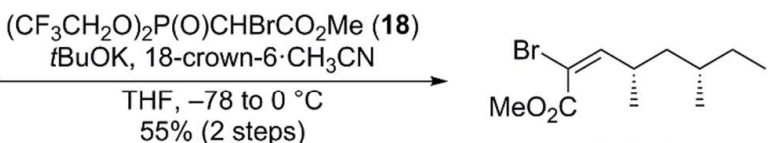

$(S, S)-19$

(E)- $\alpha$-bromoacrylate $(S, S)-23$ (436 mg, 55\% yield in 2 steps, colorless oil) was obtained from alcohol $(S, S)$-16 $(391 \mathrm{mg}, 3.00 \mathrm{mmol})$ by using a procedure similar to that for the $(R, R)$-isomer. The spectroscopic data was identical to that of $(R, R)-\mathbf{2 3}$ except for optical rotation $\left([\alpha]_{\mathrm{D}}^{23}+17.9^{\circ}(c 0.75\right.$, $\mathrm{CHCl}_{3}$ )).

$(4 R, 6 R, E)-2-B r o m o-4,6-d i m e t h y l o c t-2-e n-1-o l ~((R, R)-20)$

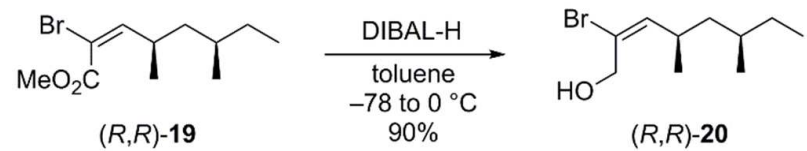


To a stirred solution of $(E)$ - $\alpha$-bromoacrylate $(R, R)-19(1.07 \mathrm{~g}, 4.07 \mathrm{mmol})$ in toluene $(40 \mathrm{~mL})$ was added DIBAL-H $(1.02 \mathrm{M}$ solution in hexane $10 \mathrm{~mL}, 10 \mathrm{mmol})$ at $-78{ }^{\circ} \mathrm{C}$, and the reaction mixture was allowed to warm to $0{ }^{\circ} \mathrm{C}$. After being stirred for $1 \mathrm{~h}$, the reaction was quenched by addition of $\mathrm{MeOH}$ and saturated aqueous Rochelle salt, and vigorously stirred for $1 \mathrm{~h}$. The mixture was extracted with EtOAc (3 times). The combined organic layers were washed with water and brine, dried over $\mathrm{Na}_{2} \mathrm{SO}_{4}$, filtered, and concentrated in vacuo. The crude residue was purified by flash chromatography on silica gel (20:1 hexane:EtOAc) to afford alcohol $(R, R)-\mathbf{2 0}$ (865 $\mathrm{mg}, 90 \%$ yield) as a colorless oil. $[\alpha]_{\mathrm{D}}{ }^{23}-28.9^{\circ}\left(c 1.15, \mathrm{CHCl}_{3}\right)$; IR $\left(\mathrm{CHCl}_{3}\right) 3592,2963,2928,2875,1462,1454$, 1380, 1039, 988, 751, 739, $671 \mathrm{~cm}^{-1} ;{ }^{1} \mathrm{H}$ NMR (300 MHz, $\left.\mathrm{CDCl}_{3}\right) \delta 5.76(\mathrm{~d}, J=10.5 \mathrm{~Hz}, 1 \mathrm{H}), 4.31$ $(\mathrm{d}, J=6.6 \mathrm{~Hz}, 2 \mathrm{H}), 2.62-2.53(\mathrm{~m}, 1 \mathrm{H}), 1.83(\mathrm{t}, J=6.6 \mathrm{~Hz}, 1 \mathrm{H}), 1.33-1.22(\mathrm{~m}, 3 \mathrm{H}), 1.19-1.04(\mathrm{~m}$, 2H), $1.00(\mathrm{~d}, J=6.6 \mathrm{~Hz}, 3 \mathrm{H}), 0.86(\mathrm{t}, J=7.2 \mathrm{~Hz}, 3 \mathrm{H}), 0.83(\mathrm{~d}, J=6.3 \mathrm{~Hz}, 3 \mathrm{H}) ;{ }^{13} \mathrm{C} \mathrm{NMR}(100 \mathrm{MHz}$, $\left.\mathrm{CDCl}_{3}\right) \delta 141.3,123.2,62.9,44.2,32.6,32.0,29.9,21.6,18.8,11.2$; HRMS (EI+) $\mathrm{m} / z$ calcd for $\mathrm{C}_{10} \mathrm{H}_{19} \mathrm{BrO}[\mathrm{M}]^{+}: 234.0619$ found 234.0615.

\section{$(4 S, 6 S, E)-2-B r o m o-4,6-d i m e t h y l o c t-2-e n-1-o l ~((S, S)-20)$}

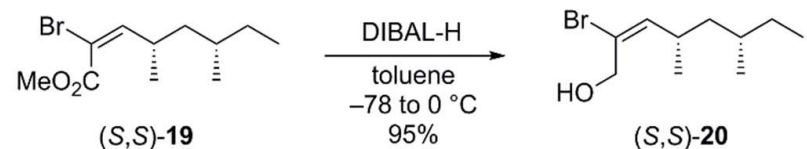

Alcohol $(S, S)$-20 (1.39 g, 95\% yield, colorless oil) was obtained from $(S, S)-19$ (1.64 g, $6.24 \mathrm{mmol})$ by using a procedure similar to that for the $(R, R)$-isomer. The spectroscopic data was identical to that of $(R, R)-\mathbf{2 0}$ except for optical rotation $\left([\alpha]_{\mathrm{D}}^{23}+29.3^{\circ}\left(c 0.38, \mathrm{CHCl}_{3}\right)\right)$.

\section{$(4 R, 6 R, E)-2-B r o m o-4,6-$ dimethyloct-2-ene $((R, R)-15)$}

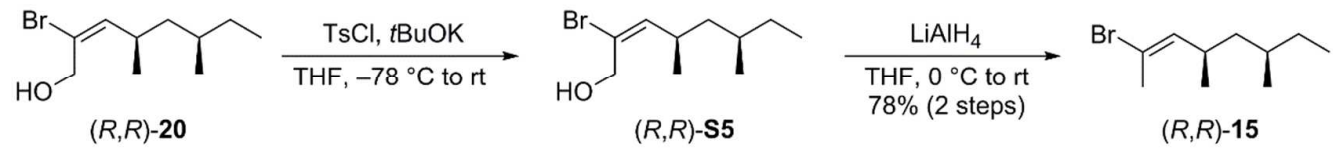

To a stirred solution of alcohol $(R, R)-\mathbf{2 0}(2.61 \mathrm{~g}, 11.1 \mathrm{mmol})$ in THF $(110 \mathrm{~mL})$ was added $t \mathrm{BuOK}$ $(1.0 \mathrm{M}$ solution in THF, $13.3 \mathrm{~mL}, 13.3 \mathrm{mmol})$ dropwise via syringe at $-78{ }^{\circ} \mathrm{C}$. The solution was stirred at $-78{ }^{\circ} \mathrm{C}$ for $30 \mathrm{~min}$, and $\mathrm{TsCl}(2.54 \mathrm{~g}, 13.3 \mathrm{mmol})$ was then added in small portions. After 10 min, cooling bath was removed, and the reaction was allowed to warm to room temperature. The reaction was quenched by addition of water and stirred for $5 \mathrm{~min}$, and the mixture was extracted with $\mathrm{Et}_{2} \mathrm{O}$ (3 times). The combined organic layers were washed successively with $0.5 \mathrm{~N} \mathrm{HCl}$, saturated aqueous $\mathrm{NaHCO}_{3}$, and brine, dried over $\mathrm{Na}_{2} \mathrm{SO}_{4}$, filtered, and concentrated in vacuo. The crude product was used in the next reaction without further purification. 
To a stirred suspension of $\mathrm{LiAlH}_{4}(687 \mathrm{mg}, 16.7 \mathrm{mmol})$ in THF $(100 \mathrm{~mL})$ was added the crude tosylate $(R, R)-\mathbf{S 5}$ in THF $(10 \mathrm{~mL})$ at $0{ }^{\circ} \mathrm{C}$. The mixture was allowed to warm to room temperature and stirred for $30 \mathrm{~min}$. The reaction was quenched by addition of cold saturated aqueous $\mathrm{Na}_{2} \mathrm{SO}_{4}$ $(100 \mathrm{~mL})$. After stirring for $1 \mathrm{~h}$, the mixture was extracted with pentane (3 times). The combined organic layers were dried over $\mathrm{MgSO}_{4}$, filtered, and concentrated in vacuo. The crude residue was purified by flash chromatography on silica gel (pentane) to afford vinyl bromide $(R, R)-15(1.90 \mathrm{~g}$, $78 \%$ yield in 2 steps $)$ as a colorless oil. $[\alpha]_{\mathrm{D}}{ }^{23}-31.7^{\circ}\left(c 1.32, \mathrm{CHCl}_{3}\right)$; IR $\left(\mathrm{CHCl}_{3}\right) 3676,3023,3013$, 2963, 2927, 2874, 2360, 1740, 1652, 1458, 1381, 1223, 1218, 1214, 1209, 789, 783, 773, 748, 736, $668 \mathrm{~cm}^{-1} ;{ }^{1} \mathrm{H}$ NMR $\left(300 \mathrm{MHz}, \mathrm{CDCl}_{3}\right) \delta 5.58(\mathrm{dq}, J=10.2,1.2 \mathrm{~Hz}, 1 \mathrm{H}), 2.48-2.38(\mathrm{~m}, 1 \mathrm{H}), 2.23(\mathrm{~d}$, $J=1.2 \mathrm{~Hz}, 3 \mathrm{H}), 1.35-1.21(\mathrm{~m}, 3 \mathrm{H}), 1.17-0.99(\mathrm{~m}, 2 \mathrm{H}), 0.95(\mathrm{~d}, J=6.6 \mathrm{~Hz}, 3 \mathrm{H}), 0.86(\mathrm{t}, J=7.5 \mathrm{~Hz}$, $3 \mathrm{H}), 0.83(\mathrm{~d}, J=6.3 \mathrm{~Hz}, 3 \mathrm{H}) ;{ }^{13} \mathrm{C} \mathrm{NMR}\left(100 \mathrm{MHz}, \mathrm{CDCl}_{3}\right) \delta 138.6,117.9,44.3,32.5,32.1,30.0$, 23.4, 21.2, 19.0, 11.2; HRMS (EI+) $m / z$ calcd for $\mathrm{C}_{10} \mathrm{H}_{19} \mathrm{Br}[\mathrm{M}]^{+}: 218.0670$ found 218.0669 .

\section{$(4 S, 6 S, E)-2-B r o m o-4,6-d i m e t h y l o c t-2-e n e ~((S, S)-15)$}

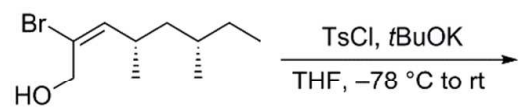

$(S, S)-20$

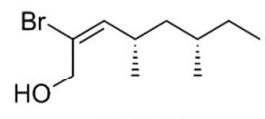

$(S, S)-S 5$

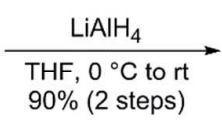

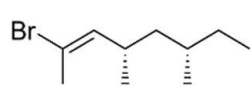

$(S, S)-15$

Vinyl bromide $(S, S)$-15 (1.13 g, 90\% yield in 2 steps, colorless oil) was obtained from $(S, S)$-20 (1.39 $\mathrm{g}, 5.91 \mathrm{mmol})$ by using a procedure similar to that for the $(R, R)$-isomer. The spectroscopic data was identical to that of $(R, R)-\mathbf{1 5}$ except for optical rotation $\left([\alpha]_{\mathrm{D}}{ }^{19}+54.3^{\circ}\left(c 1.25, \mathrm{CHCl}_{3}\right)\right)$.

\section{$(2 E, 4 E, 6 E, 8 R, 10 R)-6,8,10-T r i m e t h y l d o d e c a-2,4,6-t r i e n-1-o l ~((R, R)-21)$}

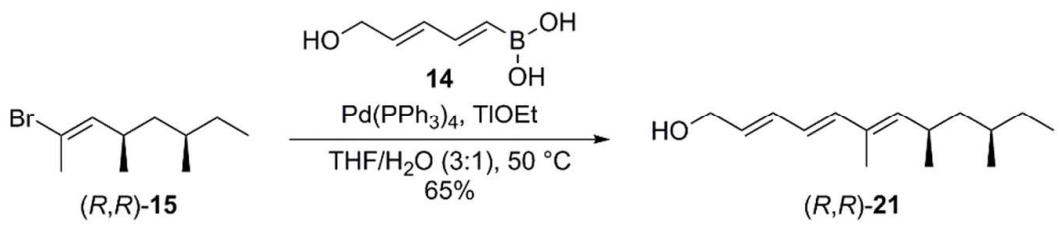

(All operations were performed in the dark.) A solution of vinyl bromide $(R, R)-15(1.04 \mathrm{~g}, 4.75$ $\mathrm{mmol})$ and bronic acid $14(3.04 \mathrm{~g}, 23.8 \mathrm{mmol})$ in THF $(36 \mathrm{~mL})$ and water $(12 \mathrm{~mL})$ was thoroughly degassed by the freeze-pump-thaw procedure. $\mathrm{Pd}\left(\mathrm{PPh}_{3}\right)_{4}(549 \mathrm{mg}, 0.475 \mathrm{mmol})$ was added, and the reaction mixture was stirred at $50{ }^{\circ} \mathrm{C}$ for $5 \mathrm{~min}$. TlOEt $(0.6 \mathrm{~mL}, 8.5 \mathrm{mmol})$ was added, and the stirring was continued for $30 \mathrm{~min}$. The reaction mixture was diluted with $\mathrm{Et}_{2} \mathrm{O}$ and $1 \mathrm{~N}$ aqueous $\mathrm{NaHSO}_{4}(20 \mathrm{~mL})$, and filtered through celite. The organic layer was separated, washed with water and brine, dried over $\mathrm{Na}_{2} \mathrm{SO}_{4}$, filtered, and concentrated in vacuo. The crude residue was purified by flash chromatography on silica gel (5:1 hexane:EtOAc) to afford triene alcohol $(R, R)-21$ (691 mg, 
$65 \%$ yield $)$ as a colorless oil. $[\alpha]_{\mathrm{D}}^{22}-21.2^{\circ}\left(c 1.03, \mathrm{CHCl}_{3}\right)$; IR $\left(\mathrm{CHCl}_{3}\right) 3613,3013,2963,2925$, 2874, 1457, 1379, 1213, 987, 888, 739, 734, $730 \mathrm{~cm}^{-1} ;{ }^{1} \mathrm{H}$ NMR $\left(300 \mathrm{MHz}, \mathrm{CDCl}_{3}\right) \delta$ 6.34-6.09 (m, $3 \mathrm{H}), 5.84(\mathrm{dt}, J=5.9,7.8 \mathrm{~Hz}, 1 \mathrm{H}), 5.26(\mathrm{~d}, J=9.6 \mathrm{~Hz}, 1 \mathrm{H}), 4.20(\mathrm{t}, J=5.9 \mathrm{~Hz}, 2 \mathrm{H}), 2.65-2.55(\mathrm{~m}$, $1 \mathrm{H}), 1.78(\mathrm{~s}, 3 \mathrm{H}), 1.31-1.24(\mathrm{~m}, 3 \mathrm{H}), 1.18-1.03(\mathrm{~m}, 2 \mathrm{H}), 0.95(\mathrm{~d}, J=6.3 \mathrm{~Hz}, 3 \mathrm{H}), 0.84(\mathrm{t}, J=7.2 \mathrm{~Hz}$, $3 \mathrm{H}), 0.81(\mathrm{~d}, J=6.3 \mathrm{~Hz}, 3 \mathrm{H})$. A signal due to one proton $(\mathrm{OH})$ was not observed; ${ }^{13} \mathrm{C}$ NMR $(100$ $\left.\mathrm{MHz}, \mathrm{CDCl}_{3}\right) \delta 141.1,138.7,132.5,132.0,130.4,125.2,63.6,44.9,32.2,30.4,30.1,21.5,19.1,12.5$, 11.3; HRMS (EI+) $m / z$ calcd for $\mathrm{C}_{15} \mathrm{H}_{26} \mathrm{O}[\mathrm{M}]^{+}: 222.1984$ found 222.1978.

$(2 E, 4 E, 6 E, 8 S, 10 S)-6,8,10-T r i m e t h y l d o d e c a-2,4,6-t r i e n-1-o l ~((S, S)-21)$

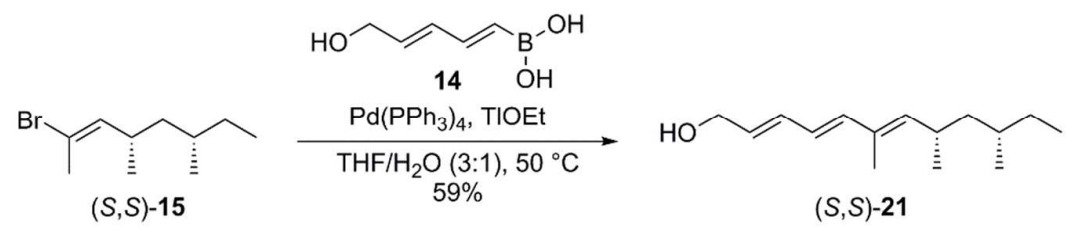

Alcohol $(S, S)$-21 (136 mg, 59\% yield, colorless oil) was obtained from $(S, S)$-15 (229 mg, $1.04 \mathrm{mmol})$ by using a procedure similar to that for the $(R, R)$-isomer. The spectroscopic data was identical to that of $(R, R)-21$ except for optical rotation $\left([\alpha]_{\mathrm{D}}{ }^{18}+36.1^{\circ}\left(c 1.00, \mathrm{CHCl}_{3}\right)\right)$.

\section{$(2 E, 4 E, 6 E, 8 R, 10 R)-6,8,10-T r i m e t h y l d o d e c a-2,4,6$-trienal $((R, R)-10)$}

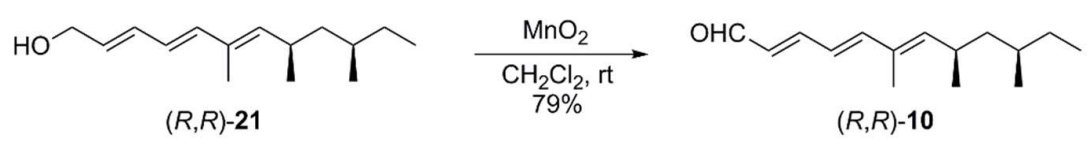

(All operations were performed in the dark.) To a stirred solution of triene alcohol $(R, R)-\mathbf{2 1}(660 \mathrm{mg}$, $2.99 \mathrm{mmol})$ in $\mathrm{CH}_{2} \mathrm{Cl}_{2}(30 \mathrm{~mL})$ was added $\mathrm{MnO}_{2}(7.86 \mathrm{~g}, 89.8 \mathrm{mmol})$ at $0{ }^{\circ} \mathrm{C}$. After $1 \mathrm{~h}$ at room temperature, the reaction mixture was filtered through celite and concentrated in vacuo. The crude residue was purified by flash chromatography on silica gel (20:1 hexane:EtOAc) to afford triene aldehyde $(R, R)-\mathbf{1 0}\left(521 \mathrm{mg}, 79 \%\right.$ yield) as a yellow oil. $[\alpha]_{\mathrm{D}}{ }^{21}-37.8^{\circ}\left(\mathrm{c} 1.13, \mathrm{CHCl}_{3}\right)$; IR $\left(\mathrm{CHCl}_{3}\right)$ 3027, 3014, 2964, 2926, 2874, 2741, 1673, 1603, 1218, 1160, 1132, 985, 776, 741, 671, $480 \mathrm{~cm}^{-1} ;{ }^{1} \mathrm{H}$ NMR $\left(300 \mathrm{MHz}, \mathrm{CDCl}_{3}\right) \delta 9.55(\mathrm{~d}, J=8.0 \mathrm{~Hz}, 1 \mathrm{H}), 7.15(\mathrm{dd}, J=15.3,11.0 \mathrm{~Hz}, 1 \mathrm{H}), 6.68(\mathrm{~d}, J=$ $15.3 \mathrm{~Hz}, 1 \mathrm{H}), 6.36(\mathrm{dd}, J=15.3,11.0 \mathrm{~Hz}, 1 \mathrm{H}), 6.16(\mathrm{dd}, J=15.3,8.0 \mathrm{~Hz}, 1 \mathrm{H}), 5.55(\mathrm{~d}, J=10.2 \mathrm{~Hz}$, $1 \mathrm{H}), 2.72-2.59(\mathrm{~m}, 1 \mathrm{H}), 1.83(\mathrm{~d}, J=0.9 \mathrm{~Hz}, 3 \mathrm{H}), 1.37-1.20(\mathrm{~m}, 3 \mathrm{H}), 1.17-1.10(\mathrm{~m}, 2 \mathrm{H}), 0.98(\mathrm{~d}, J=$ $6.6 \mathrm{~Hz}, 3 \mathrm{H}), 0.85(\mathrm{t}, J=8.4 \mathrm{~Hz}, 3 \mathrm{H}), 0.83(\mathrm{~d}, J=6.0 \mathrm{~Hz}, 3 \mathrm{H}) ;{ }^{13} \mathrm{C} \mathrm{NMR}\left(100 \mathrm{MHz}, \mathrm{CDCl}_{3}\right) \delta 193.7$, $153.3,148.4,147.4,132.3,130.4,123.8,44.6,32.4,30.9,30.1,21.7,19.1,12.4$, 11.3; HRMS (FAB+) $m / z$ calcd for $\mathrm{C}_{15} \mathrm{H}_{24} \mathrm{O}[\mathrm{M}+\mathrm{H}]^{+}: 221.1827$ found 221.1902. 
$(2 E, 4 E, 6 E, 8 S, 10 S)-6,8,10-T r i m e t h y l d o d e c a-2,4,6-t r i e n a l ~((S, S)-10)$

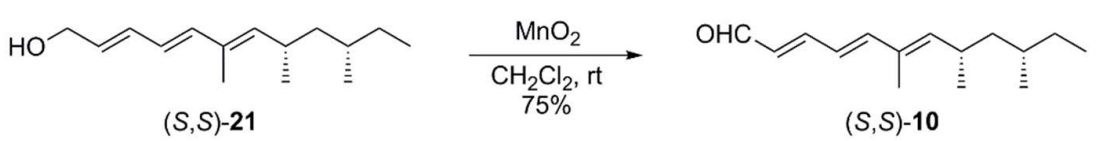

Aldehyde $(S, S)$-10 (100 mg, 75\% yield, yellow oil) was obtained from $(S, S)$-21 (136 mg, 0.454 $\mathrm{mmol})$ by using a procedure similar to that for the $(R, R)$-isomer. The spectroscopic data was identical to that of $(R, R)-10$ except for optical rotation $\left([\alpha]_{\mathrm{D}}{ }^{16}+56.0^{\circ}\left(c 0.97, \mathrm{CHCl}_{3}\right)\right)$.

$(2 R, 3 R)-3-((R)-2-M e t h y l-1-((t r i e t h y l s i l y l) o x y) p r o p y l)-2-((2 E, 4 E, 6 E, 8 E, 10 R, 12 R)-8,10,12-t r i m e t$ hyltetradeca-2,4,6,8-tetraenoyl)oxirane-2-carboxamide $((R, R)-S 6)$

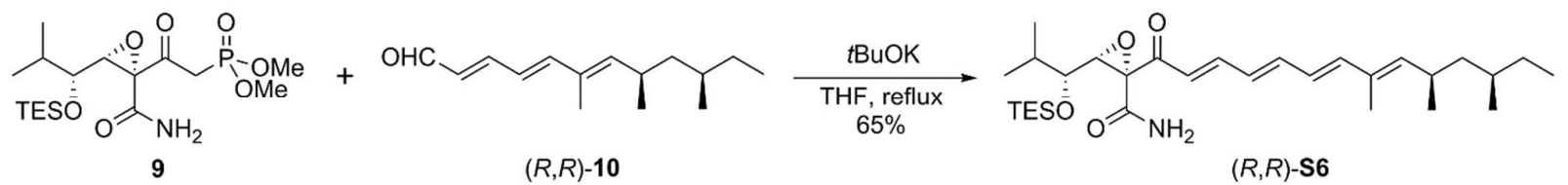

(All operations were performed in the dark.) To a stirred solution of phosphonate 9 (597 $\mathrm{mg}, 1.41$ mmol) in THF $(1.0 \mathrm{~mL})$ was added $t$ BuOK $(1.0 \mathrm{M}$ solution in THF, $1.2 \mathrm{~mL}, 1.2 \mathrm{mmol})$ at room temperature. After stirring for $30 \mathrm{~min}$, aldehyde $(R, R)-\mathbf{1 0}(222 \mathrm{mg}, 1.00 \mathrm{mmol})$ in THF $(1.0 \mathrm{~mL})$ was added dropwise. After complete addition, the reaction mixture was heated to reflux for $10 \mathrm{~h}$. The reaction was quenched by addition of saturated aqueous $\mathrm{NH}_{4} \mathrm{Cl}$, and the mixture was extracted with EtOAc (3 times). The combined organic layers were washed with water and brine, dried over $\mathrm{MgSO}_{4}$, filtered, and concentrated in vacuo. The crude residue was purified by flash chromatography on silica gel (5:1 hexane:EtOAc) to afford tetraene amide $(R, R)-\mathbf{S 6}(339 \mathrm{mg}, 65 \%$ yield) as a yellow amorphous. $[\alpha]_{\mathrm{D}}{ }^{20}-8.5^{\circ}\left(c 0.57, \mathrm{CHCl}_{3}\right)$; IR (neat) $3417,3327,2960,2876,1703,1552,1462,1351$ $\mathrm{cm}^{-1}$; ${ }^{1} \mathrm{H}$ NMR (400 MHz, $\left.\mathrm{CDCl}_{3}\right) \delta 7.53$ (br d, $\left.J=3.0 \mathrm{~Hz}, 1 \mathrm{H}\right), 7.51$ (dd, $\left.J=15.0,11.7 \mathrm{~Hz}, 1 \mathrm{H}\right)$, $6.77(\mathrm{dd}, J=11.1,15.0 \mathrm{~Hz}, 1 \mathrm{H}), 6.56(\mathrm{~d}, J=15.0 \mathrm{~Hz}, 1 \mathrm{H}), 6.50(\mathrm{~d}, J=15.2 \mathrm{~Hz}, 1 \mathrm{H}), 6.38(\mathrm{dd}, J=$ 15.0, 11.7 Hz, 1H), 6.26 (dd, $J=15.2,11.1 \mathrm{~Hz}, 1 \mathrm{H}), 5.69$ (br d, $J=3.0 \mathrm{~Hz}, 1 \mathrm{H}), 5.44$ (d, $J=9.6 \mathrm{~Hz}$, $1 \mathrm{H}), 3.51(\mathrm{dd}, J=8.1,5.0 \mathrm{~Hz}, 1 \mathrm{H}), 3.17(\mathrm{~d}, J=8.1 \mathrm{~Hz}, 1 \mathrm{H}), 2.69-2.57(\mathrm{~m}, 1 \mathrm{H}), 1.81(\mathrm{~s}, 3 \mathrm{H})$, $1.81-1.71(\mathrm{~m}, 2 \mathrm{H}), 1.34-1.20(\mathrm{~m}, 3 \mathrm{H}), 1.16-1.06(\mathrm{~m}, 1 \mathrm{H}), 1.00(\mathrm{t}, J=7.6 \mathrm{~Hz}, 9 \mathrm{H}), 0.96(\mathrm{~d}, J=6.8 \mathrm{~Hz}$, $3 \mathrm{H}), 0.92(\mathrm{~d}, J=6.8 \mathrm{~Hz}, 3 \mathrm{H}), 0.89$ (d, $J=6.8 \mathrm{~Hz}, 3 \mathrm{H}), 0.84(\mathrm{t}, J=7.6 \mathrm{~Hz}, 3 \mathrm{H}), 0.82$ (d, $J=5.6 \mathrm{~Hz}$, $3 \mathrm{H}), 0.77-0.63(\mathrm{~m}, 6 \mathrm{H}) ;{ }^{13} \mathrm{C} \mathrm{NMR}\left(100 \mathrm{MHz}, \mathrm{CDCl}_{3}\right) \delta 193.7,165.8,147.6,145.9,145.3,144.7$, $132.5,129.0,125.5,120.5,74.2,65.5,64.5,44.7,32.4,32.3,30.8,30.1,21.3,19.1,18.8,17.1,12.4$, 11.3, 6.8 (3C), $5.0(3 \mathrm{C})$; HRMS (EI+) $m / z$ calcd for $\mathrm{C}_{30} \mathrm{H}_{51} \mathrm{NO}_{4} \mathrm{Si}[\mathrm{M}]^{+}: 517.3587$ found 517.3582.

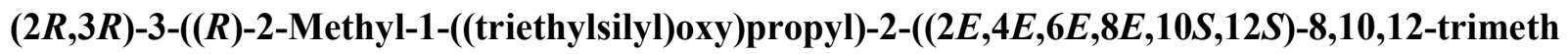


yltetradeca-2,4,6,8-tetraenoyl)oxirane-2-carboxamide $((S, S)-S 6)$

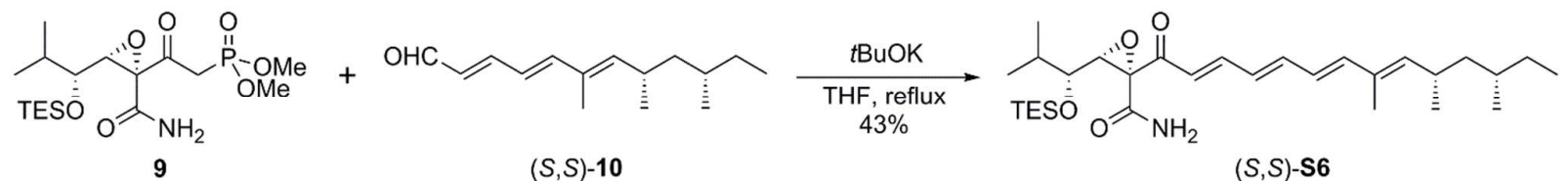

Amide $(S, S)$-S6 (100 mg, 43\% yield, yellow amorphous) was obtained from 9 (386 mg, $0.911 \mathrm{mmol}$ ) and $(S, S)-10(100 \mathrm{mg}, 0.454 \mathrm{mmol})$ by using a procedure similar to that for the $(R, R)$-isomer.

$[\alpha]_{\mathrm{D}}{ }^{20}+17.3^{\circ}\left(c 0.95, \mathrm{CHCl}_{3}\right)$; IR (neat) $3485,3346,2962,2877,1701,1548,1463,1352 \mathrm{~cm}^{-1} ;{ }^{1} \mathrm{H}$ NMR (400 MHz, $\left.\mathrm{CDCl}_{3}\right) \delta 7.49(\mathrm{br} d, J=3.0 \mathrm{~Hz}, 1 \mathrm{H}), 7.48(\mathrm{dd}, J=15.0,11.6 \mathrm{~Hz}, 1 \mathrm{H}), 6.74(\mathrm{dd}, J$ $=14.7,10.8 \mathrm{~Hz}, 1 \mathrm{H}), 6.53(\mathrm{~d}, J=15.0 \mathrm{~Hz}, 1 \mathrm{H}), 6.47(\mathrm{~d}, J=15.2 \mathrm{~Hz}, 1 \mathrm{H}), 6.35(\mathrm{dd}, J=14.7,11.6$ $\mathrm{Hz}, 1 \mathrm{H}), 6.23$ (dd, $J=15.2,10.8 \mathrm{~Hz}, 1 \mathrm{H}), 5.82$ (br d, $J=3.0 \mathrm{~Hz}, 1 \mathrm{H}), 5.42$ (d, $J=9.6 \mathrm{~Hz}, 1 \mathrm{H}), 3.48$ $(\mathrm{dd}, J=8.4,5.6 \mathrm{~Hz}, 1 \mathrm{H}), 3.14(\mathrm{~d}, J=8.4 \mathrm{~Hz}, 1 \mathrm{H}), 2.66-2.55(\mathrm{~m}, 1 \mathrm{H}), 1.78(\mathrm{~s}, 3 \mathrm{H}), 1.78-1.67(\mathrm{~m}$, $1 \mathrm{H}), 1.31-1.18(\mathrm{~m}, 3 \mathrm{H}), 1.14-1.05(\mathrm{~m}, 2 \mathrm{H}), 0.97(\mathrm{t}, J=8.0 \mathrm{~Hz}, 9 \mathrm{H}), 0.94(\mathrm{~d}, J=6.8 \mathrm{~Hz}, 3 \mathrm{H}), 0.89$ (d, $J=6.8 \mathrm{~Hz}, 3 \mathrm{H}), 0.87(\mathrm{~d}, J=6.8 \mathrm{~Hz}, 3 \mathrm{H}), 0.82(\mathrm{t}, J=7.2 \mathrm{~Hz}, 3 \mathrm{H}), 0.80(\mathrm{~d}, J=6.4 \mathrm{~Hz}, 3 \mathrm{H}), 0.75-0.62$ $(\mathrm{m}, 6 \mathrm{H}) ;{ }^{13} \mathrm{C} \mathrm{NMR}\left(100 \mathrm{MHz}, \mathrm{CDCl}_{3}\right) \delta 193.6,165.9,147.6,145.8,145.2,144.7,132.5,129.0$, $125.5,120.5,74.2,65.5,64.5,44.7,32.4,32.3,30.8,30.1,21.3,19.1,18.8,17.1,12.4,11.2,6.8$ (3C), 5.0 (3C); HRMS (EI+) $m / z$ calcd for $\mathrm{C}_{30} \mathrm{H}_{51} \mathrm{NO}_{4} \mathrm{Si}[\mathrm{M}]^{+}: 517.3587$ found 517.3582.

$(2 R, 3 S)-3-((R)-1-H y d r o x y-2-m e t h y l p r o p y l)-2-((2 E, 4 E, 6 E, 8 E, 10 R, 12 R)-8,10,12$-trimethyltetrade ca-2,4,6,8-tetraenoyl)oxirane-2-carboxamide $((R, R)-22)$
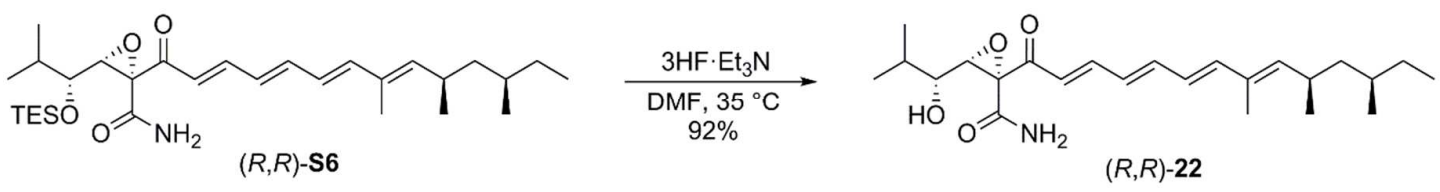

(All operations were performed in the dark.) To a stirred solution of tetraene amide $(R, R)$-S6 $(25 \mathrm{mg}$, $0.048 \mathrm{mmol})$ in DMF $(5.0 \mathrm{~mL})$ was added $3 \mathrm{HF} \cdot \mathrm{Et}_{3} \mathrm{~N}(0.03 \mathrm{~mL}, 0.18 \mathrm{mmol})$ at room temperature. After stirring at $35{ }^{\circ} \mathrm{C}$ for $1 \mathrm{~h}$, additional $3 \mathrm{HF} \cdot \mathrm{Et}_{3} \mathrm{~N}(0.03 \mathrm{~mL}, 0.18 \mathrm{mmol})$ was added. After $1 \mathrm{~h}$, the reaction was concentrated in vacuo. The crude residue was purified by flash chromatography on silica gel (1:1 hexane:EtOAc) to afford alcohol $(R, R)-\mathbf{2 2}(18 \mathrm{mg}, 92 \%$ yield) as a yellow amorphous. $[\alpha]_{\mathrm{D}}{ }^{20}-33.4^{\circ}\left(c 0.98, \mathrm{CHCl}_{3}\right)$; IR (neat) 3416, 3325, 2962, 2874, 1686, 1558, 1463, $1352 \mathrm{~cm}^{-1} ;{ }^{1} \mathrm{H}$ NMR (400 MHz, $\mathrm{CDCl}_{3}$ ) $\delta 7.72$ (br s, $\left.1 \mathrm{H}\right), 7.48$ (dd, $\left.J=15.2,11.6 \mathrm{~Hz}, 1 \mathrm{H}\right), 6.74(\mathrm{dd}, J=14.5,10.8$ $\mathrm{Hz}, 1 \mathrm{H}), 6.55$ (d, $J=15.2 \mathrm{~Hz}, 1 \mathrm{H}), 6.46$ (d, $J=15.2 \mathrm{~Hz}, 1 \mathrm{H}), 6.44$ (br s, 1H), 6.33 (dd, $J=14.5,11.6$ Hz, 1H), 6.22 (dd, $J=15.2,10.8 \mathrm{~Hz}, 1 \mathrm{H}), 5.41$ (d, $J=9.6 \mathrm{~Hz}, 1 \mathrm{H}), 3.58$ (br s, 1H), 3.39 (t, $J=7.8$ $\mathrm{Hz}, 1 \mathrm{H}), 3.18(\mathrm{~d}, J=7.8 \mathrm{~Hz}, 1 \mathrm{H}), 2.64-2.54(\mathrm{~m}, 1 \mathrm{H}), 1.79-1.73(\mathrm{~m}, 1 \mathrm{H}), 1.77$ (br s, 3H), 1.30-1.17 
(m, 3H), 1.12-1.04 (m, 2H), $0.97(\mathrm{~d}, J=6.8 \mathrm{~Hz}, 3 \mathrm{H}), 0.93(\mathrm{~d}, J=7.2 \mathrm{~Hz}, 3 \mathrm{H}), 0.90(\mathrm{~d}, J=6.8 \mathrm{~Hz}$, $3 \mathrm{H}), 0.81(\mathrm{t}, J=7.2 \mathrm{~Hz}, 3 \mathrm{H}), 0.79(\mathrm{~d}, J=6.0 \mathrm{~Hz}, 3 \mathrm{H}) ;{ }^{13} \mathrm{C} \mathrm{NMR}\left(100 \mathrm{MHz}, \mathrm{CDCl}_{3}\right) \delta 193.3,166.7$, 147.8, 146.1, 145.3, 144.9, 132.5, 129.0, 125.5, 120.4, 72.7, 65.3, 65.0, 44.6, 32.3, 31.8, 30.7, 30.0, 21.3, 19.0, 18.1, 18.0, 12.4, 11.2; HRMS (EI+) $\mathrm{m} / z$ calcd for $\mathrm{C}_{24} \mathrm{H}_{37} \mathrm{NO}_{4}[\mathrm{M}]^{+}: 403.2723$ found 403.2722 .

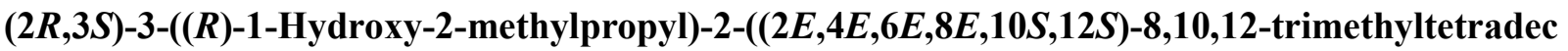
a-2,4,6,8-tetraenoyl)oxirane-2-carboxamide $((S, S)-22)$
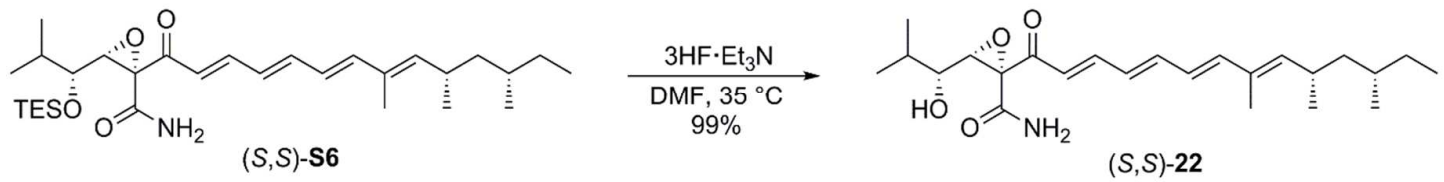

Alcohol $(S, S)$-22 (79 mg, 99\% yield, yellow amorphous) was obtained from $(S, S)$-S6 (100 mg, 0.193 mmol) by using a procedure similar to that for the $(R, R)$-isomer.

$[\alpha]_{\mathrm{D}}{ }^{20}+9.6^{\circ}$ (c 0.68, $\mathrm{CHCl}_{3}$ ); IR (neat) 3412, 3320, 2961, 2874, 1683, 1557, 1463, $1351 \mathrm{~cm}^{-1} ;{ }^{1} \mathrm{H}$ NMR (400 MHz, $\left.\mathrm{CDCl}_{3}\right) \delta 7.76(\mathrm{br} \mathrm{d}, J=2.6 \mathrm{~Hz}, 1 \mathrm{H}), 7.58(\mathrm{dd}, J=15.1,11.6 \mathrm{~Hz}, 1 \mathrm{H}), 6.78(\mathrm{dd}, J$ $=14.6,11.2 \mathrm{~Hz}, 1 \mathrm{H}), 6.57(\mathrm{~d}, J=15.0 \mathrm{~Hz}, 1 \mathrm{H}), 6.50(\mathrm{~d}, J=15.1 \mathrm{~Hz}, 1 \mathrm{H}), 6.37(\mathrm{dd}, J=14.6,11.6 \mathrm{~Hz}$, 1H), 6.26 (dd, $J=15.0,11.2 \mathrm{~Hz}, 1 \mathrm{H}), 6.15(\mathrm{br} \mathrm{d}, J=2.6 \mathrm{~Hz}, 1 \mathrm{H}), 5.45$ (d, $J=10.0 \mathrm{~Hz}, 1 \mathrm{H}), 3.43$ (t, $J$ $=7.6 \mathrm{~Hz}, 1 \mathrm{H}), 3.20(\mathrm{~d}, J=7.6 \mathrm{~Hz}, 1 \mathrm{H}), 3.16(\mathrm{br} \mathrm{s}, 1 \mathrm{H}), 2.69-2.58(\mathrm{~m}, 1 \mathrm{H}), 1.85-1.75(\mathrm{~m}, 1 \mathrm{H}), 1.81$ (br s, 3H), 1.34-1.20 (m, 3H), 1.11-1.08 (m, 2H), $1.01(\mathrm{~d}, J=6.8 \mathrm{~Hz}, 3 \mathrm{H}), 0.96(\mathrm{~d}, J=6.4 \mathrm{~Hz}, 3 \mathrm{H})$, $0.93(\mathrm{~d}, J=6.8 \mathrm{~Hz}, 3 \mathrm{H}), 0.84(\mathrm{t}, J=7.2 \mathrm{~Hz}, 3 \mathrm{H}), 0.82(\mathrm{~d}, J=6.0 \mathrm{~Hz}, 3 \mathrm{H}) ;{ }^{13} \mathrm{C} \mathrm{NMR}(100 \mathrm{MHz}$, $\left.\mathrm{CDCl}_{3}\right) \delta 193.5,166.7,148.0,146.2,145.4,145.0,132.5,128.9,125.5,120.2,72.8,65.2,64.9,44.7$, $32.3,31.9,30.8,30.1,21.3,19.1,18.1,17.9,12.4,11.2$; HRMS (EI+) $m / z$ calcd for $\mathrm{C}_{24} \mathrm{H}_{37} \mathrm{NO}_{4}[\mathrm{M}]^{+}$: 403.2723 found 403.2717 .

$(1 R, 4 R, 5 R)-4-H y d r o x y-4-i s o p r o p y l-1-((2 E, 4 E, 6 E, 8 E, 10 R, 12 R)-8,10,12-t r i m e t h y l t e t r a d e c a-2,4,6$, 8-tetraenoyl)-6-oxa-3-azabicyclo[3.1.0]hexan-2-one (2) and $(1 R, 4 S, 5 R)-4-H y d r o x y-4$-isopropyl-1-((2E,4E,6E,8E,10R,12R)-8,10,12-trimethyltetradeca-2,4,6, 8-tetraenoyl)-6-oxa-3-azabicyclo[3.1.0]hexan-2-one (3) 

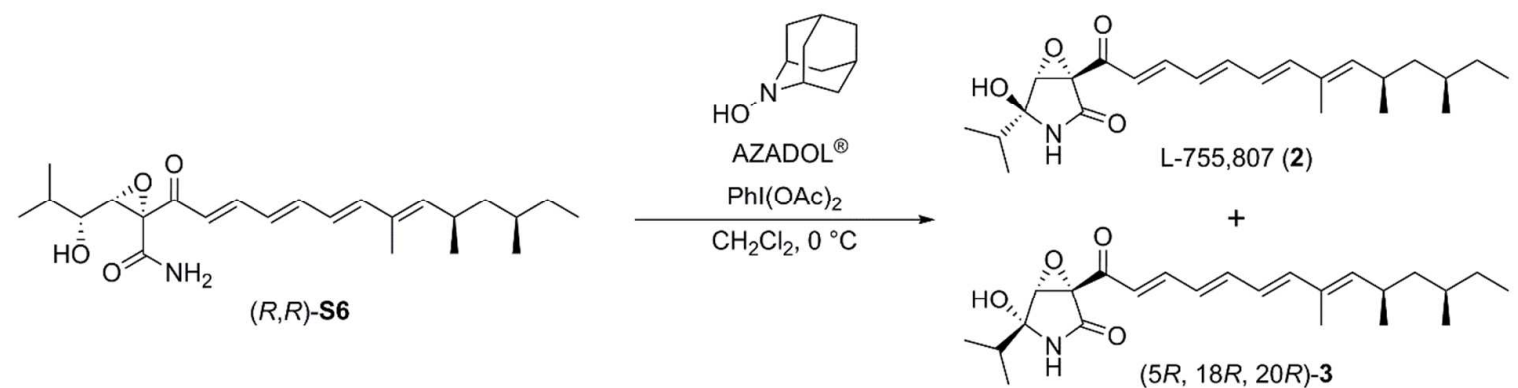

(All operations were performed in the dark.) To a stirred solution of alcohol $(R, R)-\mathbf{S 6}$ (40 $\mathrm{mg}, 0.099$ $\mathrm{mmol})$ in $\mathrm{CH}_{2} \mathrm{Cl}_{2}(1.0 \mathrm{~mL})$ was successively added $\mathrm{AZADOL}{ }^{\circledR}(3.2 \mathrm{mg}, 0.093 \mathrm{mmol})$ and $\mathrm{PhI}(\mathrm{OAc})_{2}$ (41 $\mathrm{mg}, 0.13 \mathrm{mmol}$ ) at $0{ }^{\circ} \mathrm{C}$. The reaction mixture was stirred for $12 \mathrm{~h}$. The reaction was quenched by addition of saturated aqueous $\mathrm{NaHCO}_{3}$ and $\mathrm{Na}_{2} \mathrm{~S}_{2} \mathrm{O}_{3}$ solution and stirred vigorously for $10 \mathrm{~min}$. The mixture was extracted with $\mathrm{CH}_{2} \mathrm{Cl}_{2}$ ( 3 times). The combined organic layers were dried over $\mathrm{Na}_{2} \mathrm{SO}_{4}$, filtered, and concentrated in vacuo. The crude residue was purified by flash chromatography on silica gel (4:1 hexane:acetone) to afford a 3:1 mixture of 2 and $\mathbf{3}$ (34.6 $\mathrm{mg}, 87 \%$ yield) as a yellow amorphous. Compounds $2(25.2 \mathrm{mg}, 63 \%$ yield, yellow amorphous) and $3(8.4 \mathrm{mg}, 21 \%$ yield, yellow amorphous) were obtained after repeated column chromatography.

2: $[\alpha]_{\mathrm{D}}{ }^{20}-96.1^{\circ}$ (c 0.65, MeOH); IR (neat) 3454, 3231, 2962, 2925, 1732, 1708, 1659, 1577, 1407 $\mathrm{cm}^{-1} ;{ }^{1} \mathrm{H}$ NMR (400 MHz, $\left.\mathrm{CD}_{2} \mathrm{Cl}_{2}\right) \delta 7.87(\mathrm{br} \mathrm{s}, 1 \mathrm{H}), 7.33(\mathrm{dd}, J=15.1,11.6 \mathrm{~Hz}, 1 \mathrm{H}), 6.71(\mathrm{dd}, J=$ 14.6, $10.6 \mathrm{~Hz}, 1 \mathrm{H}), 6.48(\mathrm{~d}, J=15.1 \mathrm{~Hz}, 1 \mathrm{H}), 6.31$ (d, $J=15.1 \mathrm{~Hz}, 1 \mathrm{H}), 6.31$ (dd, $J=14.6,11.6 \mathrm{~Hz}$, $1 \mathrm{H}), 6.23(\mathrm{dd}, J=15.1,10.6 \mathrm{~Hz}, 1 \mathrm{H}), 5.45(\mathrm{~d}, J=9.2 \mathrm{~Hz}, 1 \mathrm{H}), 4.49(\mathrm{br} \mathrm{s}, 1 \mathrm{H}), 4.25(\mathrm{~d}, J=2.8 \mathrm{~Hz}$, $1 \mathrm{H}), 2.68-2.60(\mathrm{~m}, 1 \mathrm{H}), 2.08-2.01(\mathrm{~m}, 1 \mathrm{H}), 1.77(\mathrm{br} \mathrm{s}, 3 \mathrm{H}), 1.34-1.27(\mathrm{~m}, 2 \mathrm{H}), 1.31-1.22(\mathrm{~m}, 1 \mathrm{H})$, $1.18-1.03$ (m, 2H), 1.15 (d, $J=6.8 \mathrm{~Hz}, 3 \mathrm{H}), 1.09$ (d, $J=6.8 \mathrm{~Hz}, 3 \mathrm{H}), 0.97$ (d, $J=6.8 \mathrm{~Hz}, 3 \mathrm{H}), 0.85$ (t, $J=7.2 \mathrm{~Hz}, 3 \mathrm{H}$ ), $0.83(\mathrm{~d}, J=6.4 \mathrm{~Hz}, 3 \mathrm{H}) ;{ }^{13} \mathrm{C}$ NMR $\left(100 \mathrm{MHz}, \mathrm{CD}_{2} \mathrm{Cl}_{2}\right) \delta 189.9,170.2,146.7$, 146.2, 145.4, 144.8, 133.0, 129.1, 125.9, 120.9, 87.7, 65.9, 61.0, 45.0, 33.7, 32.7, 31.1, 30.4, 21.4, 19.3, 17.8, 16.3, 12.6, 11.4; HRMS (EI+) $m / z$ calcd for $\mathrm{C}_{24} \mathrm{H}_{35} \mathrm{NO}_{4}[\mathrm{M}]^{+}: 401.2566$ found 401.2567. 3: $[\alpha]_{\mathrm{D}}{ }^{20}-39.0^{\circ}$ (c 0.67, MeOH); IR (neat) 3305, 2963, 2924, 2875, 1726, 1577, 1560, 1153, 1096, $1006 \mathrm{~cm}^{-1} ;{ }^{1} \mathrm{H}$ NMR $\left(400 \mathrm{MHz}, \mathrm{CD}_{2} \mathrm{Cl}_{2}\right) \delta 7.50(\mathrm{dd}, J=15.0,11.6 \mathrm{~Hz}, 1 \mathrm{H}), 6.82(\mathrm{dd}, J=14.8,10.8$ $\mathrm{Hz}, 1 \mathrm{H}), 6.53(\mathrm{~d}, J=15.0 \mathrm{~Hz}, 1 \mathrm{H}), 6.47$ (d, $J=15.2 \mathrm{~Hz}, 1 \mathrm{H}), 6.39$ (dd, $J=14.8,11.6 \mathrm{~Hz}, 1 \mathrm{H}), 6.30$ (dd, $J=15.2,10.8 \mathrm{~Hz}, 1 \mathrm{H}), 6.16$ (br s, 1H), 5.47 (d, $J=10.0 \mathrm{~Hz}, 1 \mathrm{H}$ ), 3.98 (d, $J=2.8 \mathrm{~Hz}, 1 \mathrm{H}$ ), 3.26 (br s, $1 \mathrm{H}), 2.70-2.61(\mathrm{~m}, 1 \mathrm{H}), 2.12-2.06(\mathrm{~m}, 1 \mathrm{H}), 1.81(\mathrm{~d}, J=0.8 \mathrm{~Hz}, 3 \mathrm{H}), 1.35-1.21(\mathrm{~m}, 3 \mathrm{H})$, 1.19-1.09 (m, 2H), 1.04 (d, $J=6.8 \mathrm{~Hz}, 3 \mathrm{H}), 1.00$ (d, $J=7.2 \mathrm{~Hz}, 3 \mathrm{H}), 0.96$ (d, $J=6.4 \mathrm{~Hz}, 3 \mathrm{H}), 0.85$ (t, $J=7.2 \mathrm{~Hz}, 3 \mathrm{H}), 0.83(\mathrm{~d}, J=6.4 \mathrm{~Hz}, 3 \mathrm{H}) ;{ }^{13} \mathrm{C} \mathrm{NMR}\left(100 \mathrm{MHz}, \mathrm{CD}_{2} \mathrm{Cl}_{2}\right) \delta 187.5,167.9,146.7,146.3$, 145.6, 145.1, 133.0, 129.2, 125.9, 122.4, 87.4, 63.5, 63.2, 45.0, 34.6, 32.8, 31.2, 30.5, 21.4, 19.2, 16.2, 16.0, 12.6, 11.4; HRMS (EI+) $m / z$ calcd for $\mathrm{C}_{24} \mathrm{H}_{35} \mathrm{NO}_{4}[\mathrm{M}]^{+}: 401.2566$ found 401.2565 . 
$(1 R, 4 R, 5 R)-4-H y d r o x y-4-i s o p r o p y l-1-((2 E, 4 E, 6 E, 8 E, 10 S, 12 S)-8,10,12-t r i m e t h y l t e t r a d e c a-2,4,6$, 8-tetraenoyl)-6-oxa-3-azabicyclo[3.1.0]hexan-2-one (4)

$(1 R, 4 S, 5 R)-4-H y d r o x y-4-i s o p r o p y l-1-((2 E, 4 E, 6 E, 8 E, 10 S, 12 S)-8,10,12-t r i m e t h y l t e t r a d e c a-2,4,6,8$ -tetraenoyl)-6-oxa-3-azabicyclo[3.1.0]hexan-2-one (5)

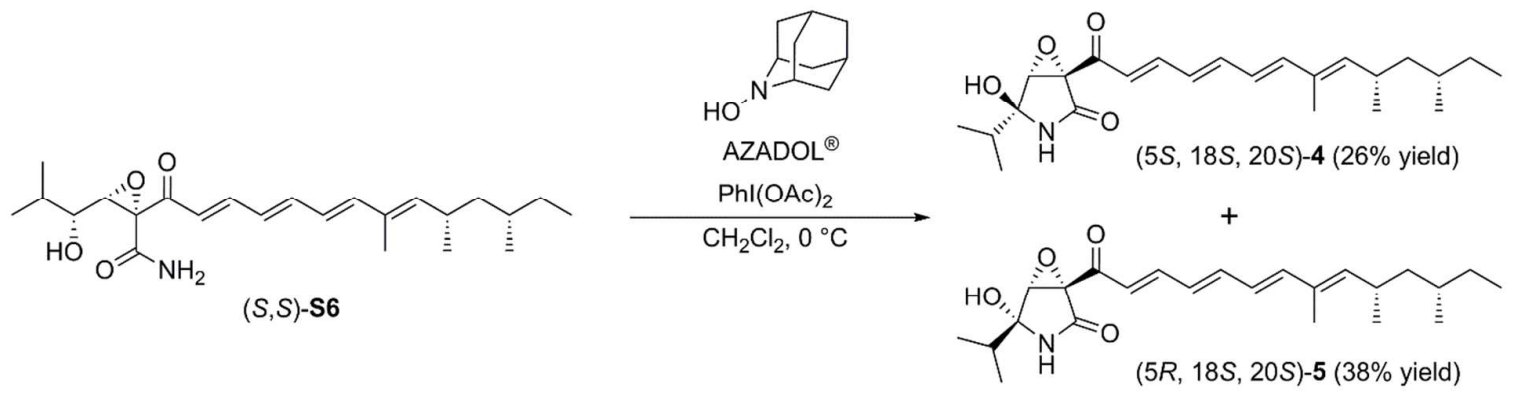

Compounds 4 (9.4 mg, 26\% yield, yellow amorphous) and 5 (13.4 mg, 38\% yield, yellow amorphous) were obtained from $(S, S)$-S6 $(35.7 \mathrm{mg}, 0.088 \mathrm{mmol})$ by using a procedure similar to that for the synthesis of compounds $\mathbf{2}$ and $\mathbf{3}$.

4: $[\alpha]_{\mathrm{D}}{ }^{20}-15.6^{\circ}$ (c 0.71, MeOH); IR (neat) 3454, 3235, 2962, 2924, 1732, 1707, 1658, 1578, 1407 $\mathrm{cm}^{-1}$; ${ }^{1} \mathrm{H}$ NMR (400 MHz, $\left.\mathrm{CD}_{2} \mathrm{Cl}_{2}\right) \delta 8.28$ (br s, $\left.1 \mathrm{H}\right), 7.24$ (dd, $\left.J=14.9,11.4 \mathrm{~Hz}, 1 \mathrm{H}\right), 6.68(\mathrm{dd}, J=$ 14.7, $11.0 \mathrm{~Hz}, 1 \mathrm{H}), 6.43$ (d, $J=14.9 \mathrm{~Hz}, 1 \mathrm{H}), 6.29$ (dd, $J=14.7,11.4 \mathrm{~Hz}, 1 \mathrm{H}), 6.27$ (d, $J=15.1 \mathrm{~Hz}$, 1H), $6.21(\mathrm{dd}, J=15.1,11.0 \mathrm{~Hz}, 1 \mathrm{H}), 5.41$ (d, $J=9.6 \mathrm{~Hz}, 1 \mathrm{H}), 4.64$ (br s, 1H), 4.30 (d, $J=2.8 \mathrm{~Hz}$, $1 \mathrm{H}), 2.67-2.59(\mathrm{~m}, 1 \mathrm{H}), 2.07-2.00(\mathrm{~m}, 1 \mathrm{H}), 1.76$ (br s, 3H), 1.33-1.23 (m, 2H), 1.29-1.22 (m, 1H), 1.20-1.07 (m, 2H), $1.15(\mathrm{~d}, J=6.8 \mathrm{~Hz}, 3 \mathrm{H}), 1.11(\mathrm{~d}, J=6.8 \mathrm{~Hz}, 3 \mathrm{H}), 0.95(\mathrm{~d}, J=6.8 \mathrm{~Hz}, 3 \mathrm{H}), 0.84$ (t, $J=6.8 \mathrm{~Hz}, 3 \mathrm{H}), 0.82(\mathrm{~d}, J=6.0 \mathrm{~Hz}, 3 \mathrm{H}) ;{ }^{13} \mathrm{C} \mathrm{NMR}\left(100 \mathrm{MHz}, \mathrm{CD}_{2} \mathrm{Cl}_{2}\right) \delta 190.0,170.1,146.8,146.5$, $145.3,145.0,133.1,129.1,125.9,120.7,87.7,66.0,60.9,45.1,33.7,32.8,31.2,30.5,21.5,19.3$, 17.8, 16.3, 12.7, 11.5; HRMS (EI+) $m / z$ calcd for $\mathrm{C}_{24} \mathrm{H}_{35} \mathrm{NO}_{4}[\mathrm{M}]^{+}: 401.2566$ found 401.2562.

5: $[\alpha]_{\mathrm{D}}{ }^{24}-10.7^{\circ}(c$ 0.65, MeOH); IR (neat) 3422, 2963, 2926, 2875, 1729, 1577, 1560, 1153, 1097 , $1008 \mathrm{~cm}^{-1} ;{ }^{1} \mathrm{H}$ NMR $\left(400 \mathrm{MHz}, \mathrm{CD}_{2} \mathrm{Cl}_{2}\right) \delta 7.50(\mathrm{dd}, J=15.0,11.6 \mathrm{~Hz}, 1 \mathrm{H}), 6.82(\mathrm{dd}, J=14.8,10.8$ $\mathrm{Hz}, 1 \mathrm{H}), 6.53(\mathrm{~d}, J=15.0 \mathrm{~Hz}, 1 \mathrm{H}), 6.47$ (d, $J=15.2 \mathrm{~Hz}, 1 \mathrm{H}), 6.39$ (dd, $J=14.8,11.6 \mathrm{~Hz}, 1 \mathrm{H}), 6.30$ (dd, $J=15.2,10.8 \mathrm{~Hz}, 1 \mathrm{H}), 5.94$ (br s, 1H), 5.47 (d, $J=10.0 \mathrm{~Hz}, 1 \mathrm{H}), 3.99$ (d, $J=2.4 \mathrm{~Hz}, 1 \mathrm{H}), 3.09$ (br s, $1 \mathrm{H}), 2.71-2.59(\mathrm{~m}, 1 \mathrm{H}), 2.14-2.04(\mathrm{~m}, 1 \mathrm{H}), 1.81(\mathrm{~d}, J=1.2 \mathrm{~Hz}, 3 \mathrm{H}), 1.36-1.20(\mathrm{~m}, 3 \mathrm{H})$, 1.19-1.09 (m, 2H), $1.04(\mathrm{~d}, J=6.8 \mathrm{~Hz}, 3 \mathrm{H}), 1.00(\mathrm{~d}, J=6.8 \mathrm{~Hz}, 3 \mathrm{H}), 0.96(\mathrm{~d}, J=6.8 \mathrm{~Hz}, 3 \mathrm{H}), 0.85$ (t, $J=6.8 \mathrm{~Hz}, 3 \mathrm{H}), 0.83(\mathrm{~d}, J=6.4 \mathrm{~Hz}, 3 \mathrm{H}) ;{ }^{13} \mathrm{C} \mathrm{NMR}\left(100 \mathrm{MHz}, \mathrm{CD}_{2} \mathrm{Cl}_{2}\right) \delta 187.5,167.7,146.7,146.4$, $145.7,145.1,133.0,129.2,125.9,122.4,87.3,63.4,63.2,45.0,34.6,32.8,31.2,30.5,21.4$, 19.2, 16.2, 16.0, 12.6, 11.4; HRMS (EI+) $m / z$ calcd for $\mathrm{C}_{24} \mathrm{H}_{35} \mathrm{NO}_{4}[\mathrm{M}]^{+}: 401.2566$ found 401.2568. 


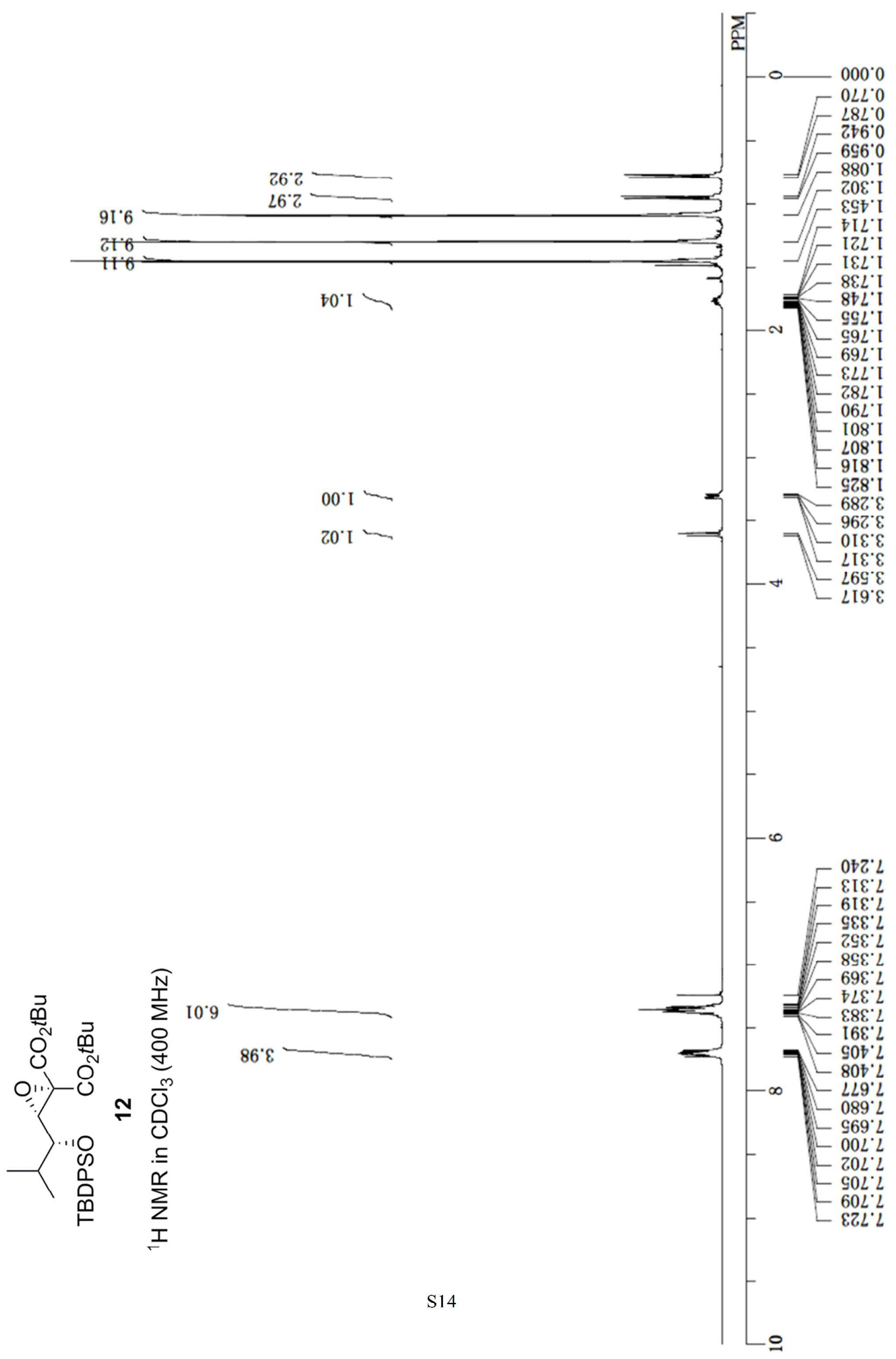




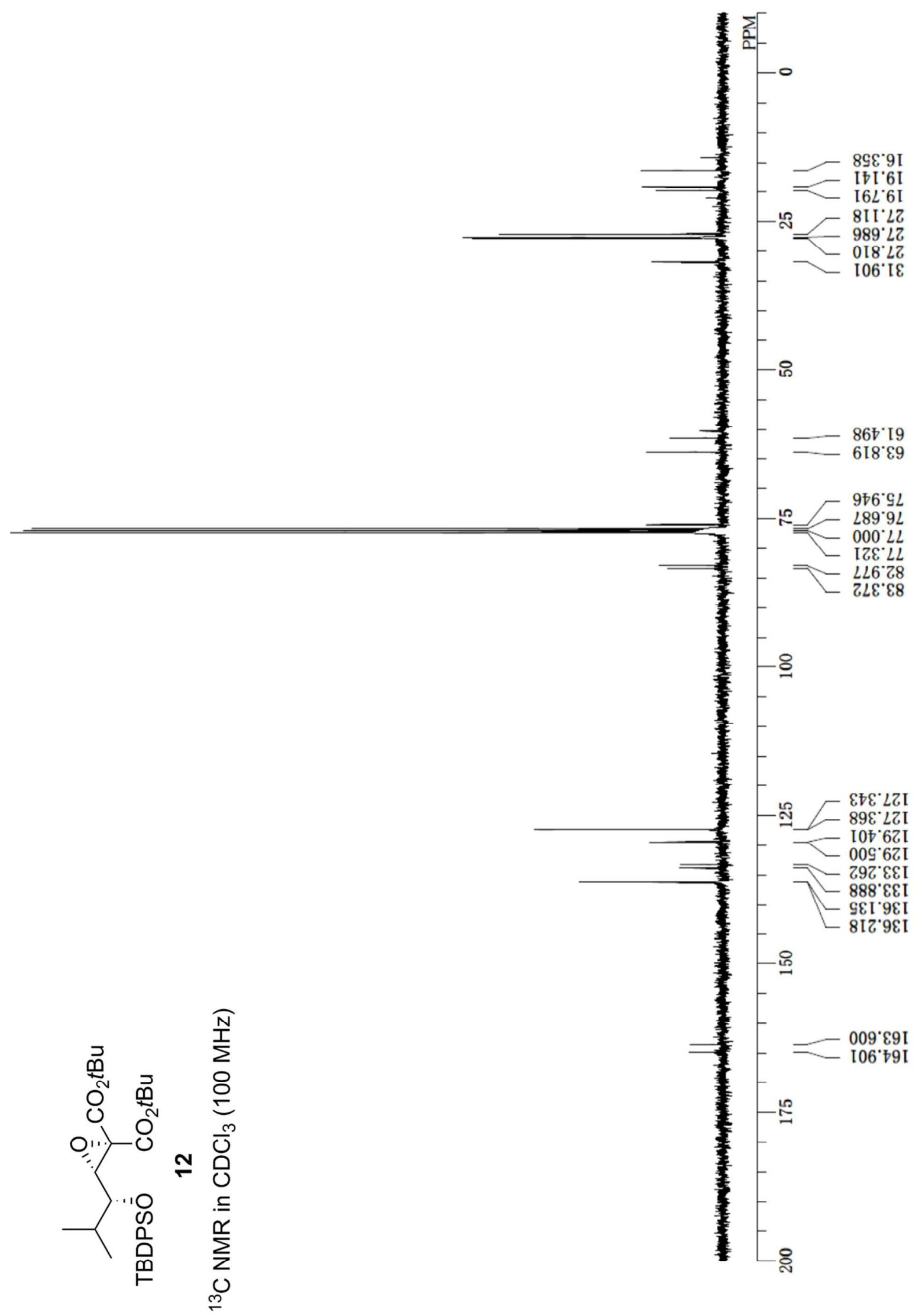




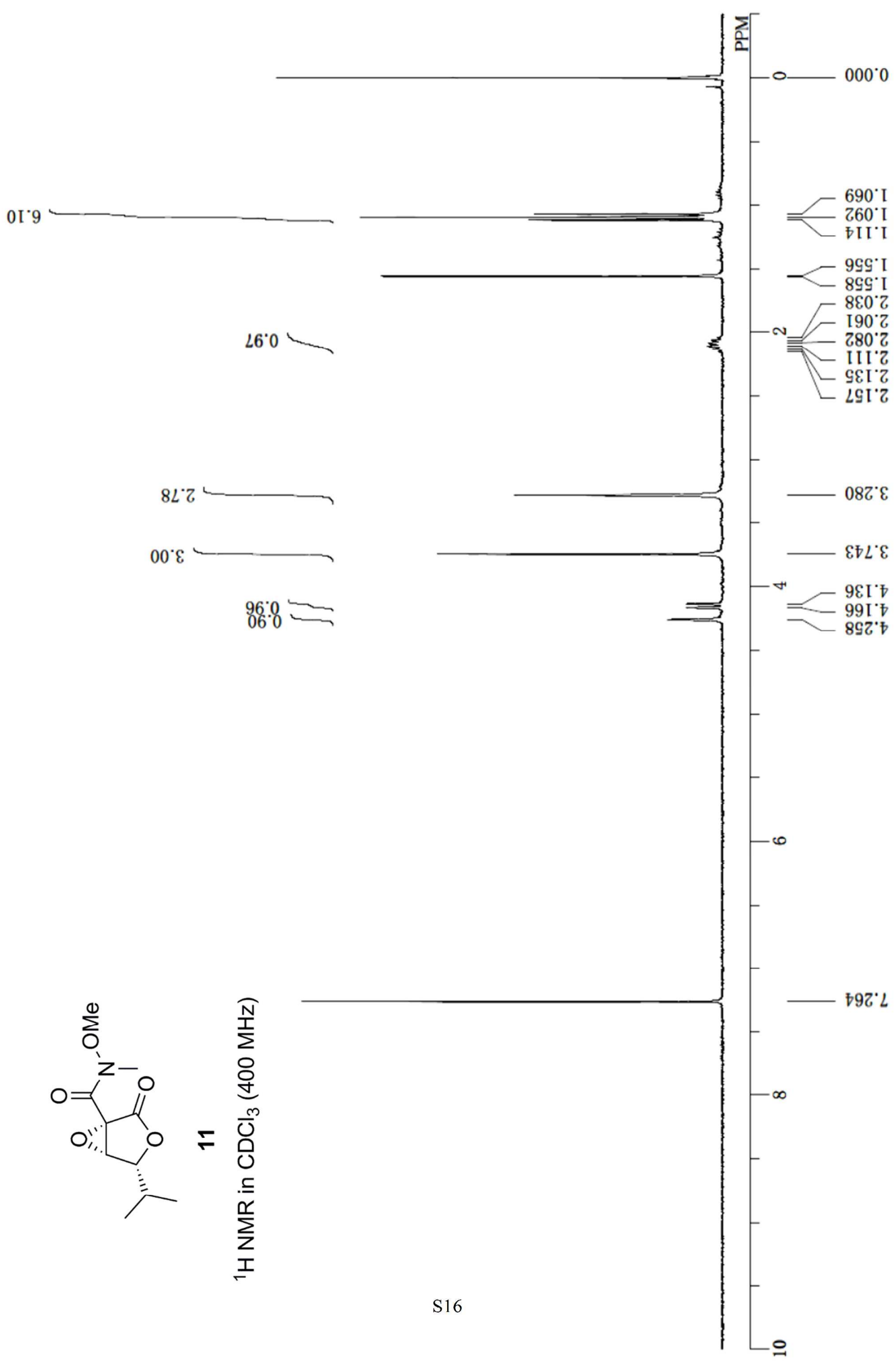




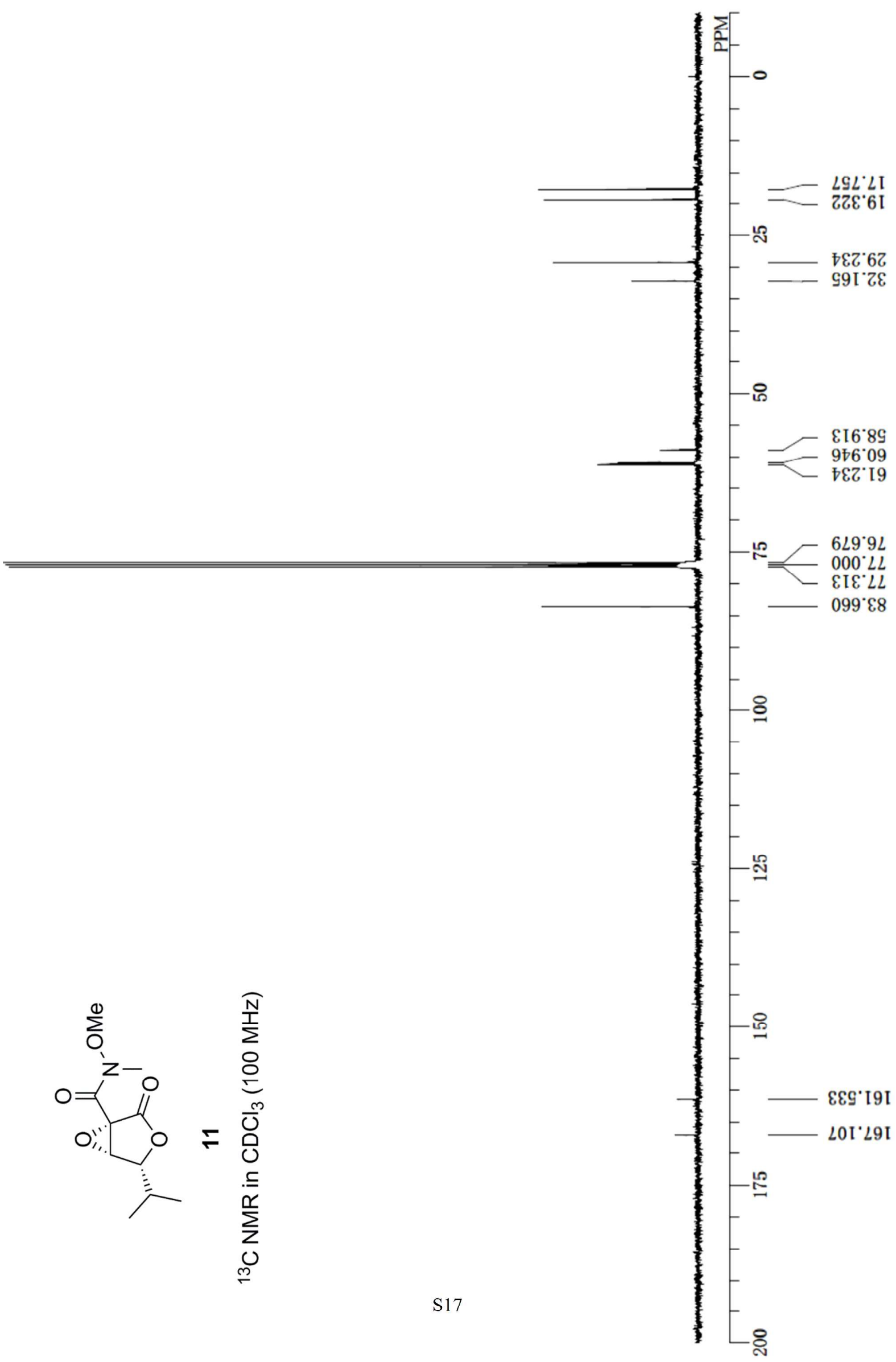




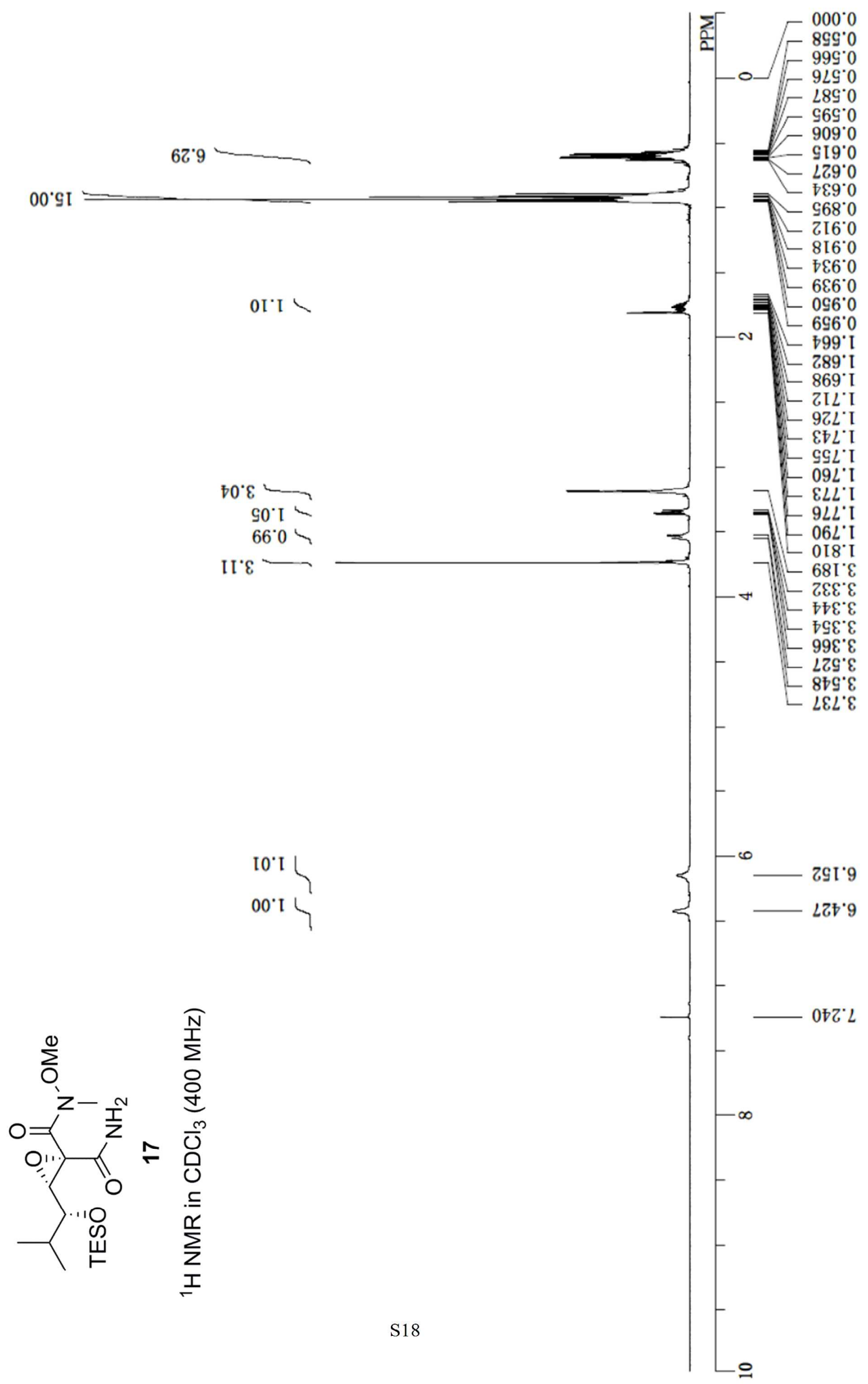




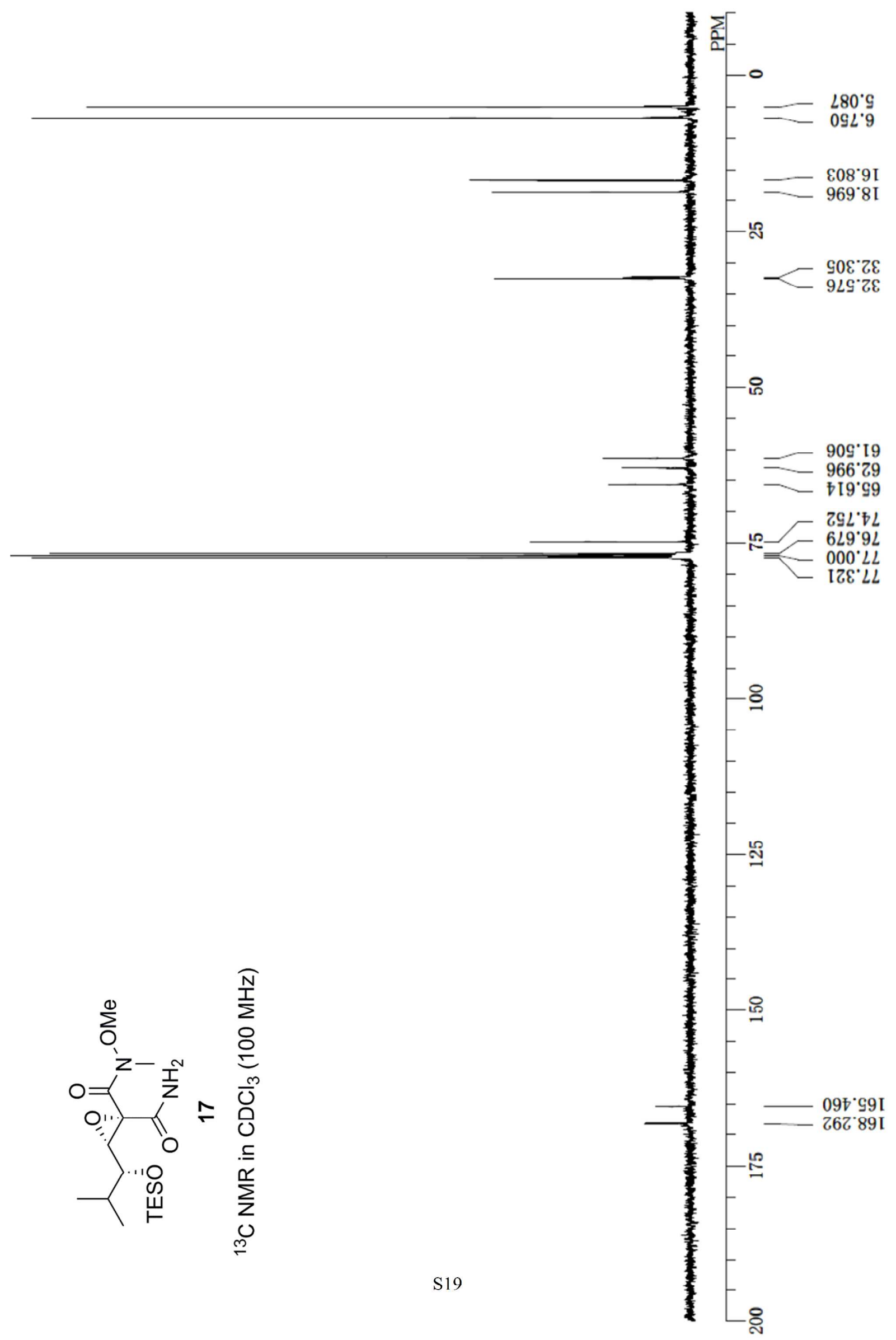




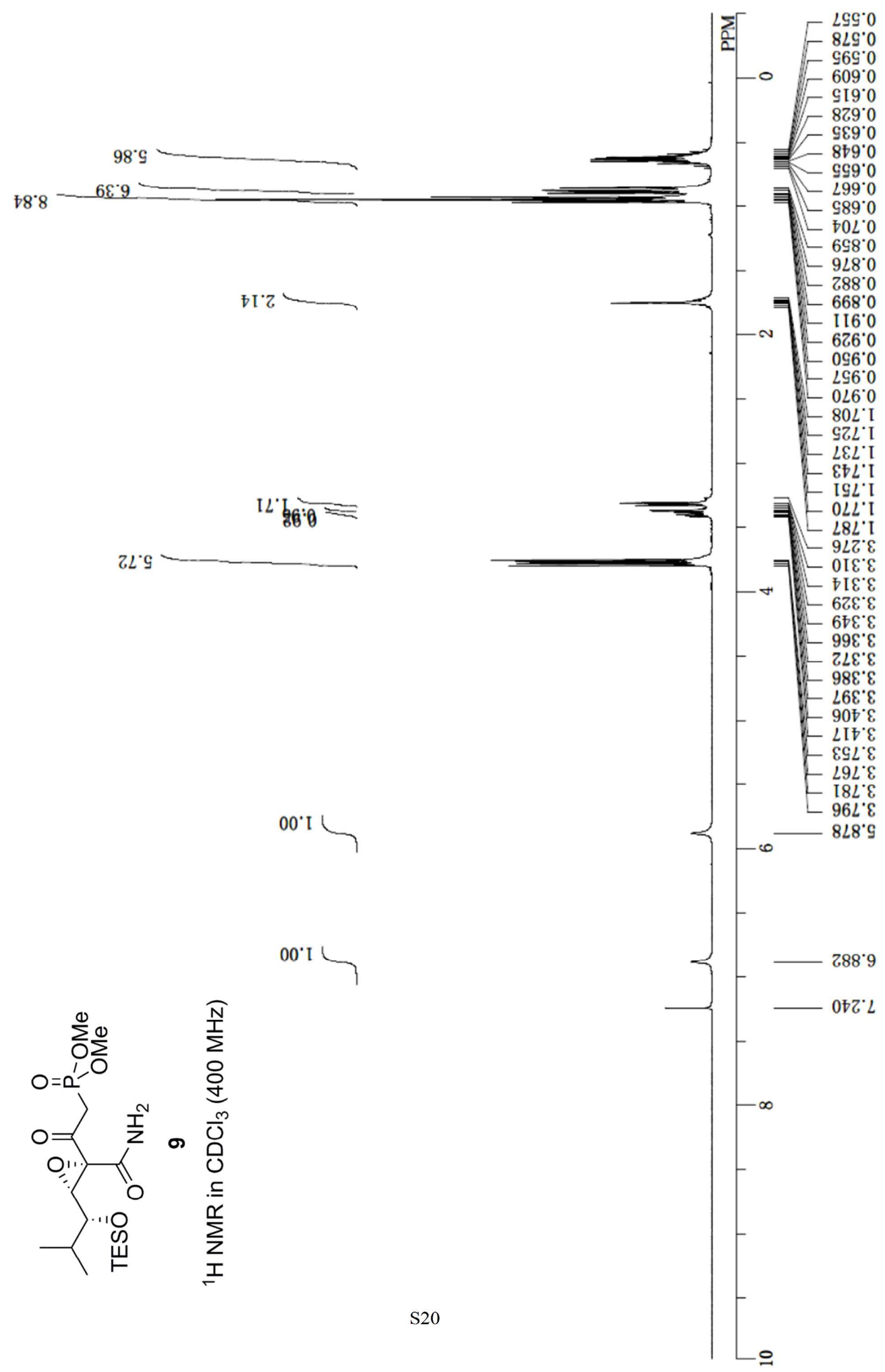




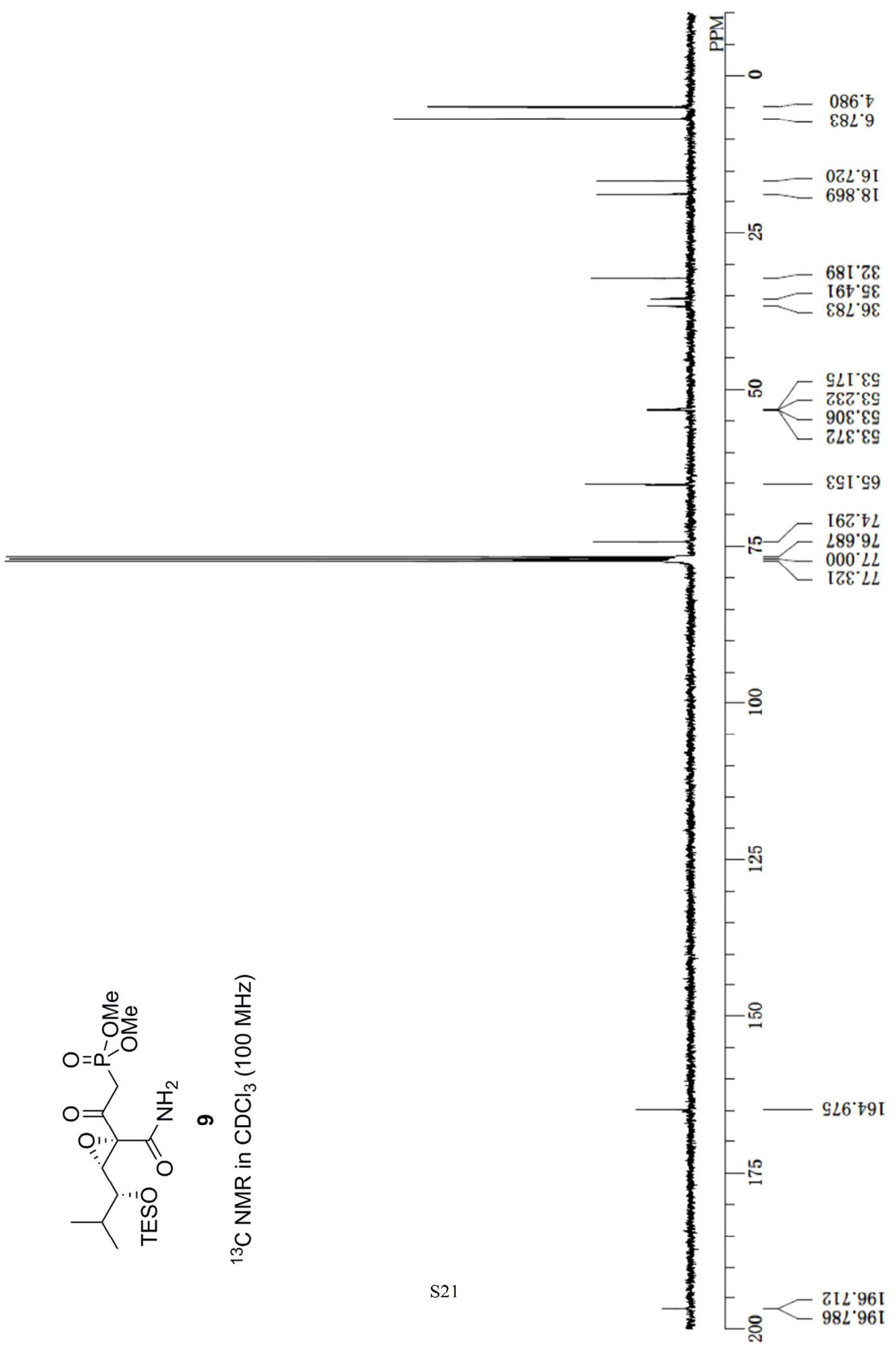



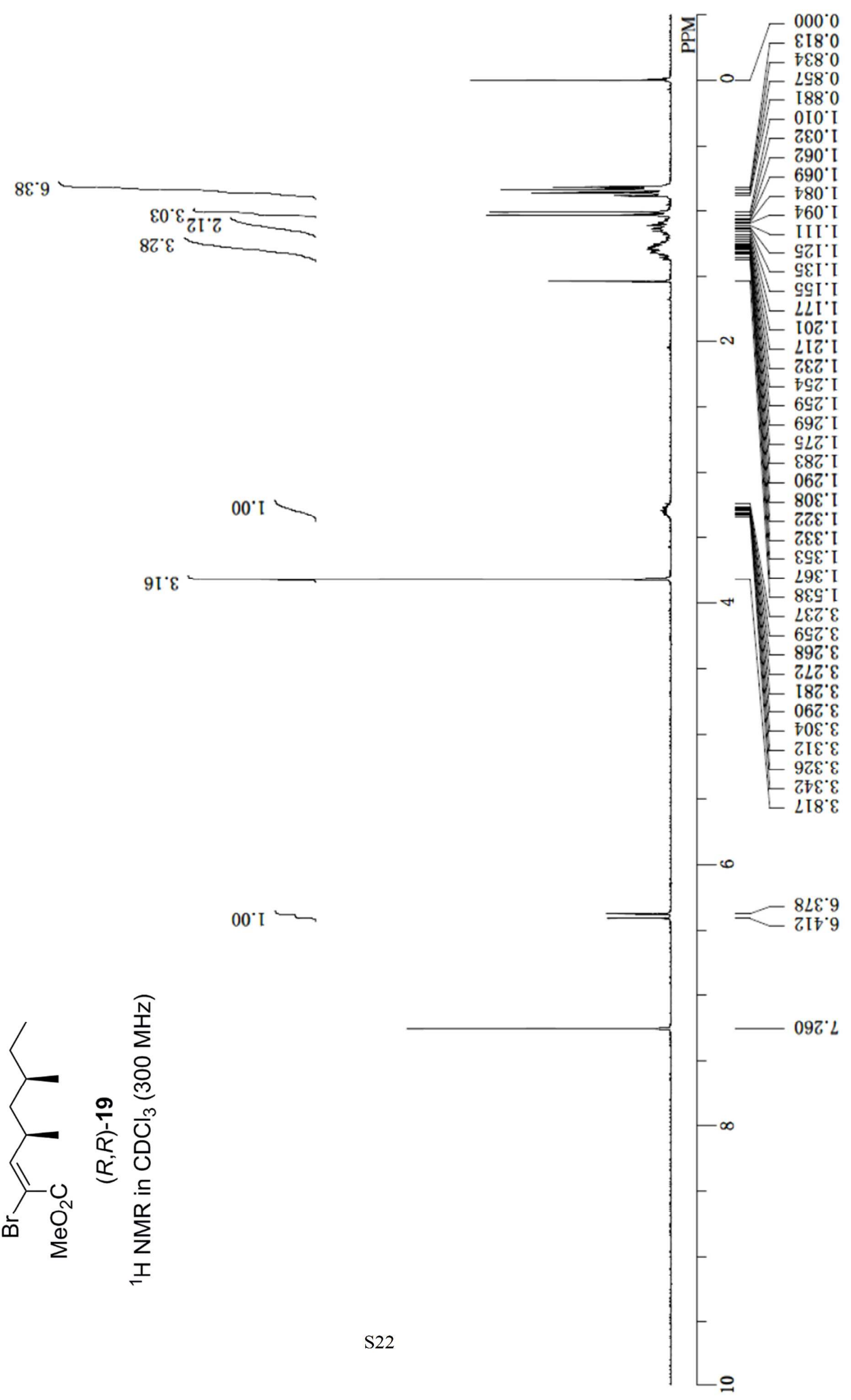


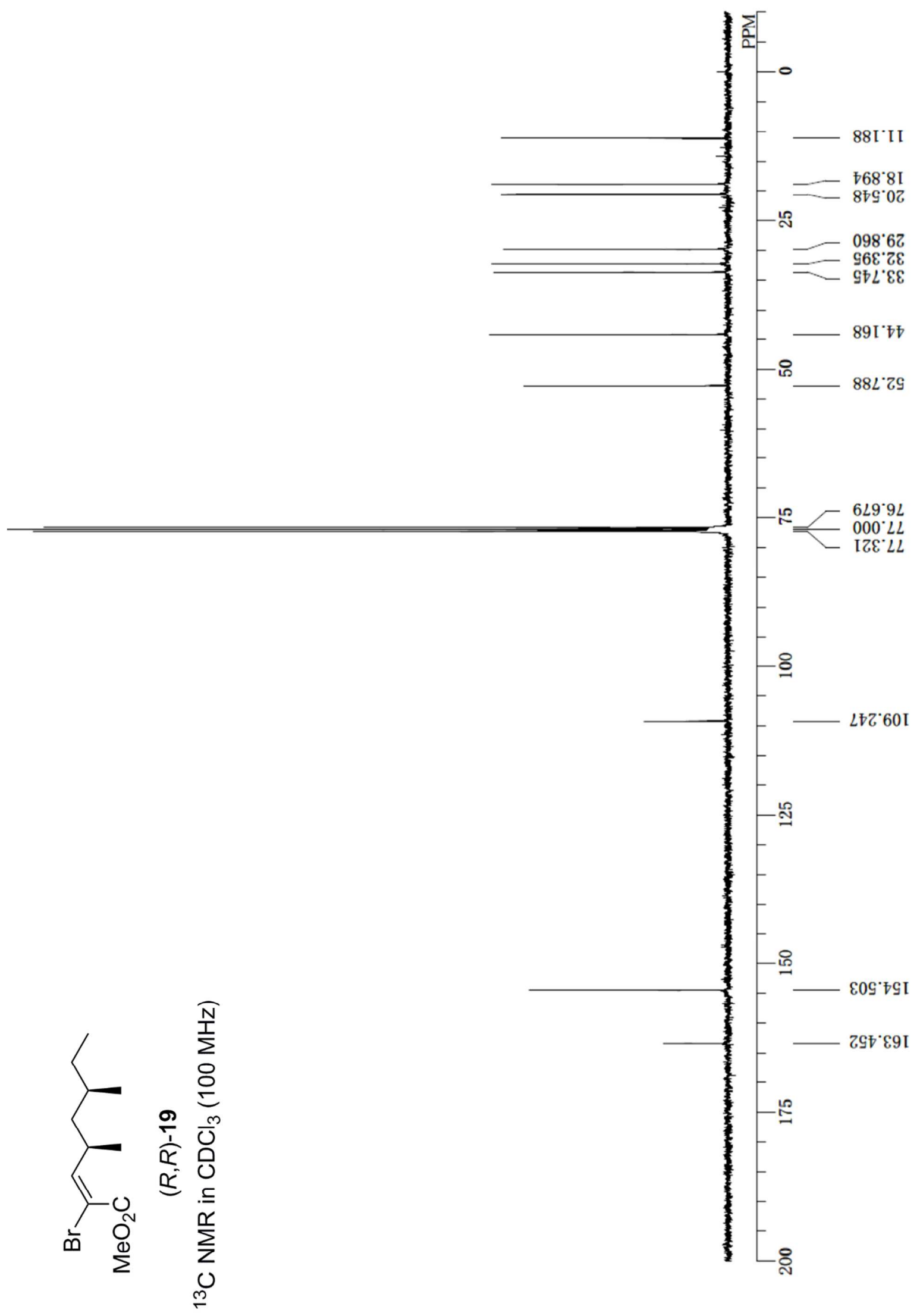




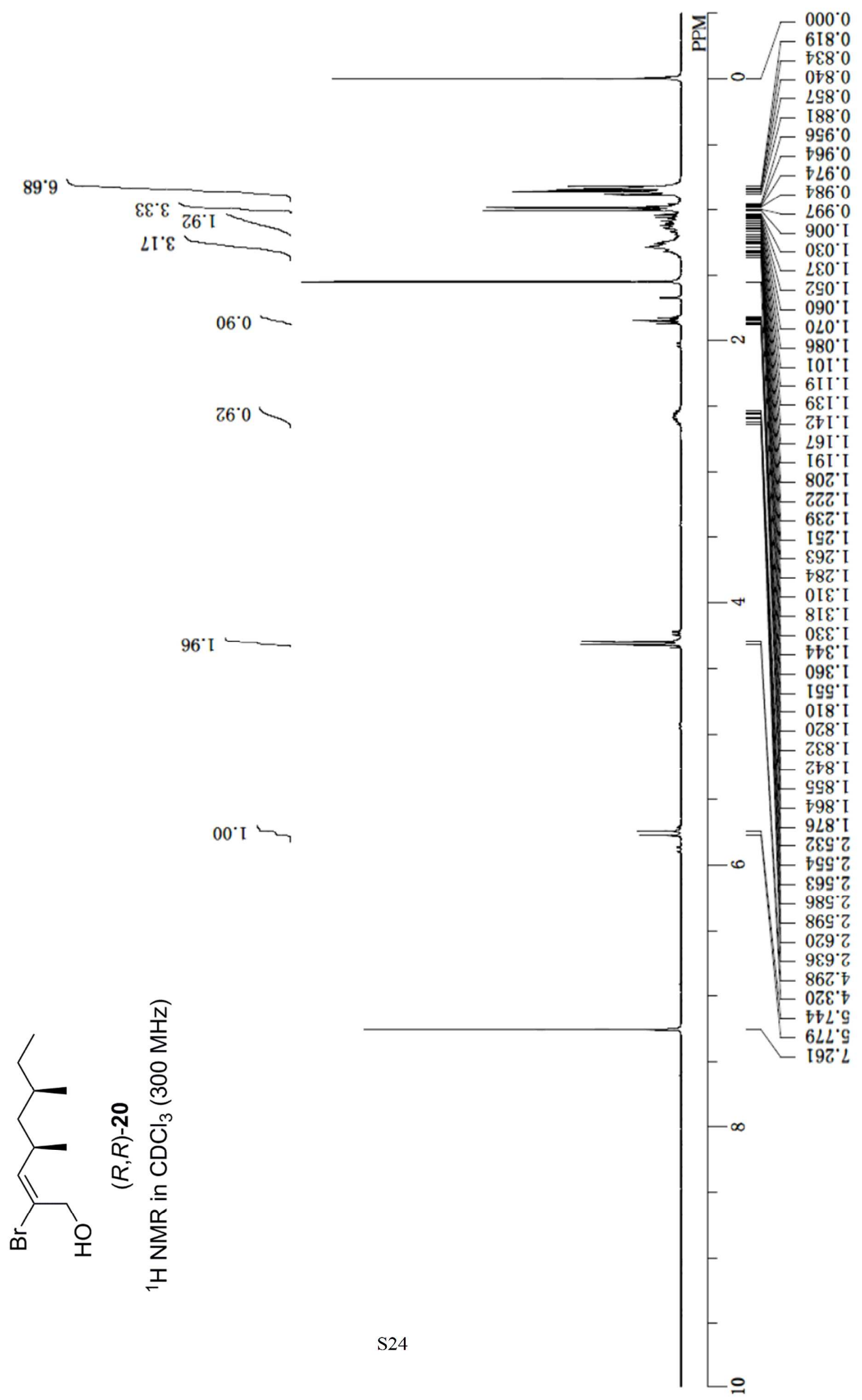




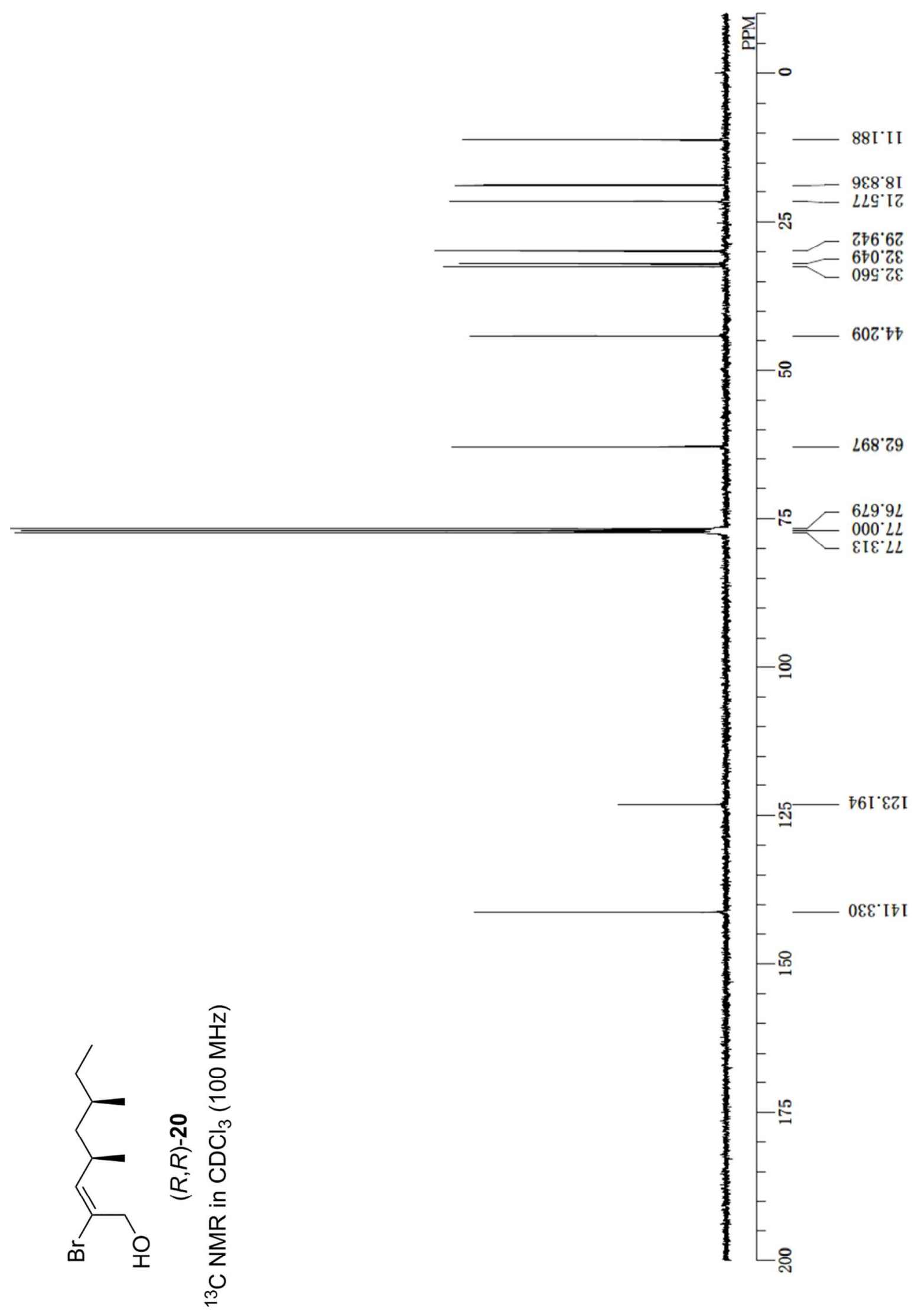




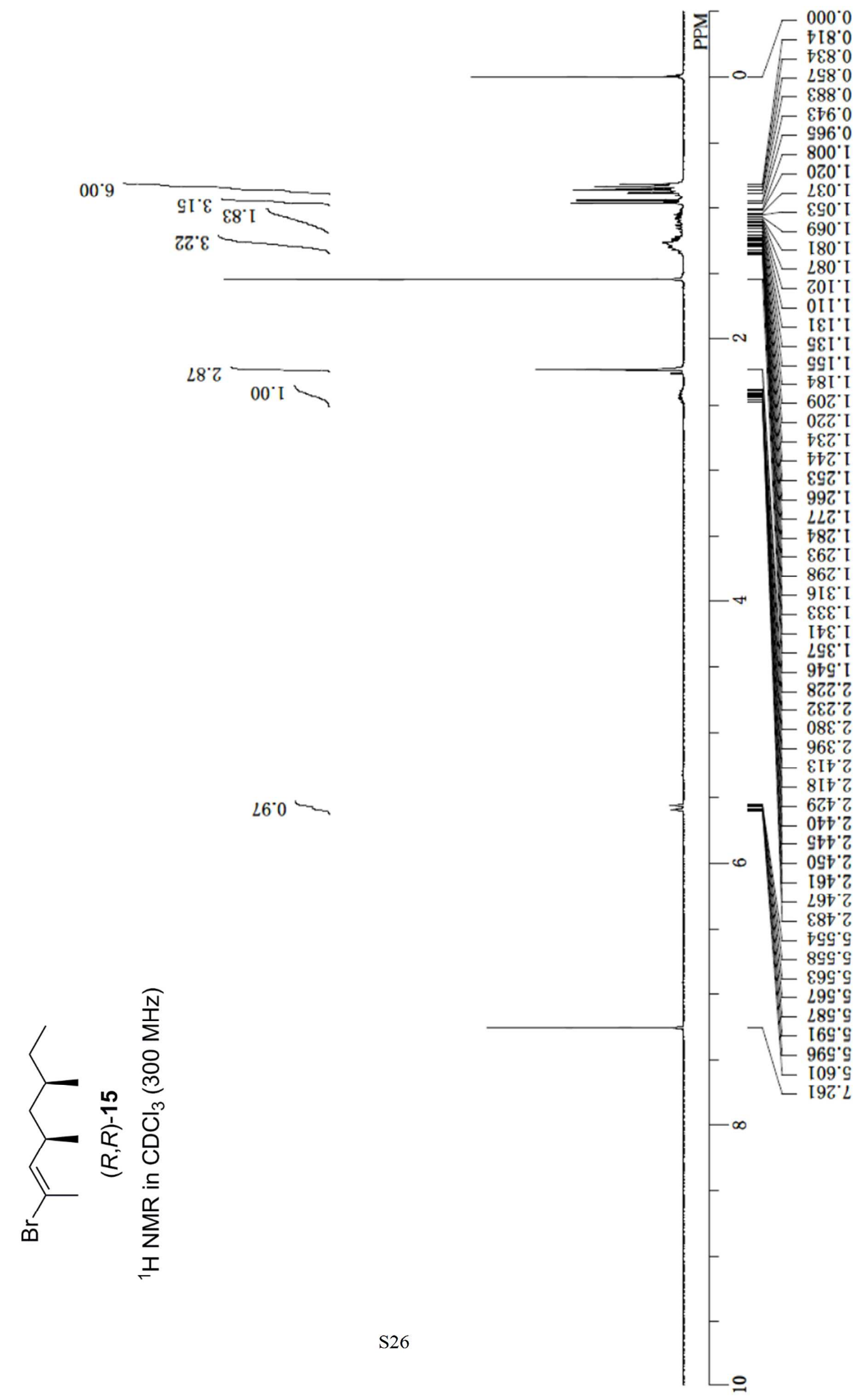




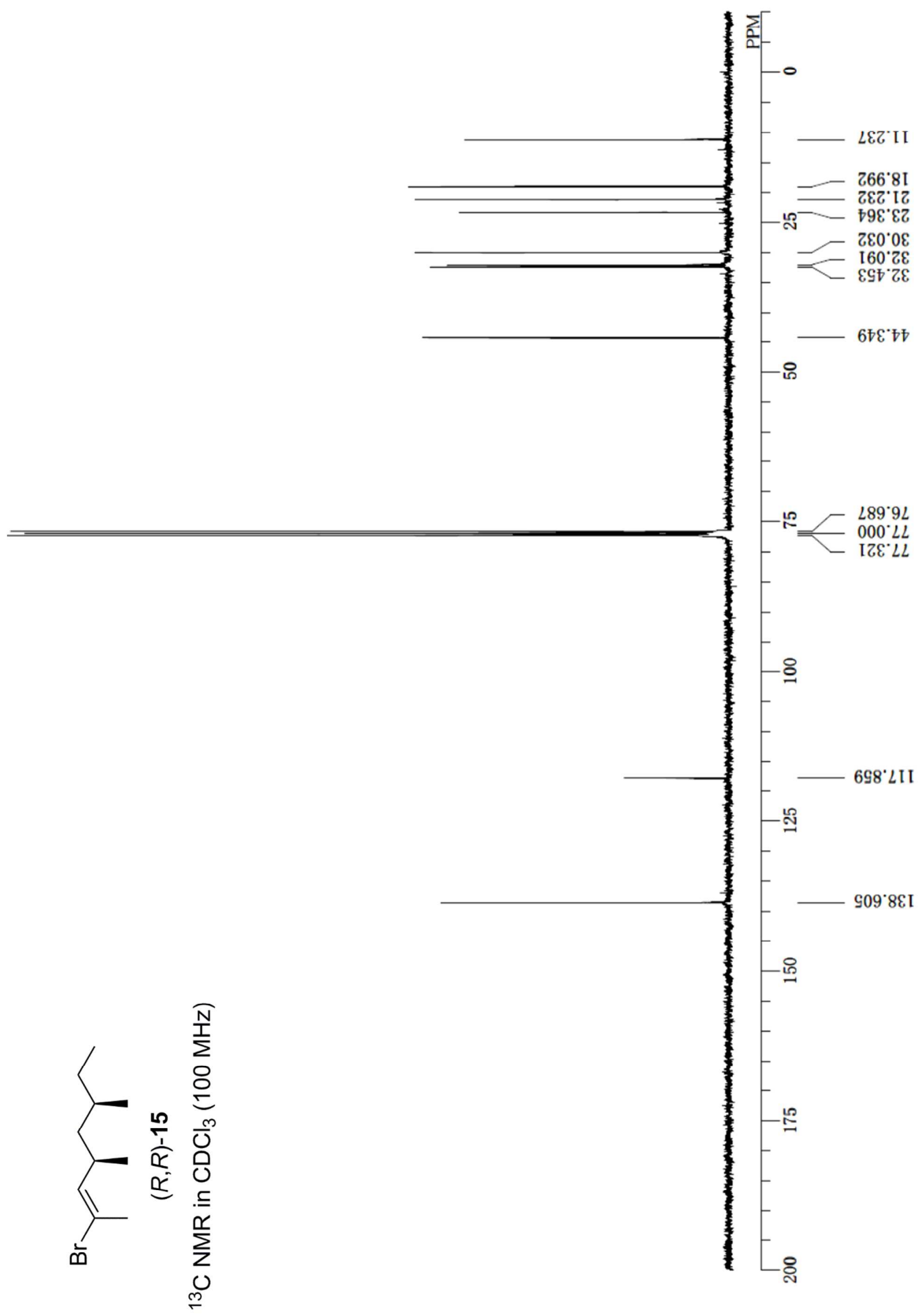




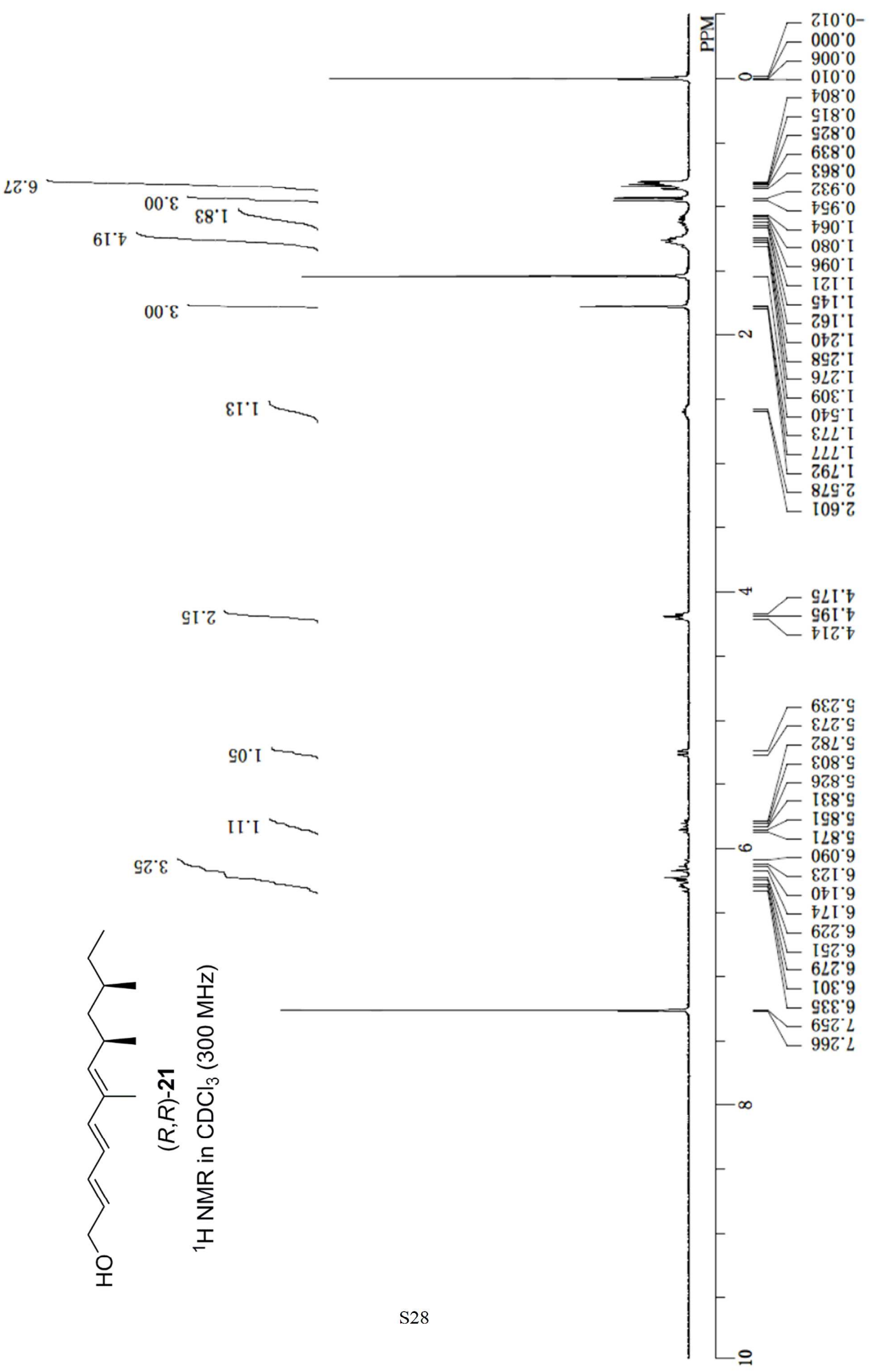




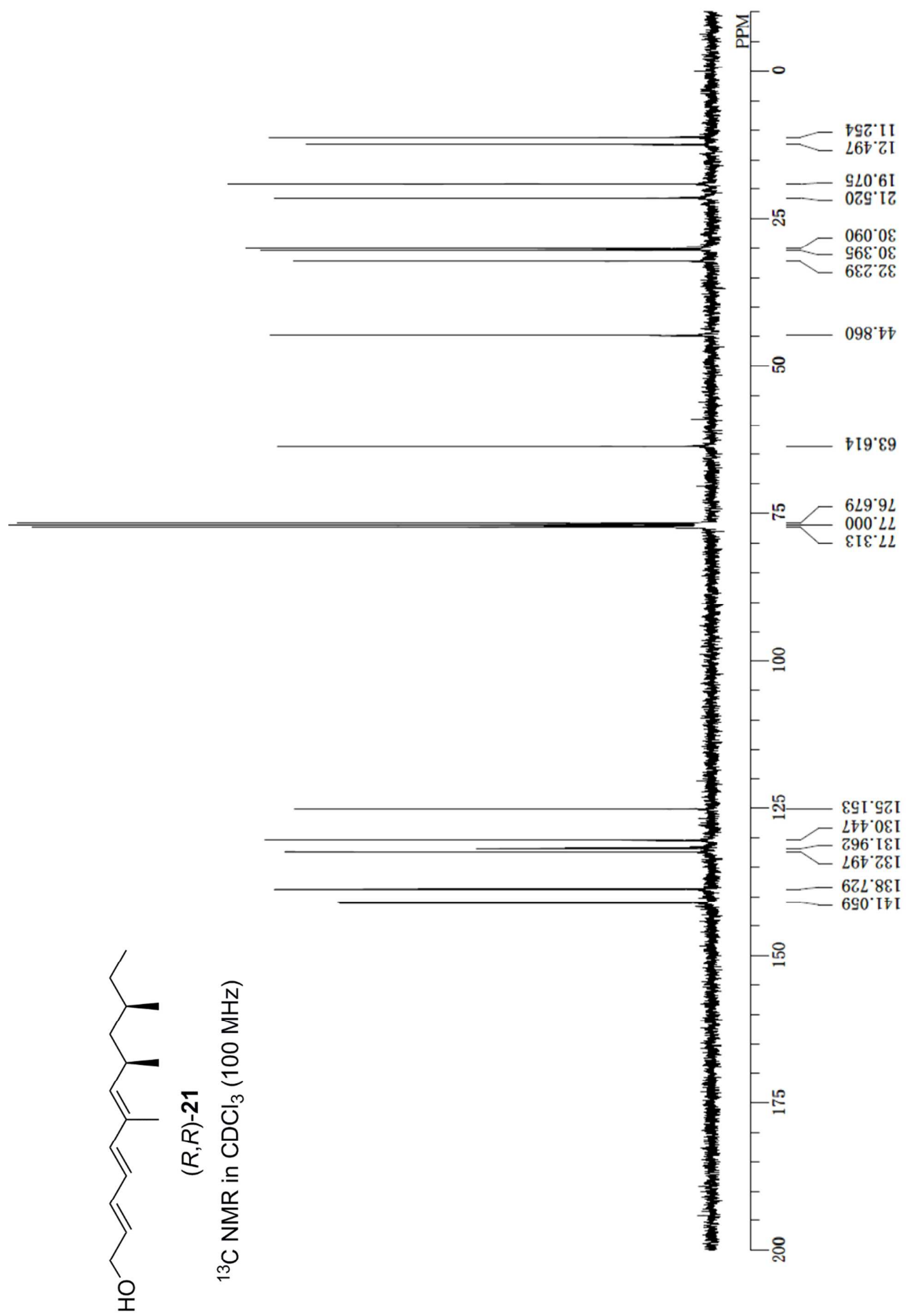



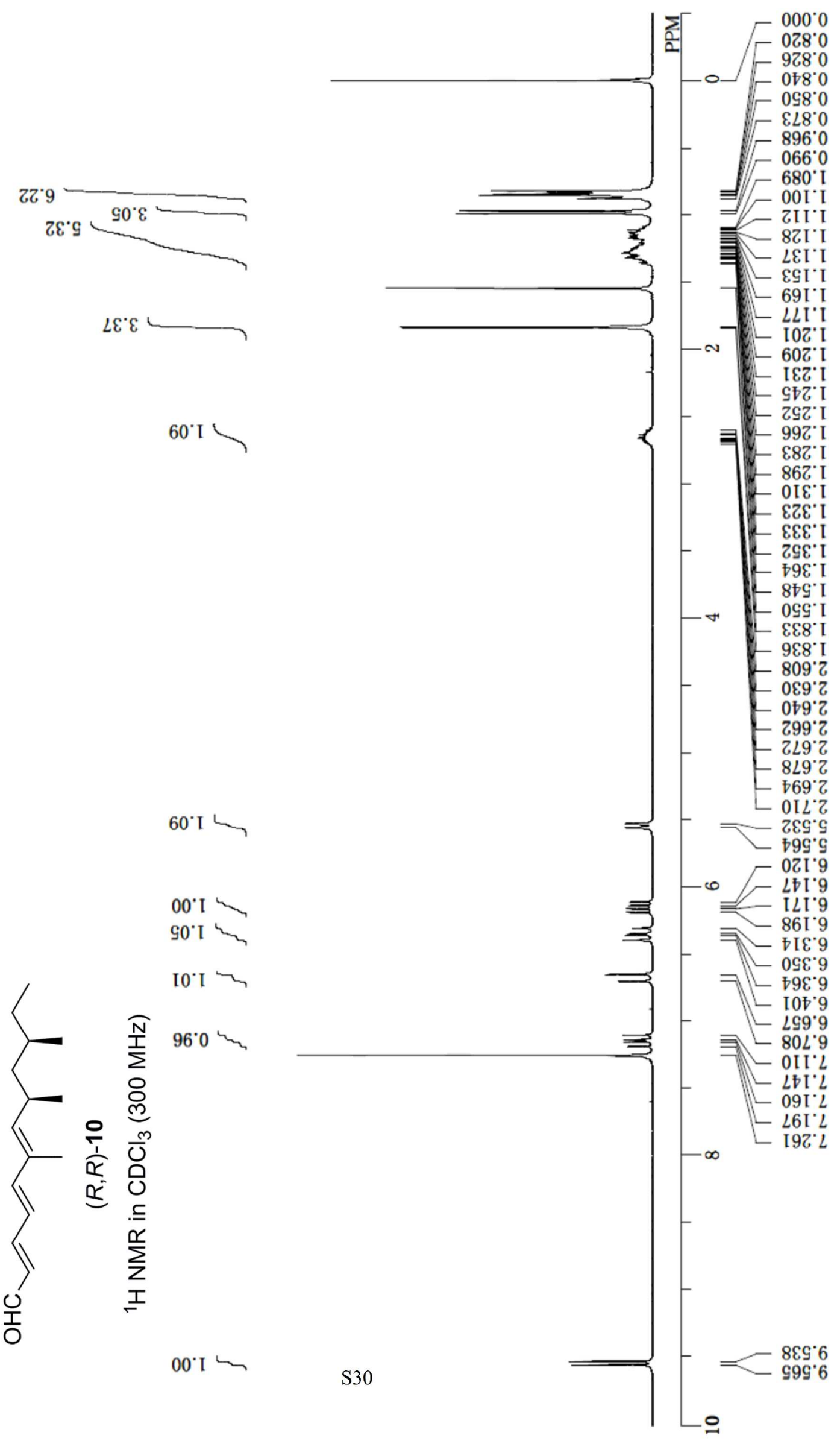


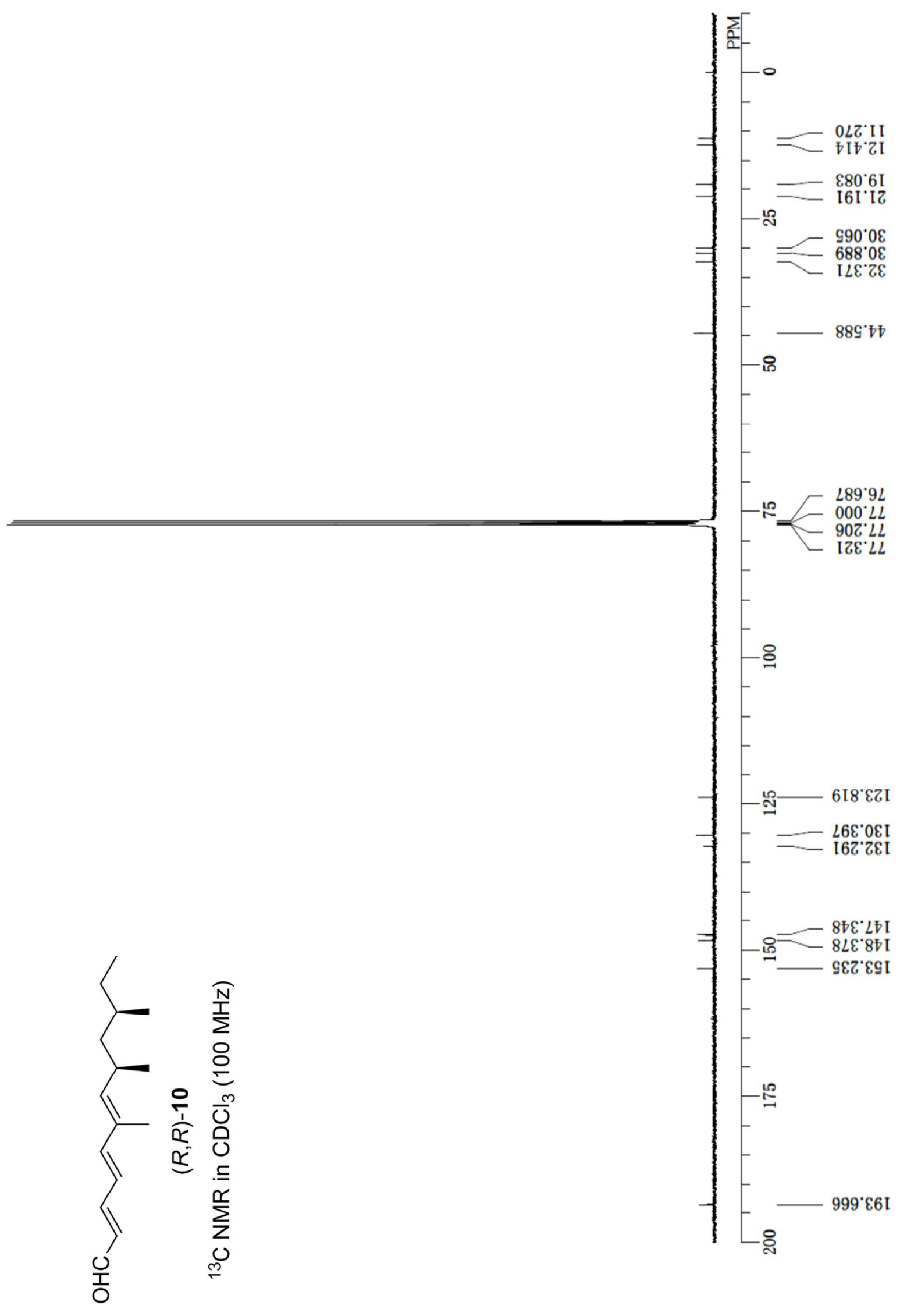



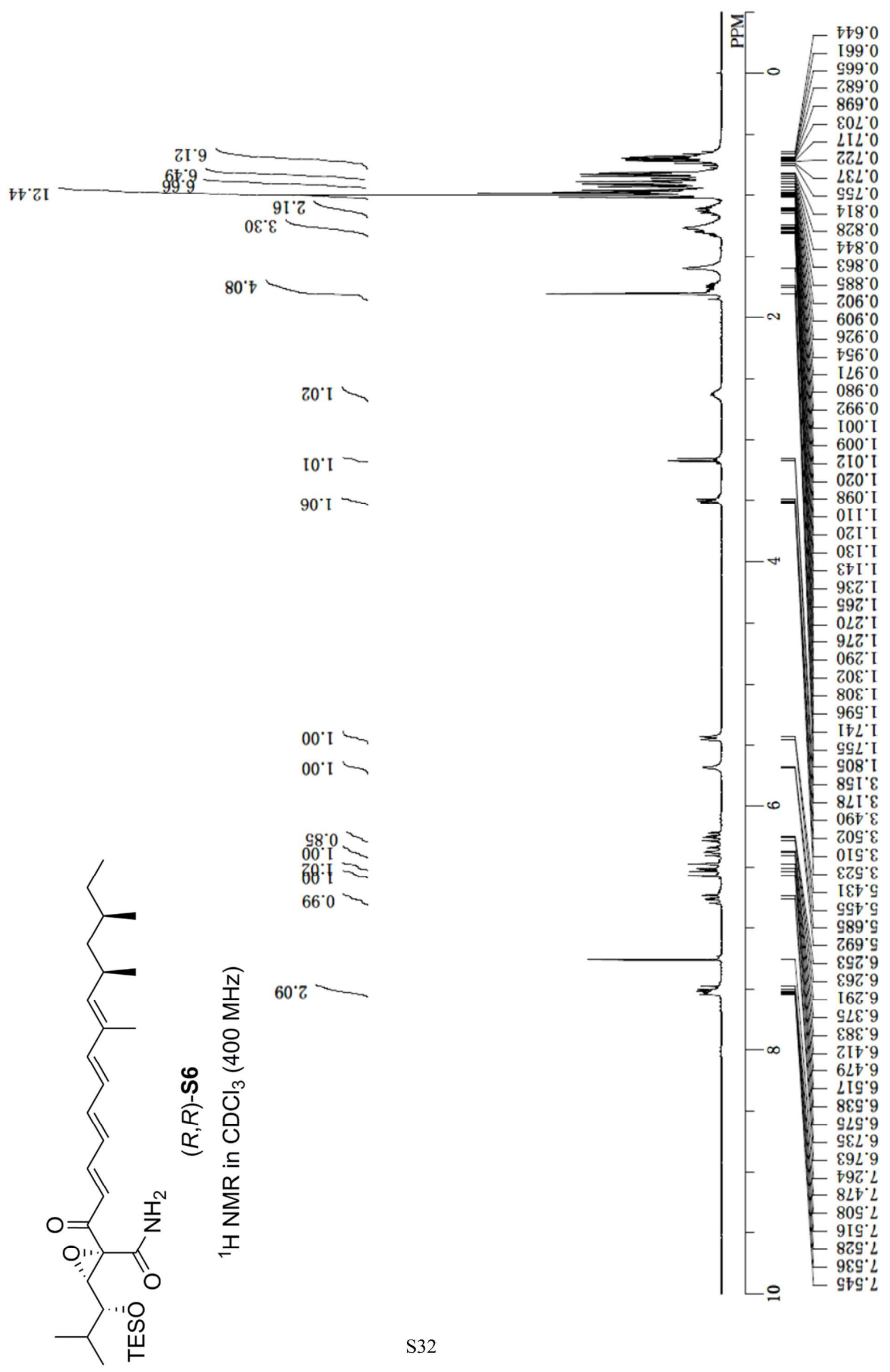


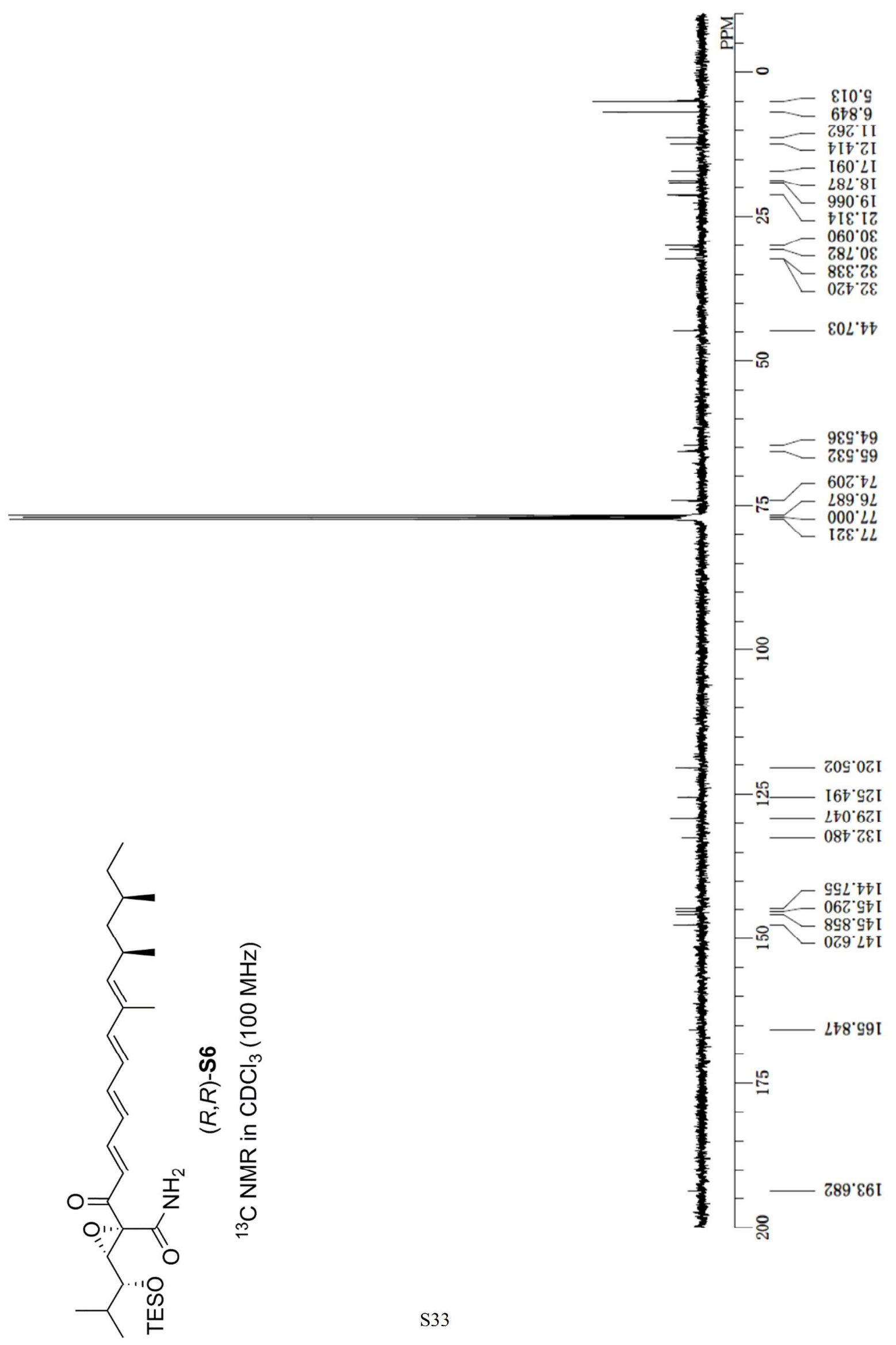




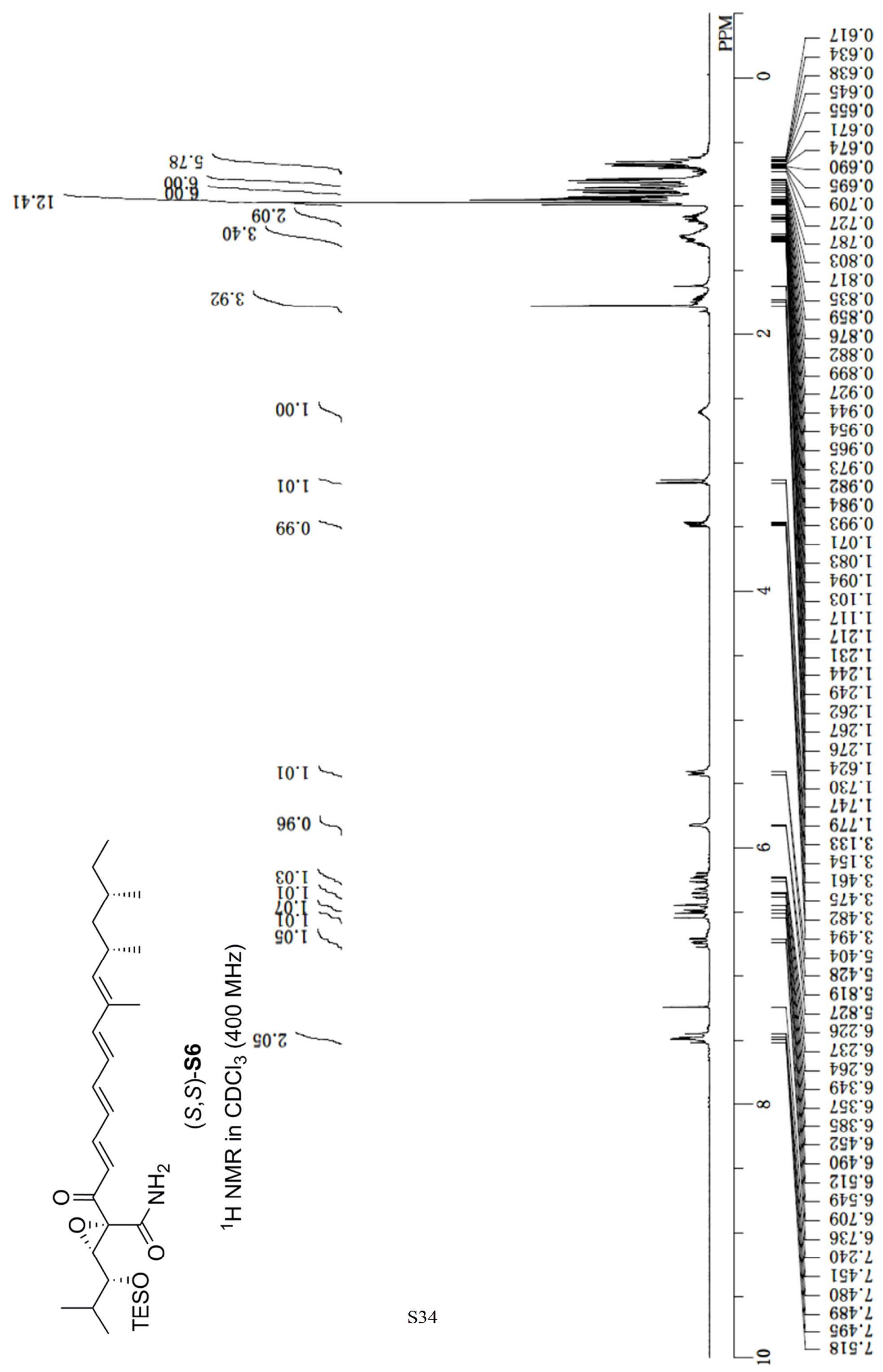




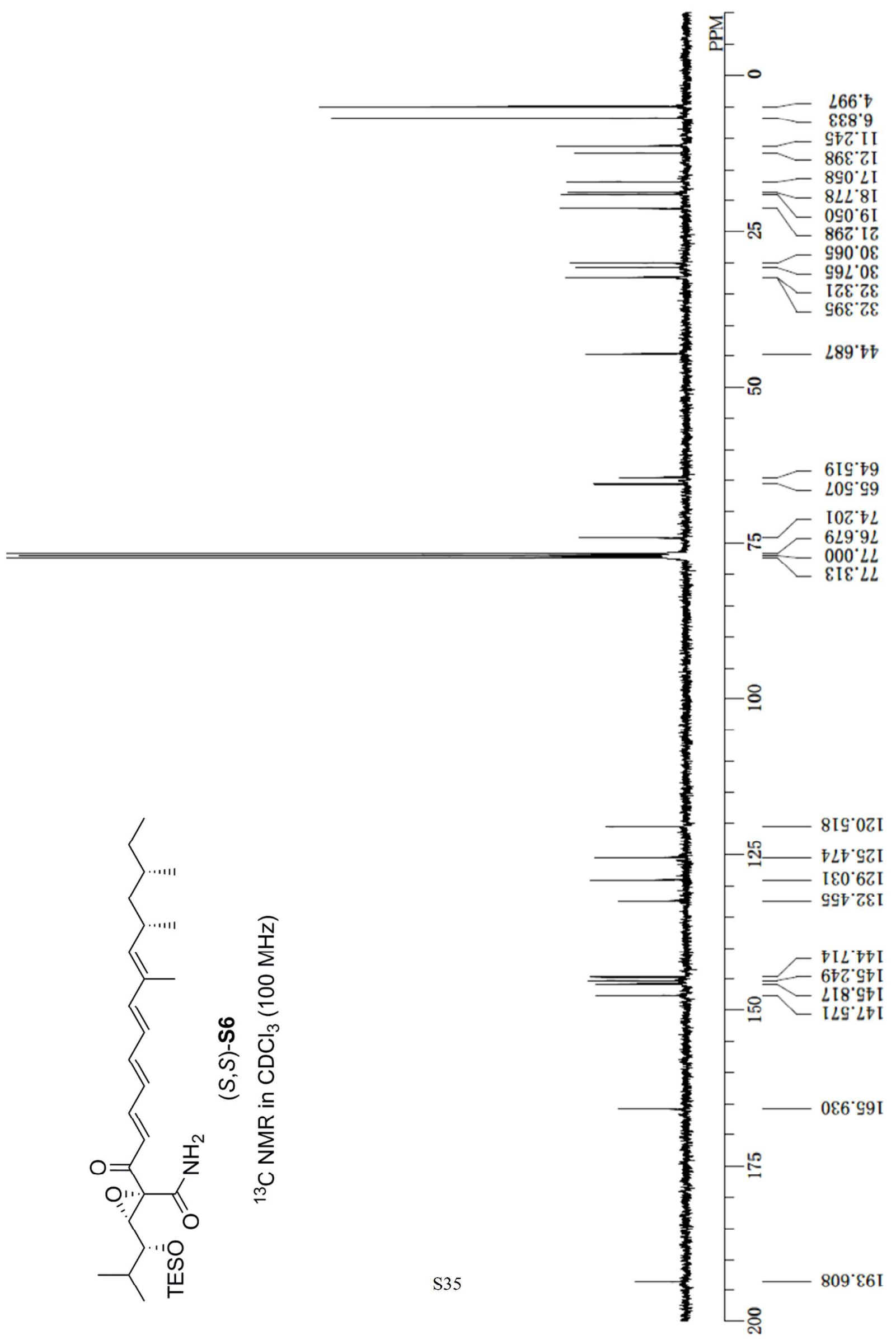




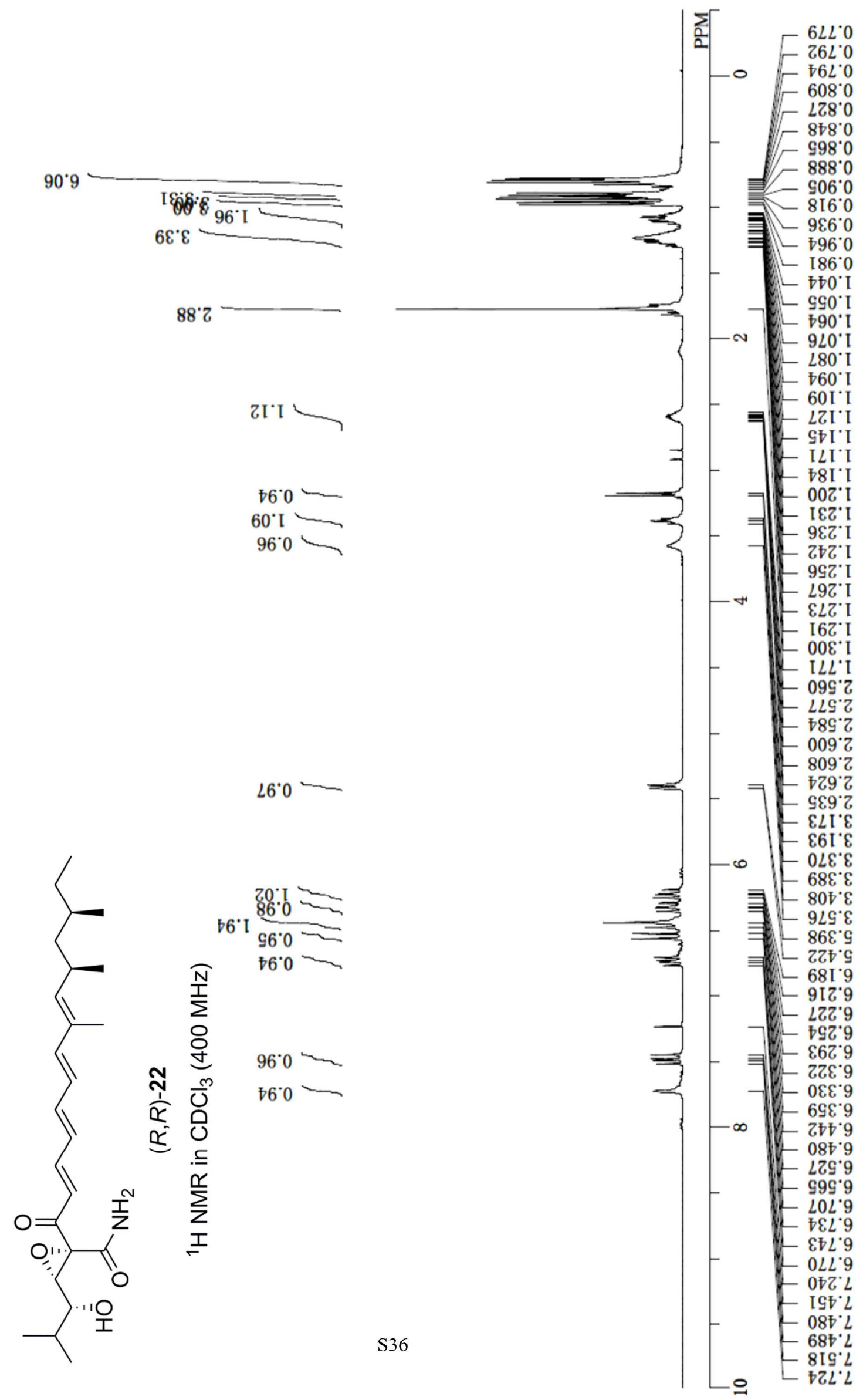




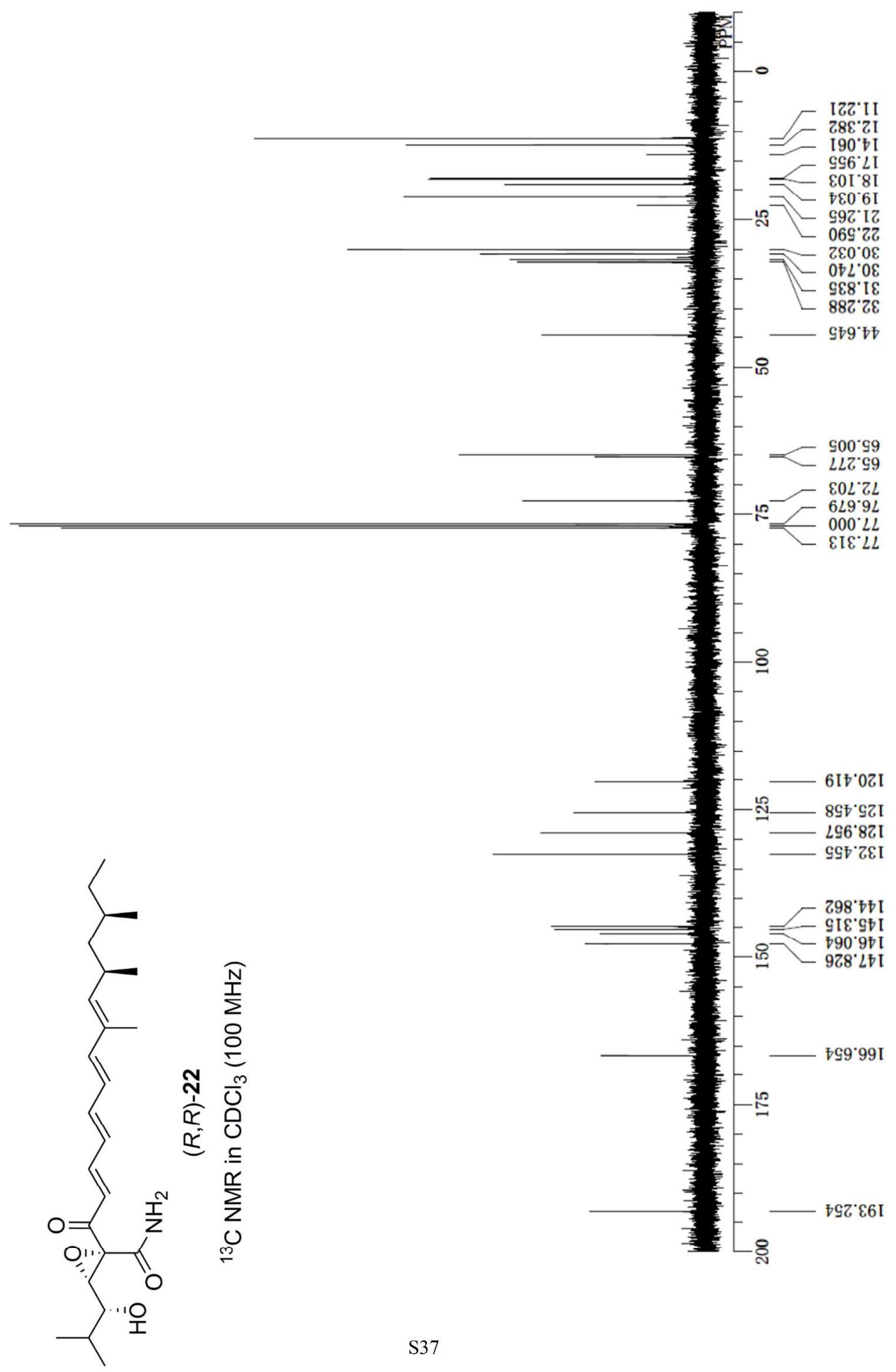


$90^{\circ} 9$

$86^{\circ} 2$

$\angle 6^{\circ} 0$

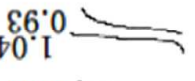

$260^{\circ}$

$30^{\circ} \mathrm{I}$

86.02

$\stackrel{\widehat{N}}{\Sigma}$

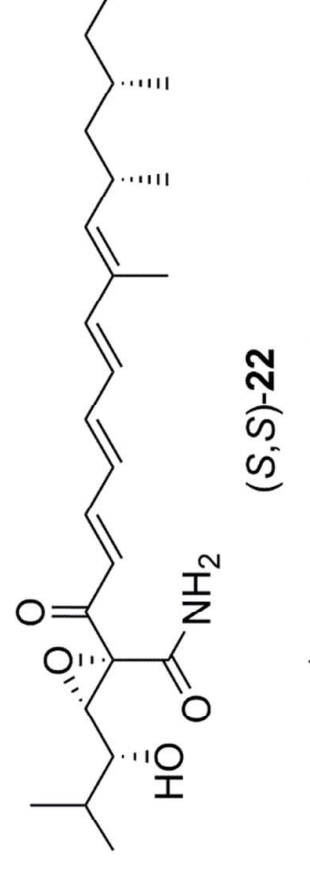

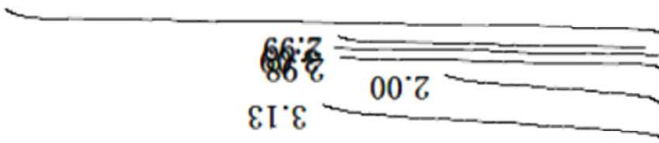

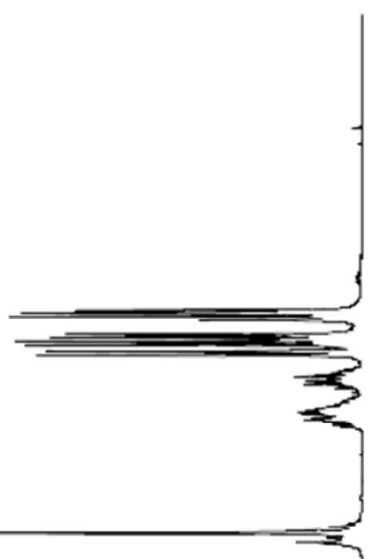

궁

$-\varepsilon 18^{\circ} 0$

- 0 F $878^{\circ} 0$

$\$ 76^{\circ} 0$

- It6.0

- $796^{\circ} 0$

F $026^{\circ}$

- 9I0. I

6to

$-060^{\circ}$

$\leftarrow 860^{\circ} \mathrm{I}$

$\leftarrow 0 \mathrm{III}^{\circ}$

- IZI'I

- OEI.

$-\varepsilon T_{1}$

- $39 \mathrm{I}^{\circ} \mathrm{I}$

- GIZ. I

- z\&Z. I

- ETR I

$-697^{\circ} \mathrm{I}$

- OLZ I

- 9L2.

(- 06z.

- $20 \varepsilon^{\circ} \mathrm{I}$

$-0 z \varepsilon^{\circ} \mathrm{I}$

$-97 \varepsilon^{\circ} \mathrm{I}$

- $9 \varepsilon \varepsilon^{\circ}$.

$208^{\circ} \mathrm{T}$
-

$-039^{\circ}$

- $289^{\circ} \mathrm{Z}$

- 万t9.

$-669^{\circ} 2$

$-8 \mathrm{gI}^{\circ} \mathrm{\varepsilon}$

$-76 \mathrm{I}^{\circ} \mathrm{\varepsilon}$

$-\succsim \mathrm{I} \cdot \mathcal{E}$

$-\varepsilon\left[\sigma^{-} \varepsilon\right.$

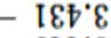

$-6 \hbar \hbar^{\circ} \varepsilon$

ᄂ $8 \varepsilon^{\circ} \mathrm{g}$

$-897^{\circ} \mathrm{c}$ $66^{\circ} 0$

$66.8=$

$66.0 \div$

$-97 I^{\circ} 9$

$-29 \Gamma^{\circ} 9$

- $677^{\circ} 9$

$-997.9$

$-762.9$

- $\succsim \varepsilon \varepsilon^{\prime} 9$

- $89 \varepsilon^{\circ} 9$

$-0 L \varepsilon^{\circ} 9$

$-00 t^{\circ} 9$

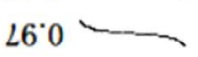

$260^{\circ}$

$-20^{\circ} \cdot$

- $879^{\circ} 9$

- $260^{\circ}$

- 092.9

$-8 L L .9$

$98 L .9$

- 18.9

$-897^{\circ} 2$

$-266^{\circ} \mathrm{L}$

$-989^{\circ} 2$

$-\pi 89^{\circ} 2$

$-799^{\circ} 2$

- ESL.2

-092.2
$-\quad 120.8$ $\left\llcorner 297^{\circ} 9\right.$ 


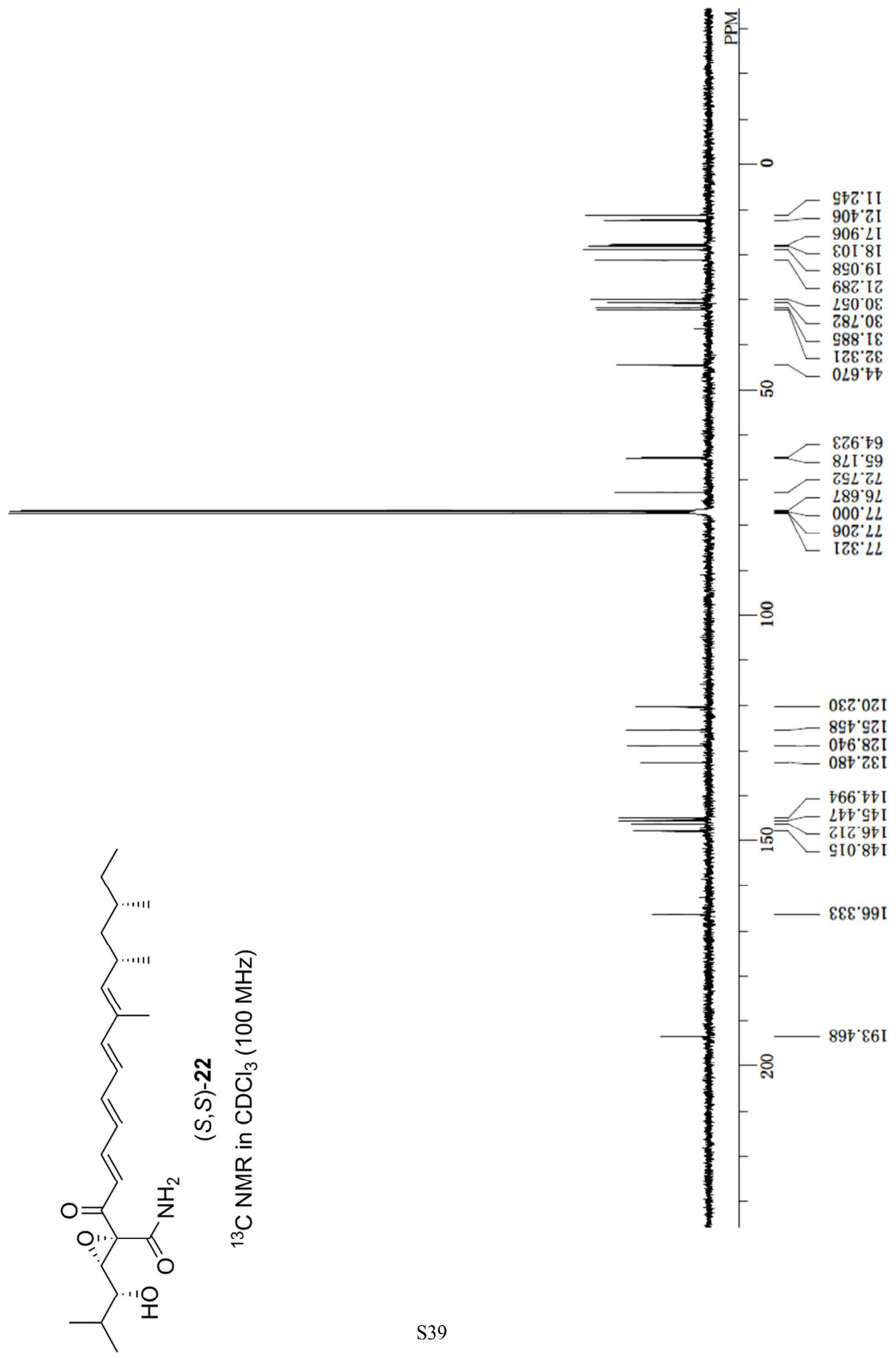




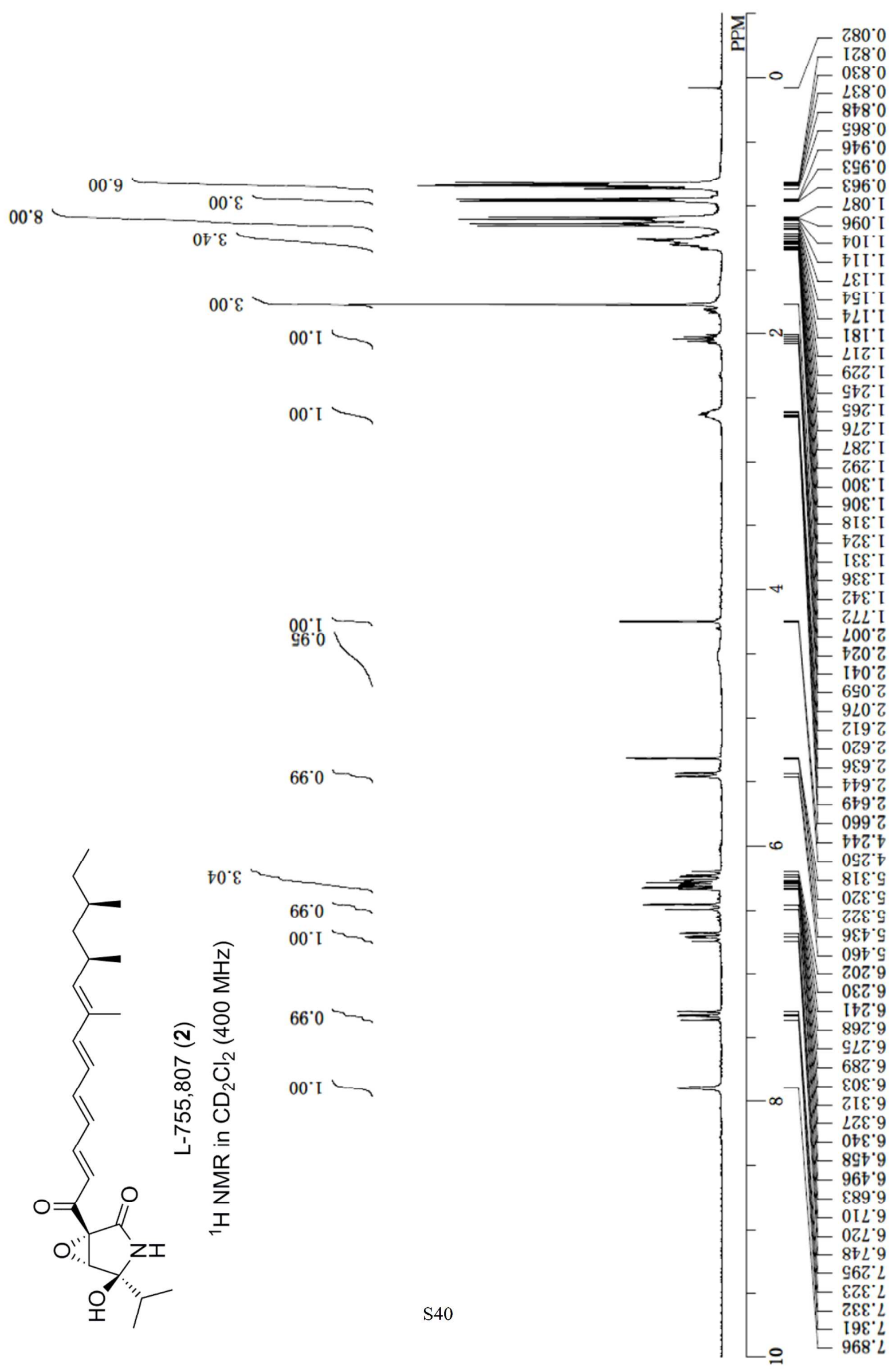




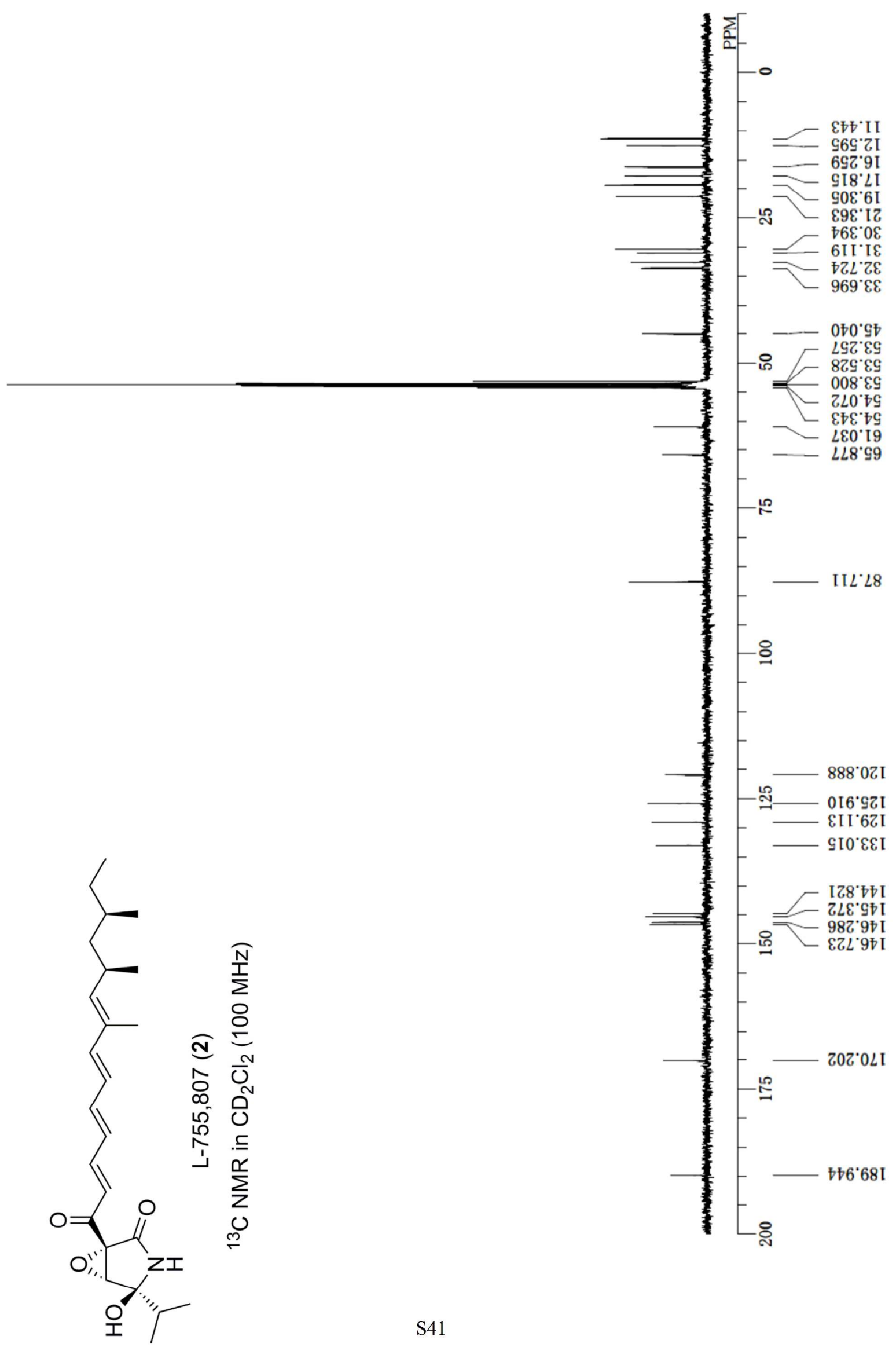




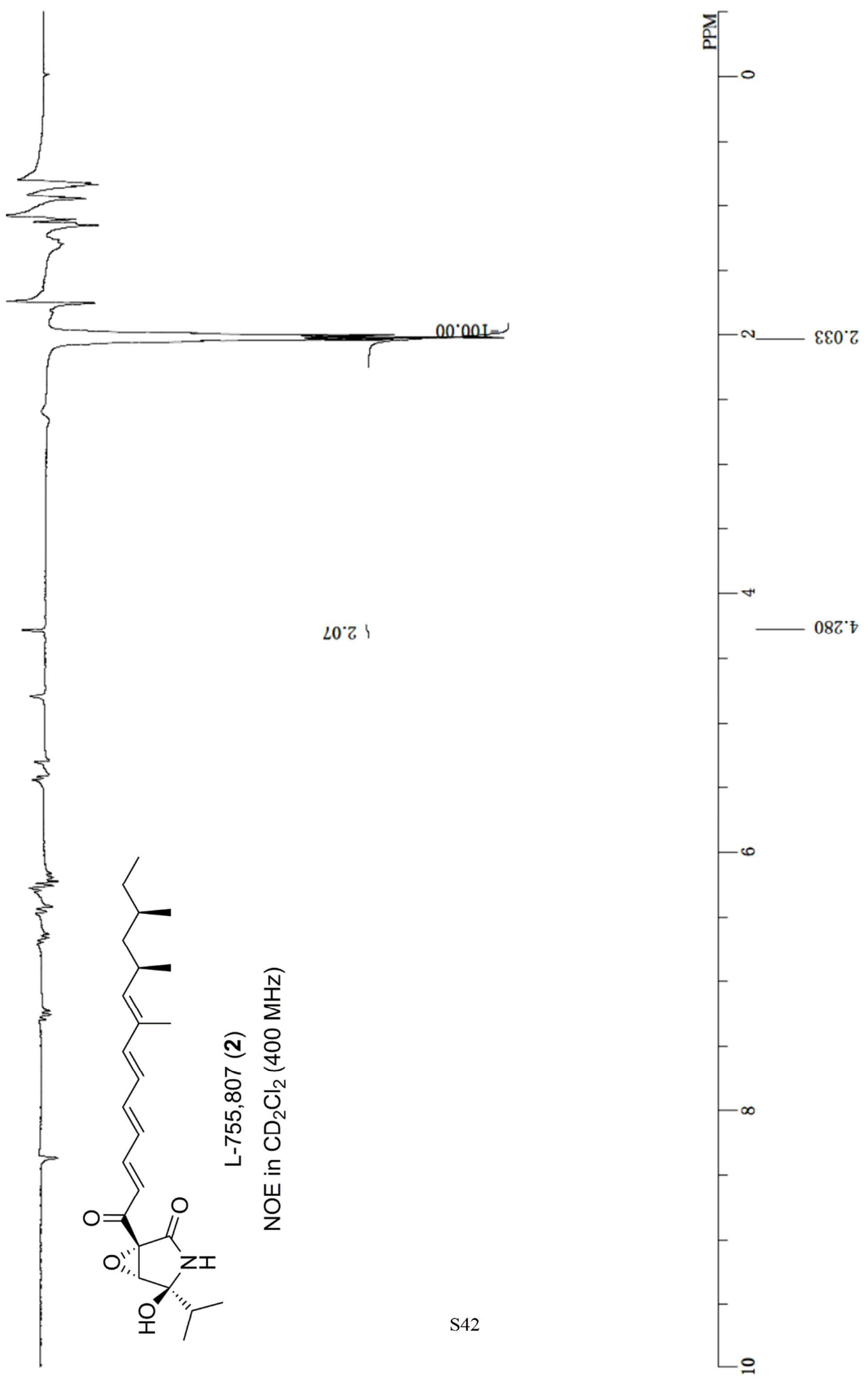




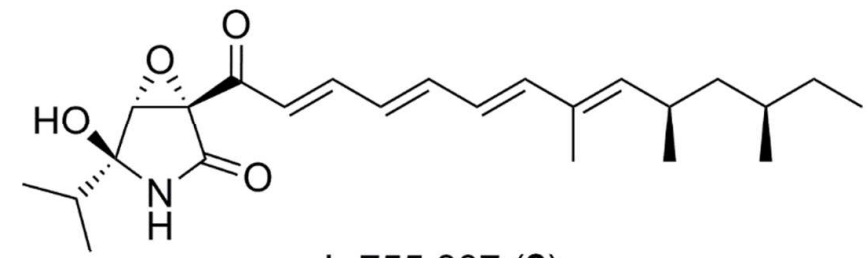

L-755,807 (2)

NOESY in $\mathrm{CD}_{2} \mathrm{Cl}_{2}(500 \mathrm{MHz})$

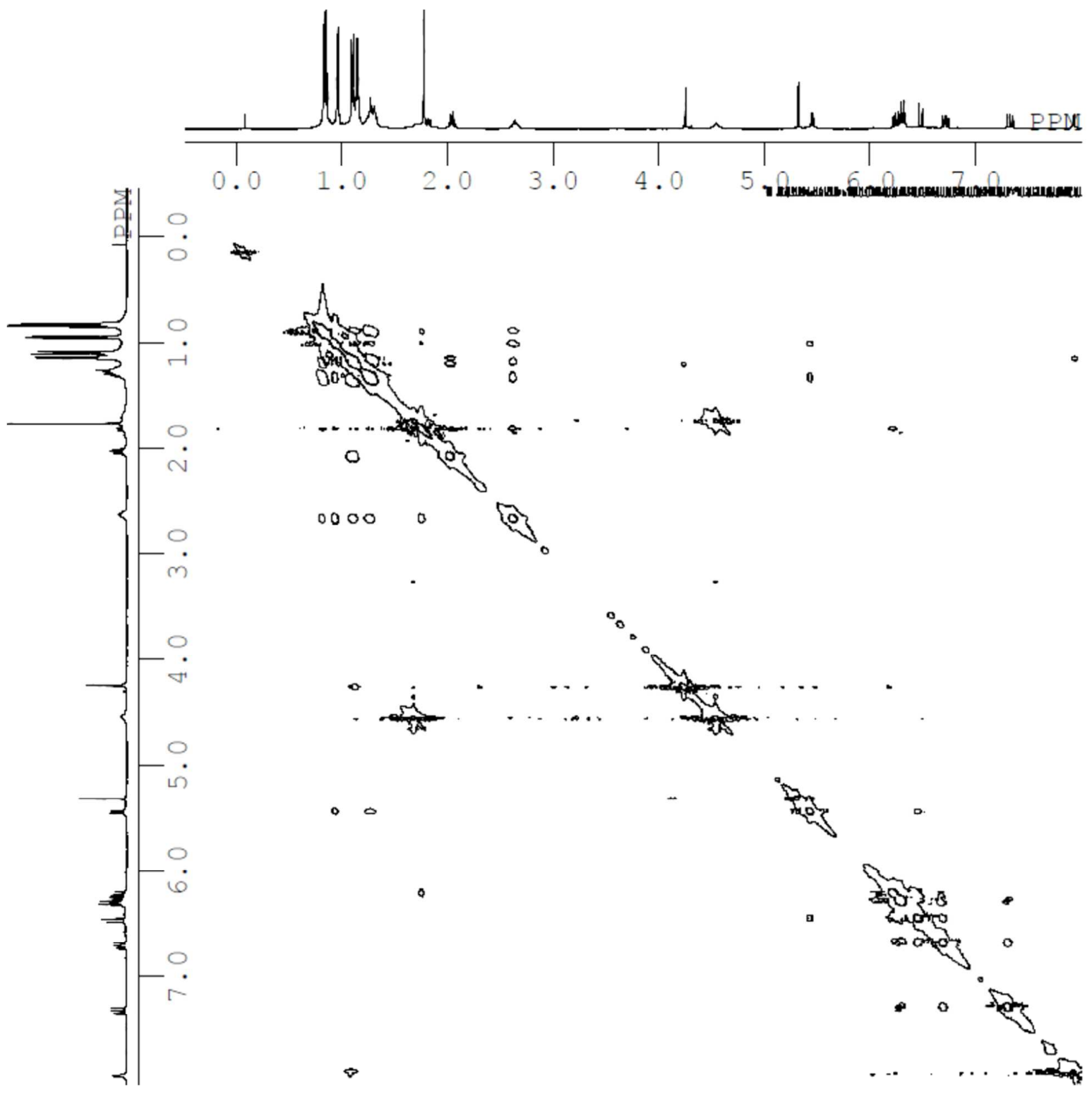




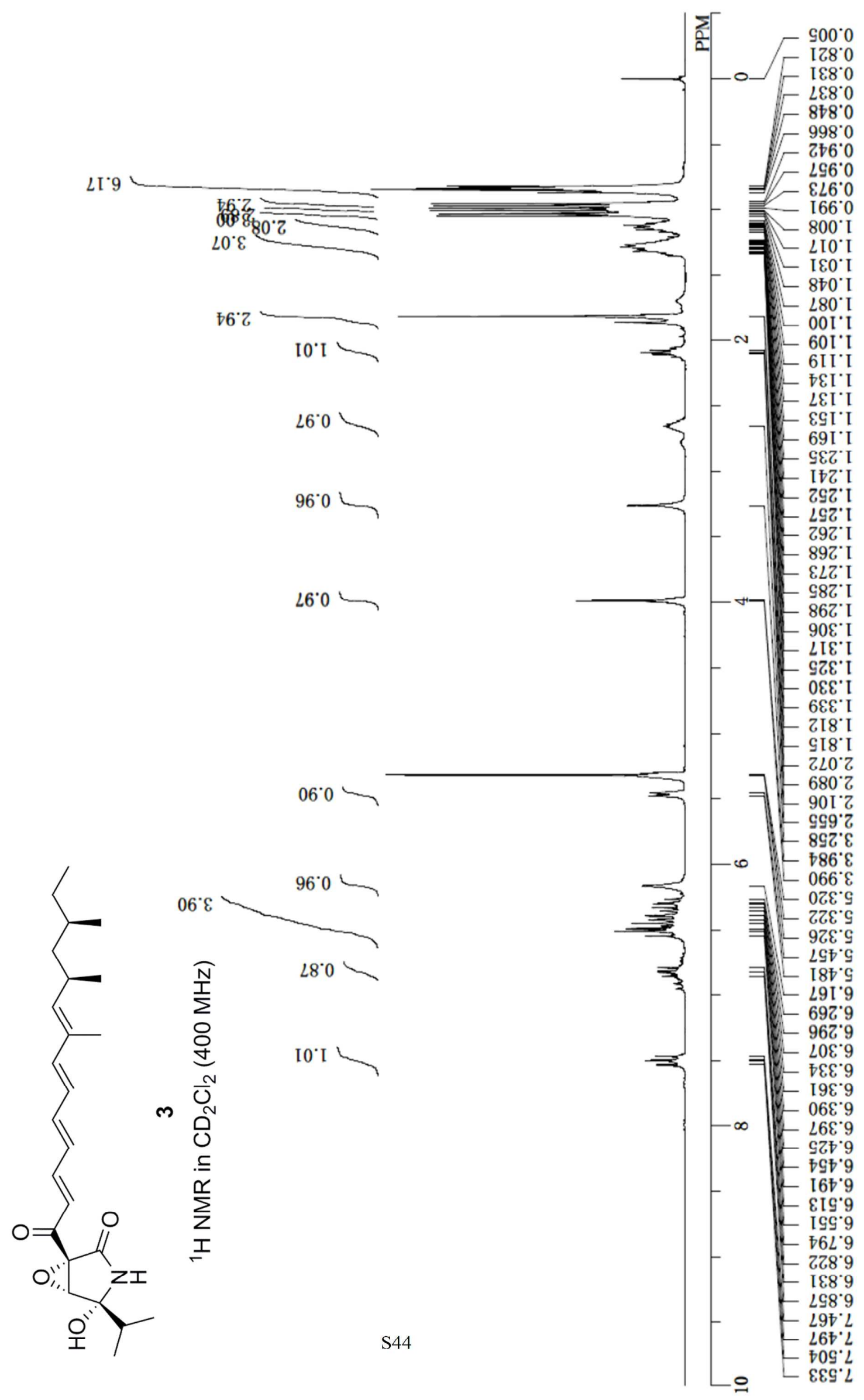




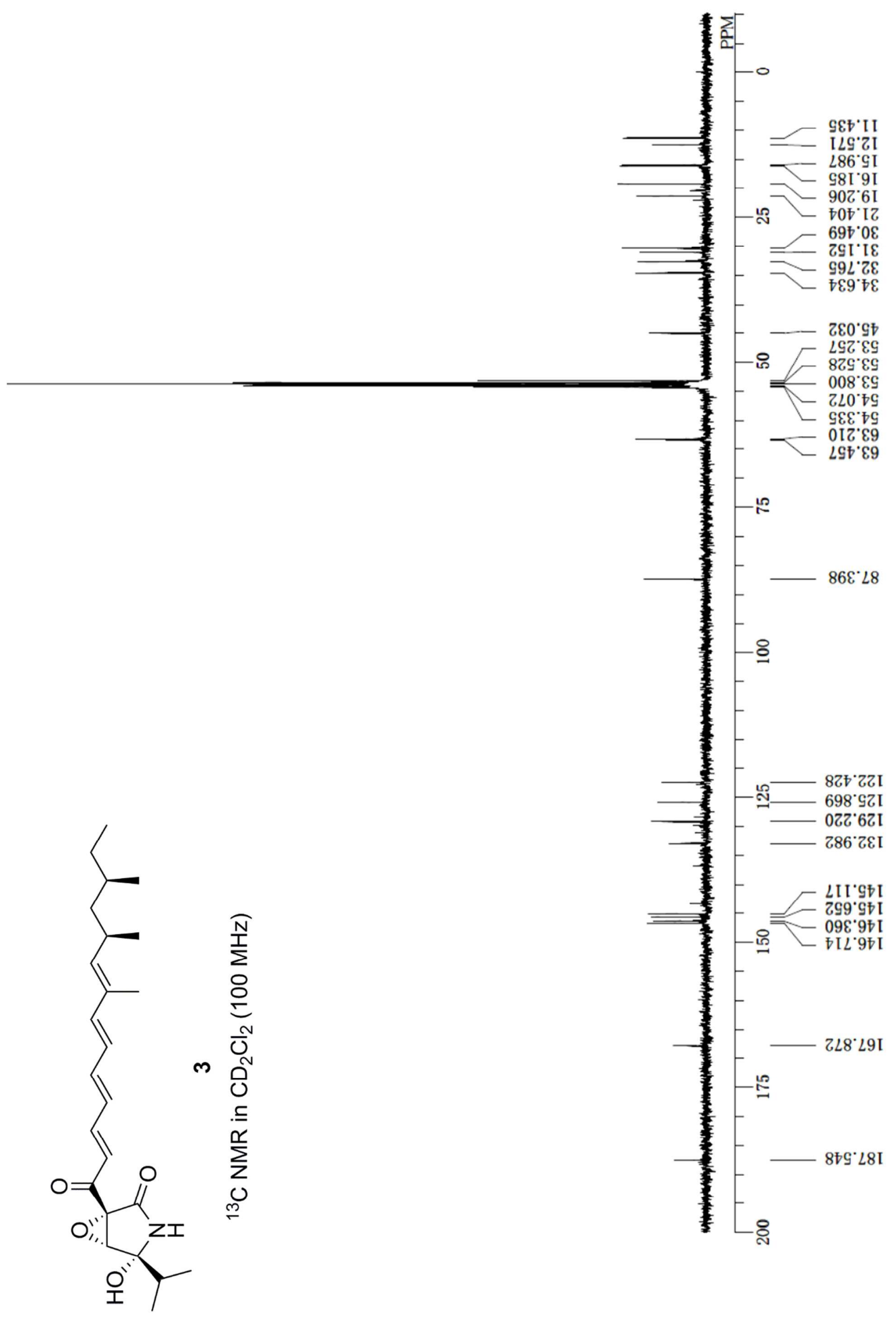




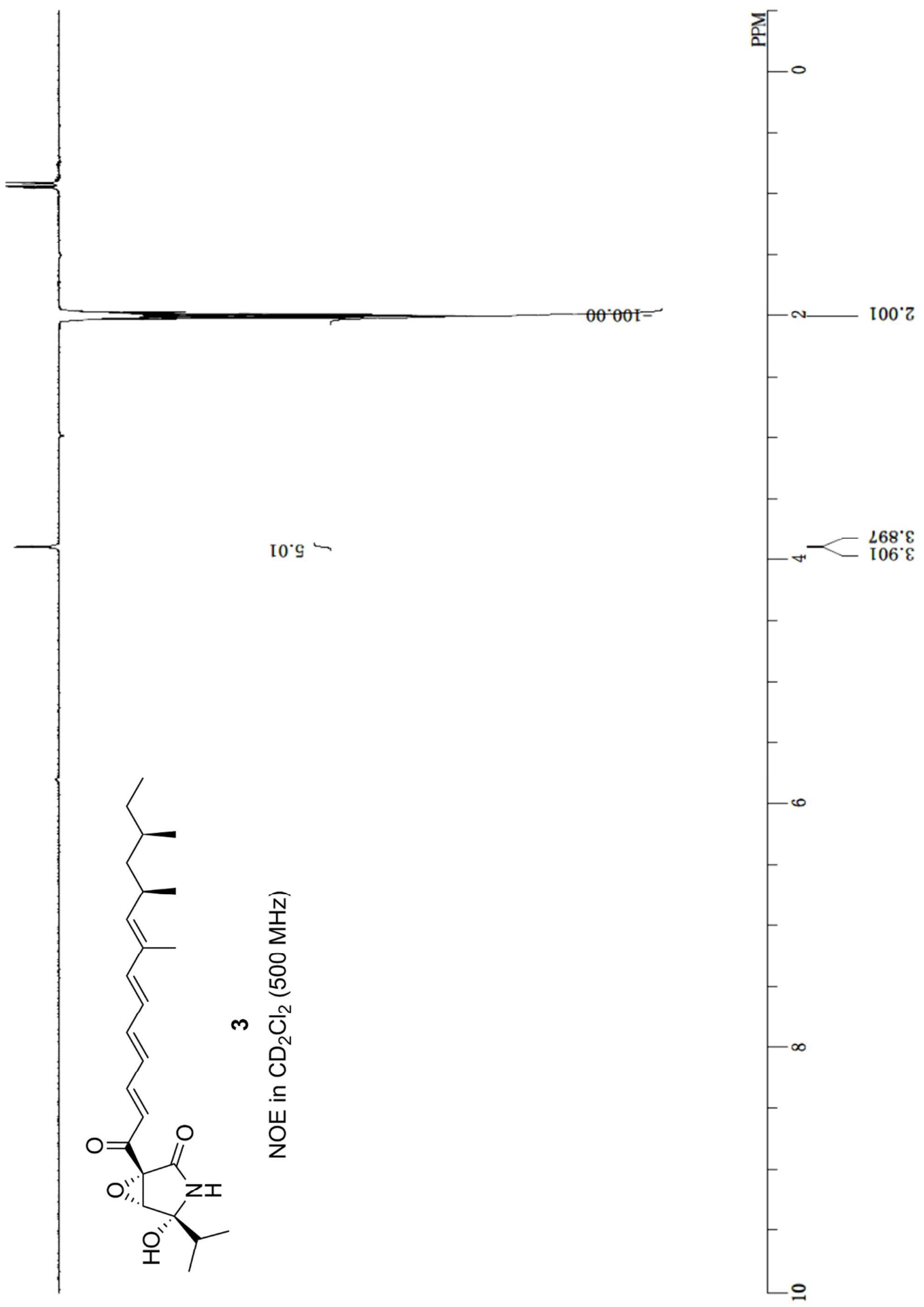




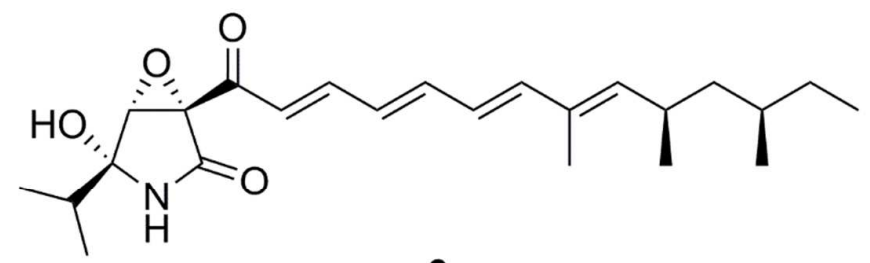

3

NOESY in $\mathrm{CD}_{2} \mathrm{Cl}_{2}(500 \mathrm{MHz})$

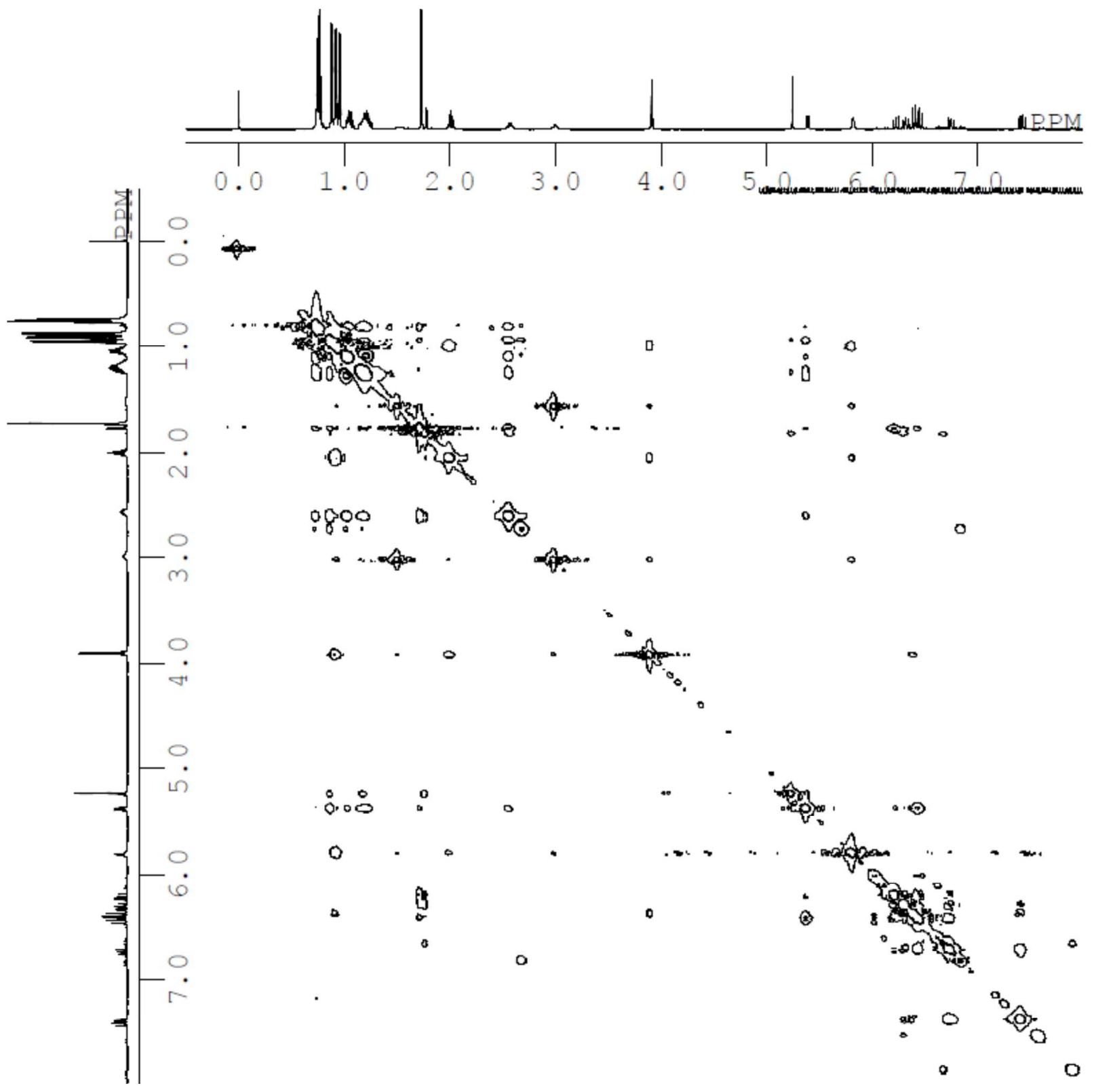



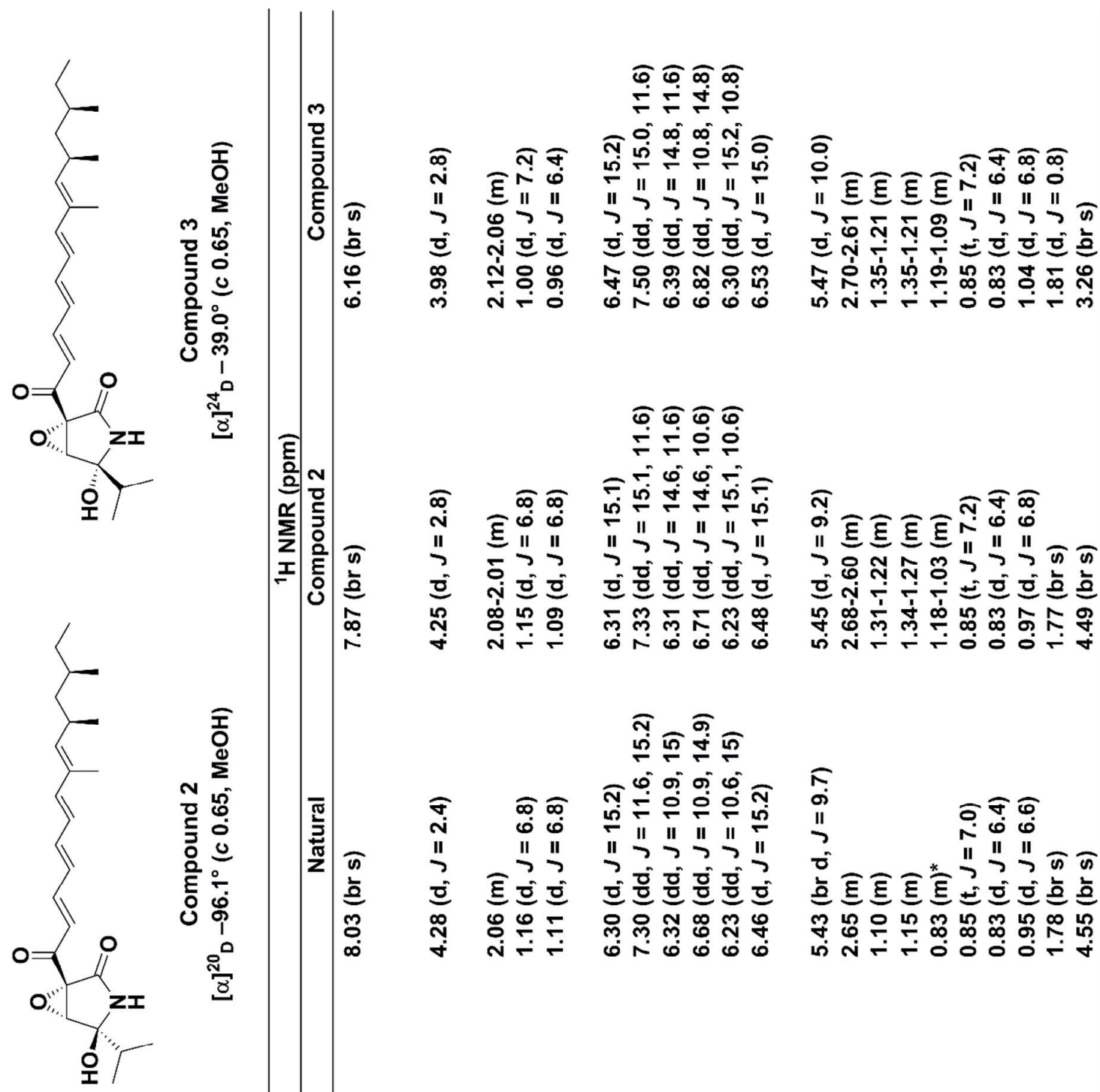

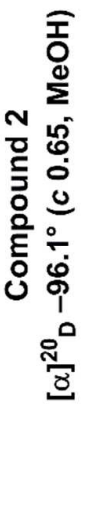

बढ़

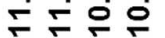

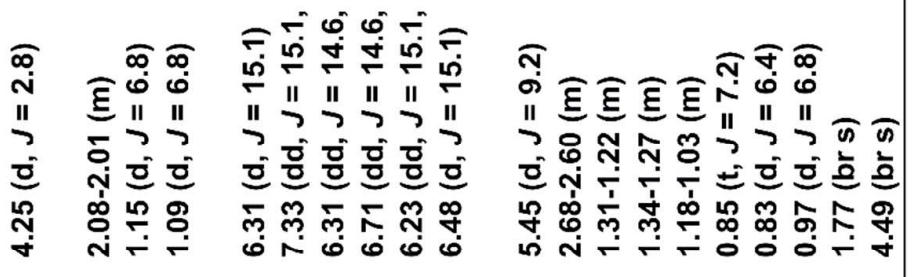

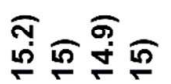

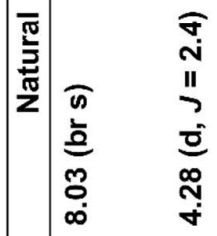
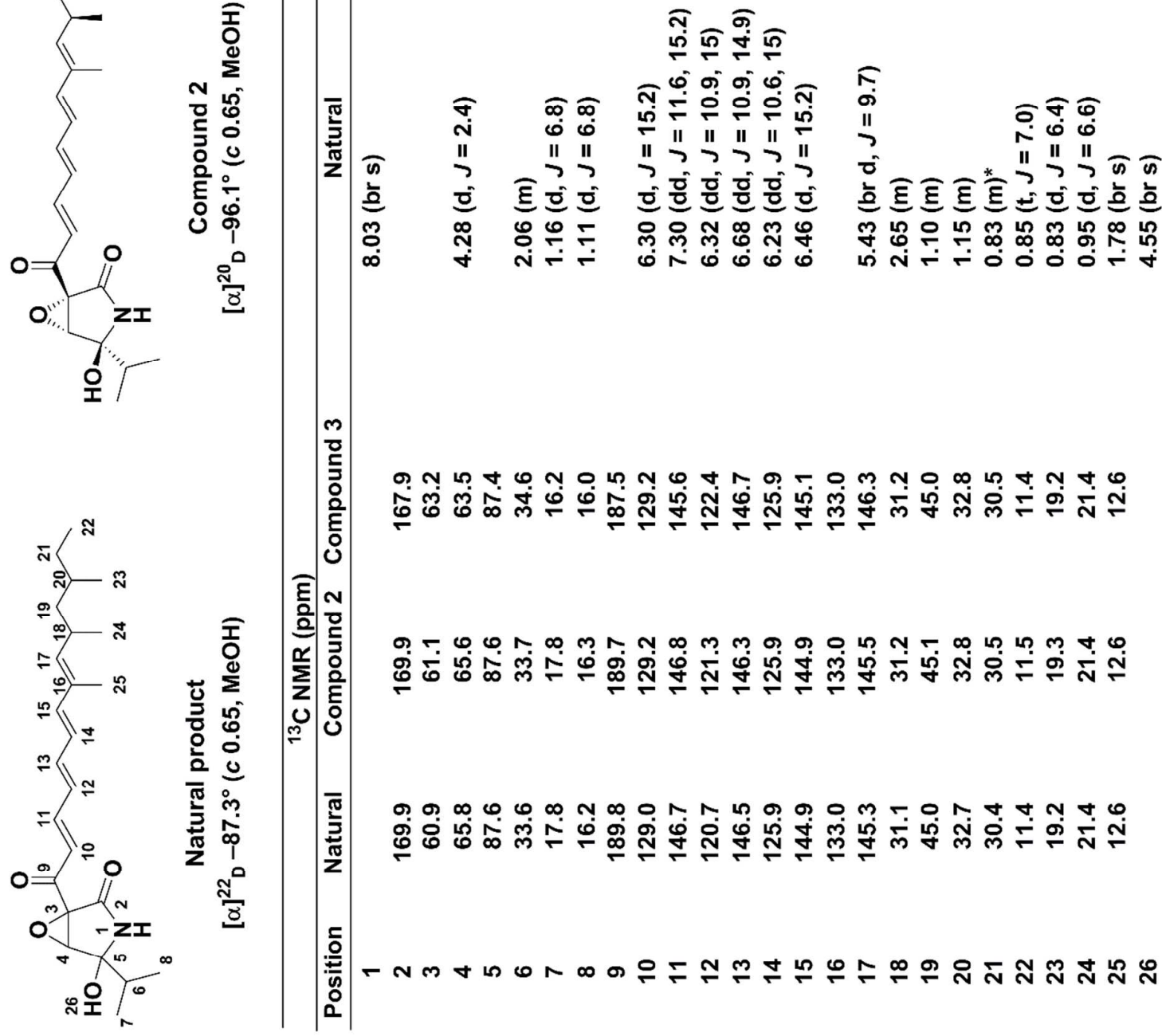

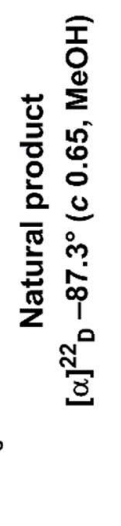

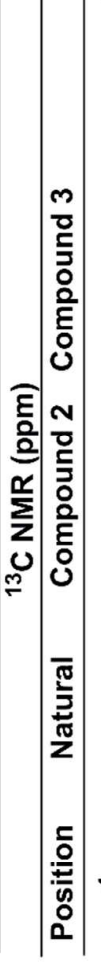

อ 4

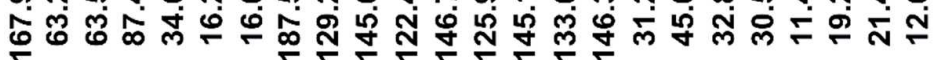

ด 유

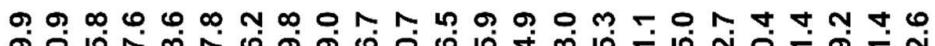
ஜㅇㅇ - Nm+แ 


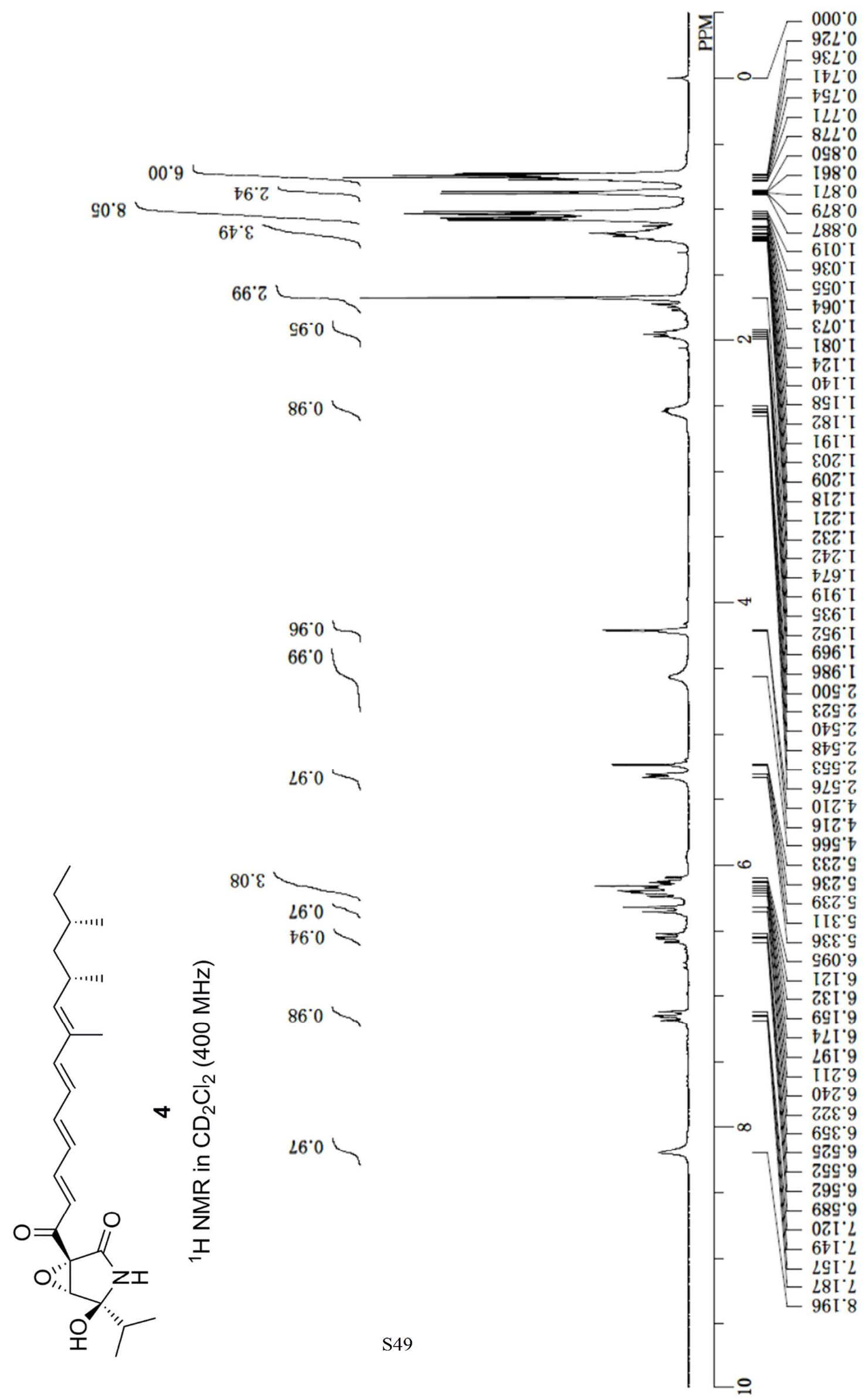




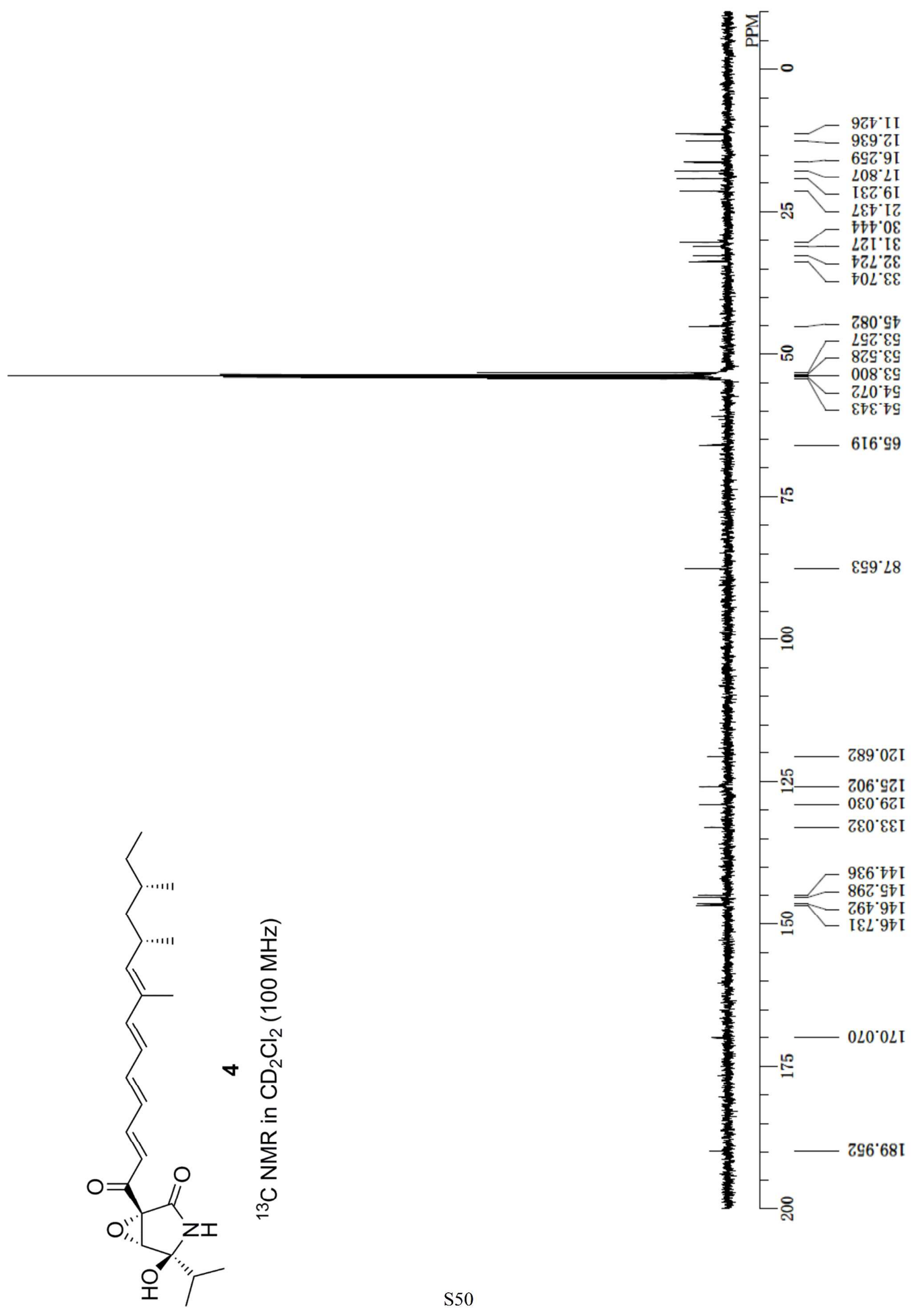




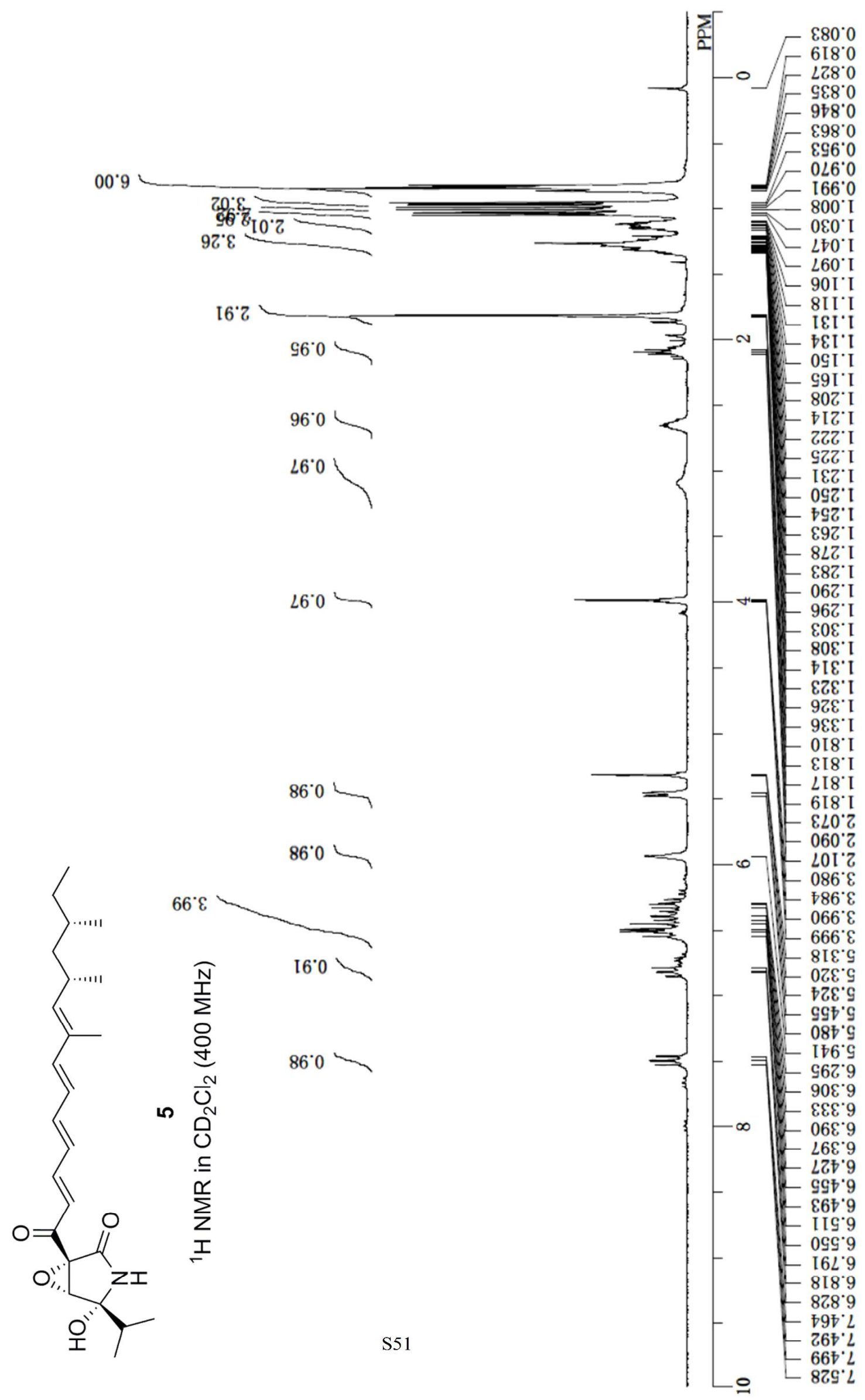




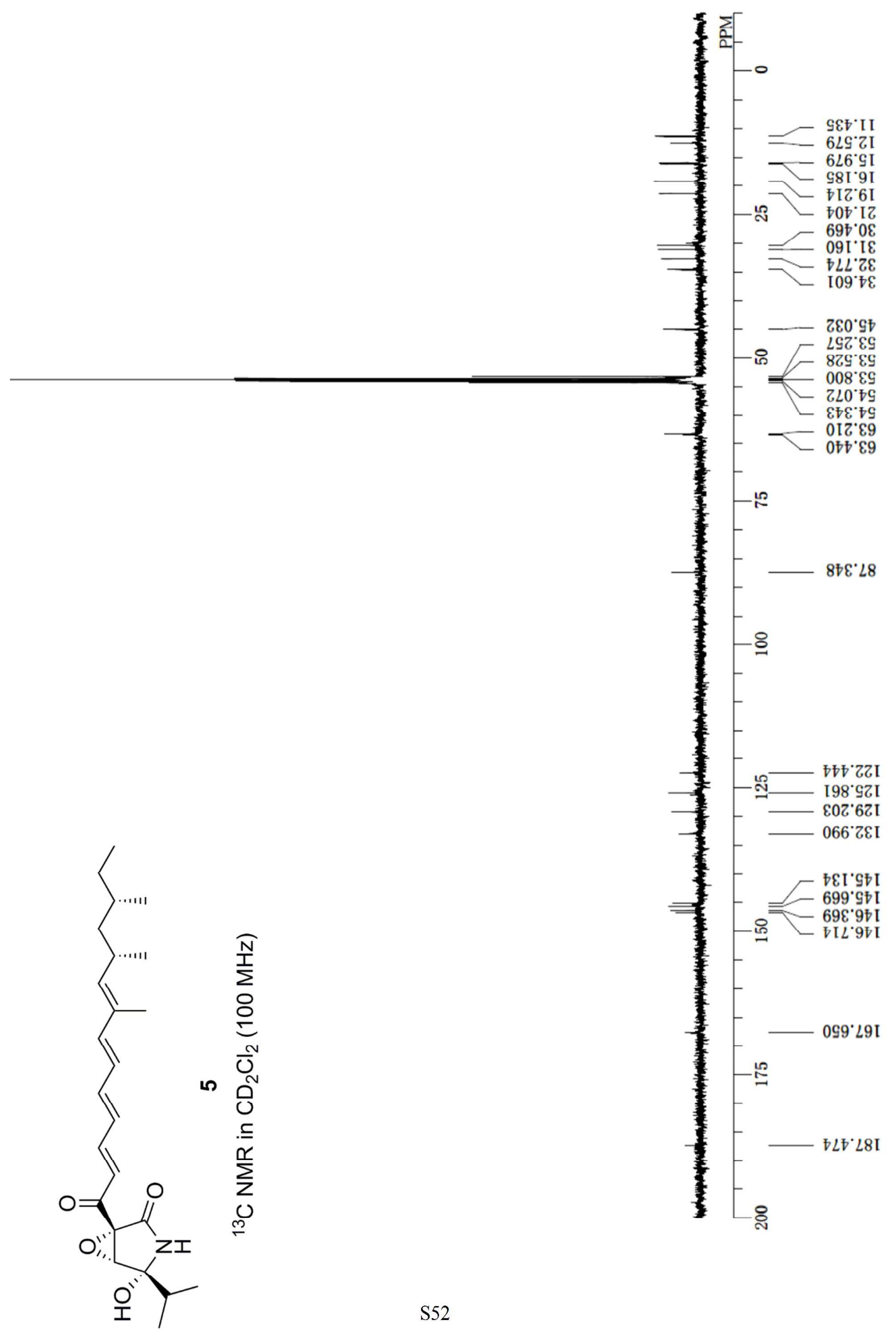



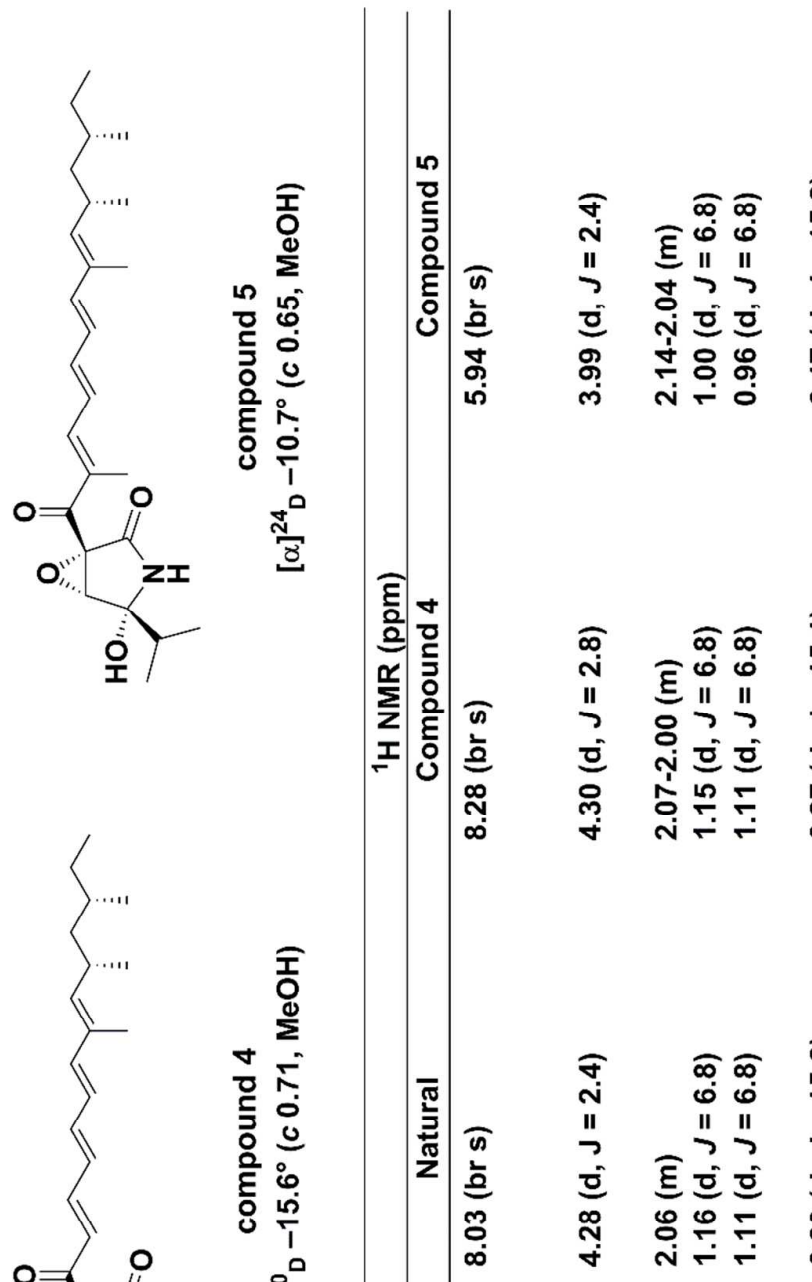

क)

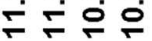

งิ่

แก

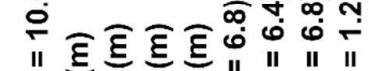

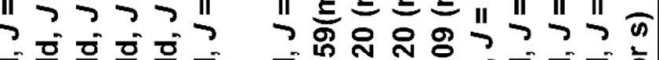

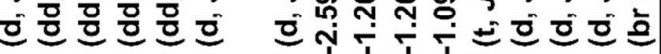

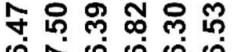

守穴

\section{ชิ่า}

$F F F$

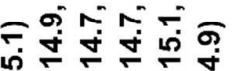

की - II

"ᄀ ว ว ᄀ" II

후흥 훙 훙 흥후

¿ำ

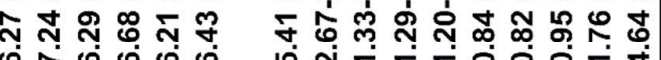

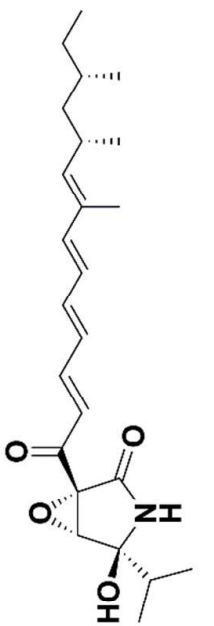

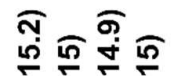

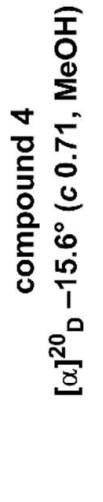

0

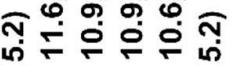

นิำ

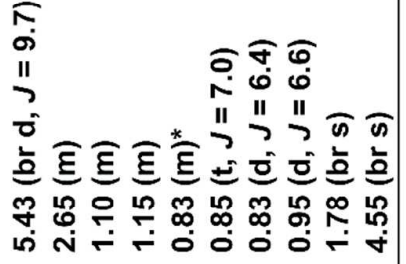

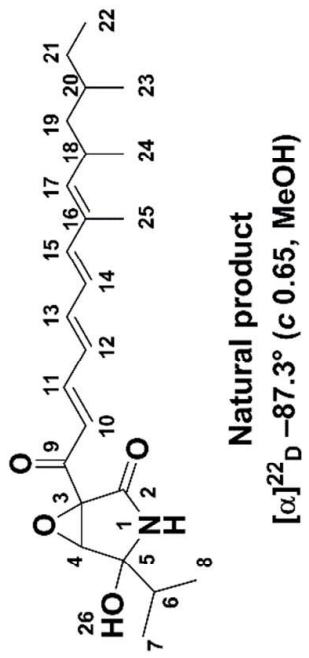

ヘ ำ

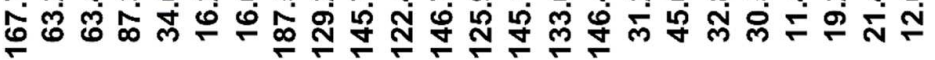

II $\rightarrow ? \geq ?$

흐흔 흠 흔 흘 흐

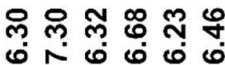

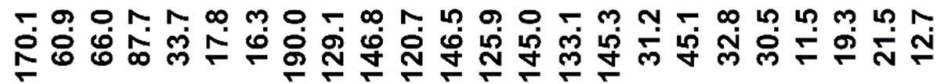

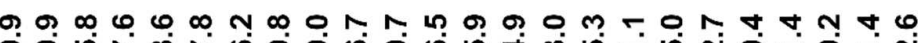

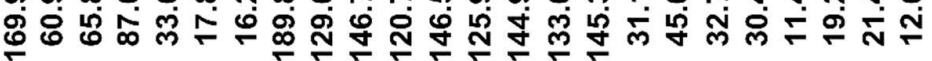

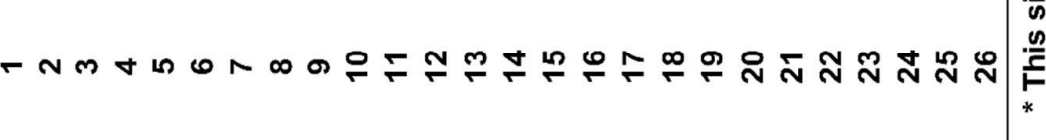

